\title{
EQUILIBRIA IN AN OVERLAPPING GENERATIONS MODEL WITH TRANSFER POLICIES AND EXOGENOUS GROWTH
}

\author{
JEAN-FRANÇOIS MERTENS ${ }^{\dagger}$ AND ANNA RUBINCHIK
}

\begin{abstract}
For an overlapping generations economy with varying life-cycle productivity, non-stationary endowments, continuous time starting at $-\infty$ (hence allowing for full anticipation), constant-returns-to-scale production and CES utility we fully characterise equilibria where output is higher than investment, which is strictly positive. Net assets (aggregate savings minus the value of the capital stock) are constant in any equilibrium, and, for balanced growth equilibria (BGE, defined for an economy with stationary endowments), net assets are non-zero only in the golden rule equilibrium, in accord with [12]. The number of BGE is finite. Their parity, however, depends on the life-cycle productivity, in particular, on the relation between the intertemporal elasticity of substitution, the minimal working age and the minimal tax age.
\end{abstract}

2000 Mathematics Subject Classification. 91B62

J.E.L. Classification numbers. D50.

Key words and phrases. Infinite Economies, Overlapping Generations, Exogenous Growth.

We would like to thank for its hospitality the Center for Rationality in Jerusalem, and for their comments participants of PET'08, SED'08, NBER General Equilibrium Conference at the University of Iowa, European General Equilibrium Conference at Warwick, European Econometric Society meetings at the University of Oslo, as well as the seminar participants in Paris.

This paper presents research results of the Belgian Program on Interuniversity Pôles of Attraction initiated by the Belgian State, Prime Minister's Office, Science Policy Programming. The scientific responsibility is assumed by the authors.

${ }^{\dagger}$ CORE, Université Catholique de Louvain; 34, Voie du Roman-Pays; B-1348 Louvain-la-Neuve; Belgique. E-mail: jfm@core.ucl.ac.be.

${ }^{\ddagger}$ Dept. of Economics; University of Haifa; Mount Carmel, Haifa, 31905; Israel.

E-mail: annarubinchik@gmail.com. 


\section{Contents}

1. Introduction 3

2. The setup 4

2.1. Individuals 4

2.2. Production 6

2.3. Equivalence Classes 8

2.4. Variants 8

2.5. Intensive variables 8

3. Characterisation of Equilibria 10

3.1. Some bounds stemming from feasibility 10

3.2. Individual demand 12

3.3. Optimality of production 13

3.4. Optimal investment 15

$\begin{array}{ll}\text { 3.5. First conclusions about equilibria } & 17\end{array}$

3.6. Aggregate demand 21

3.7. Avoiding the null equilbrium 22

4. The equilibrium equations 23

4.1. Implications of the equilibrium conditions 25

5. Balanced Growth Equilibria 26

5.1. Nonsensical (overinvestment) BGE 27

5.2. Golden rule equilibria 28

5.3. Graphs of BGE without transfers for $\mathbf{f}(\mathbf{k})=\mathbf{A} \mathbf{k}^{\alpha} \quad 28$

6. Gale's dichotomy 28

7. Finiteness and Parity of BGE 33

8. Conclusions 35

Appendix A. Aggregation of demand 36

Appendix B. The evaluation of profits 36

B.1. The "hot potato" example 36

$\begin{array}{lll}\text { B.2. The variation } & 37\end{array}$

B.3. Transactions and production sets 37

B.4. Transactions-based evaluation of profits 40

B.5. Integration by parts 41

B.6. Marking to market $\quad 42$

Appendix C. Analyticity properties of the BGE graph 43

Appendix D. A lemma of B. Weiss 44

$\begin{array}{ll}\text { References } & 44\end{array}$ 


\section{INTRODUCTION}

Consider the classical (Cass and Yaari [3]) 1-commodity, 1 type of consumer overlapping generations (OG) model with exogenous labour-saving technological growth, constant-returns-to-scale production function and time-separable CES utility. Time is the real line, individual life-span is [0,1], labour is supplied inelastically as in [3] and, in addition, life-cycle productivity is a non-negative function of age, which could be taken, for instance, as 1 in the first half of life, and 0 in the second, as in Samuelson's model [21]. We add arbitrary endowments, in particular, lump-sum transfers, as a template for general policies, and give an easy characterisation of competitive equilibria, that allows in [19] to justify (and provide an algorithm for) comparative statics with respect to policies, this being our main goal.

Recent variations of Cass and Yaari's model either provide a partial characterisation for very specific cases (output linear in capital, [6]; exchange economy with logarithmic preferences, [8]) or are obscure, mixing the description of the economy with equilibrium conditions.

The main contribution of this paper is the characterisation of competitive equilibria based on the classical general equilibrium approach: avoiding any ad-hoc restrictions on equilibrium variables, and deriving all properties of prices and quantities (finiteness, positivity, continuity, differentiability, etc.) from the equilibrium (i.e., optimisation) conditions. To the best of our knowledge we provide the first careful equilibrium analysis of this model, especially for non-stationary transfers (endowments). In particular, our price equation, lemma 10.a4-a5, shows that the standard form of no-arbitrage condition, taken for granted in the literature, is, in general, incorrect and holds only for equilibria where capital is never zero; otherwise, prices might not be smooth.

Our analysis further allows for irreversibility constraints, which can generate inequality of equilibrium prices (for consumption and output, for example).

We also establish finiteness of the number of balanced growth equilibria (BGE) (where the capital path is exponential), and that there is no 0-equilibrium: under mild conditions imposed on the production function, all BGE are interior.

Gale [12], who analysed a discrete-time pure-exchange OG economy, demonstrated it has two types of equilibria: balanced ones (where net savings equal the value of the capital stock) and the golden rule. ${ }^{1}$ Further, Diamond [9] showed the latter case is implied by some weak form of efficiency. Cass and Yaari's connection between positive net assets and (their notion of) Pareto efficiency is not applicable here, in particular, due to their reliance on logarithmic utility, non-Cobb-Douglas production, no transfers and constant life-time productivity. ${ }^{2}$ We show that Gale's insight is still true for "stationary" equilibria in the present setup: in a golden rule equilibrium (GRE) net savings almost always differ from the value of accumulated capital, while in any other balanced growth equilibrium (BGE) the two are equal; see section 6 for this dichotomy.

Not all classical results extend though: for instance, the number pure BGE (with zero net assets) is not necessarily odd, as was found in [15]. In particular, without any transfers, the number of pure BGE is even if the minimal working age exceeds the intertemporal elasticity parameter (by cor.18). This is then in accord with the non-existence of pure BGE claimed in [5] for a similar economy without production.

\footnotetext{
${ }^{1}$ Other work related to the dichotomy [2] is discussed in comment 32.

${ }^{2}$ We analyse the related efficiency issues in [17].
} 
While pure (lump-sum) transfers across contemporary consumers do not change GRE (cor. 15), their presence can affect even the parity of pure BGE (cor. 18). Positive aggregate transfer can lead to nonsensical (overinvestment) equilibria where aggregate consumption is strictly less than the aggregate endowment (see sect. 5.1).

In addition, under a mild restriction on technology, we find that there are no 'trivial' equilibria where capital is identically zero (see comm. 20). Ruling out equilibria where capital is sometimes zero, is far from trivial in this model (see cor. 12), although to the best of our knowledge in the previous literature such equilibria were ruled out by assumption (often made implicitly).

Absence of financial bubbles (i.e., "overpriced" capital stock) often taking the form of a transversality-like condition follows in the present set-up from profit maximisation of finitely-lived investment and production firms, see comment 19.

Finally (at least in case of Cobb-Douglas production), indeterminacy disappears in this model, as is shown in [18], which was one of the basic reasons to use $\mathbb{R}$ for time - as also partially confirmed since then in [8].

As to the model itself, it includes the following new features:

Individual productivity is an arbitrary positive function of age. Among others, it crucially affects the parity of BGE.

The distinction between production firms, transforming capital and labour instantaneously into 'output', merchandising firms, transforming output into either consumption or investment, and finitely-lived investment firms, whose technology is the capital accumulation equation: they buy the investment good from the merchandising firms and rent capital out to the production firms. Of course, any other setup (including, e.g., consumers being themselves the investment firms, as in [9]) with the same aggregate production possibilities is equivalent, and leads to the same equilibria. But the present distinction allows to focus on the main geometric aspects of the aggregate production set (of production and merchandising firms), and in particular to think properly about the irreversibility aspects for each type of transformations. If investment is a.e. strictly positive and strictly less than output (so the irreversibility constraints are not hit), we can speak of "interior equilibria"; those are independent of the irreversibility constraints, and there output, investment and consumption behave as a single good, with a single price, which is then also the price of capital.

Allowing individual consumption and prices (more generally, flows and their prices) to be any non-negative extended-real valued Lebesgue-measurable functions of time (or equivalence classes of such functions). With such definitions, equilibria are fully determined by the usual maximisation and market-clearing conditions only, without imposing them to lie in addition in some predetermined spaces. Since indirect utility can be infinite even if prices are positive and finite over the life-time, we use only very basic optimisation techniques to characterise individual demand (sect. 3.2). We extend accordingly (sect. 3.6, app. A) the classical approach $[1,7]$ to derive the demand correspondence.

Sect. 2 contains the model, sect. 4 the equilibrium equations (with preparations in sect. 3), sect. 5 characterisation of BGE, sect. 6 Gale's dichotomy, and finiteness and parity results for BGE are in sect. 7 . Conclusions follow.

\section{THE SETUP}

2.1. Individuals. $N_{x} d x \stackrel{\text { def }}{=} N_{0} e^{\nu x} d x\left(N_{0}>0\right)$ individuals get born in $[x, x+d x]$, $\forall x \in \mathbb{R}$, and live for 1 unit of time.

2.1.1. Individual preferences over consumption $c$, a $\overline{\mathbb{R}}_{+}$-valued Lebesgue-measurable function of age $s$, are represented as a discounted sum of homogeneous 
instantaneous "felicity" functions with intertemporal substitution $\sigma>0$ : letting $u(z)=\frac{z^{1-\frac{1}{\sigma}}}{1-\frac{1}{\sigma}}$ for $\sigma \neq 1$, extended by continuity to $[0,+\infty]$, individual utility equals

$$
U(c)=\int_{0}^{1} e^{-\beta s} u(c(s)) d s
$$

2.1.2. Labour. An individual can rent his time endowment (1 at each instant, $=100 \%$ ) partly out as labour; its efficiency varies with age $s$ according to a non-null integrable function $\zeta_{s} \geq 0$. Further, labour productivity grows with time at rate $\gamma$, as is classical in exogenous growth. So, total productive labour available at $t$ equals:

$$
L_{t}=N_{0} e^{\gamma t} \int_{t-1}^{t} \zeta_{t-x} e^{\nu x} d x=N_{0} e^{(\gamma+\nu) t} \int_{0}^{1} \zeta_{s} e^{-\nu s} d s
$$

His time sells for $\int_{0}^{1} w_{x+s} \zeta_{s} d s$, where $x$ is his birth-date and $w_{t}$ is the (per unitefficiency) wage rate at time $t$, an $\overline{\mathbb{R}}$-valued Lebesgue measurable function of time.

2.1.3. Endowments. His initial endowment of consumption goods is $\omega_{x, s}$ at age $s$. $\omega$ is locally integrable. ${ }^{3}$ Its sign is not restricted, in order to represent a.o. arbitrary transfer policies. Similarly, $\hat{c}_{x, s}$ denotes a point in his consumption set.

Denote the price of consumption goods by $p^{C}$, an $\overline{\mathbb{R}}_{+}$-valued Lebesgue-measurable function of time. The cost of any consumption bundle $c, \int_{0}^{1} p_{x+s}^{C} c(s) d s$, is then welldefined. ${ }^{4}$ Shares in profits are null (constant returns to scale production), so the individual's lifetime wealth is the value of his endowment (of consumption goods and of leisure), $M_{x} \stackrel{\text { def }}{=} \int_{0}^{1}\left(p_{x+s}^{C} \omega_{x, s}+w_{x+s} \zeta_{s}\right) d s$, provided that integral is well-defined. ${ }^{5}$

2.1.4. Consumer optimisation. Allowing agents to buy commodities outside their lifetime will not change the the aggregates in the equilibria characterised here. ${ }^{6}$

By 2.1.3, the budget set (paths of consumption and leisure) is now well-defined whenever $M_{x}$ is, and hence so is utility-maximisation over the budget set. Thus, the criterion for consumer optimisation is that almost all agents with well-defined $M_{x}$ maximise their utility over their budget set.

In the equilibria we will characterise, $M_{x}$ will be well-defined a.e. ${ }^{7}$

Comment 1. One might argue whether the above definition is correct in case where both prices and wealth are infinite; but infinite prices will not occur in any equilibrium, for other reasons, ${ }^{5}$ so we might as well have left individual demand to be the whole positive orthant in that case, without affecting the results; the above was chosen just for greater simplicity in the phrasing. Similarly, lemma 4 below will show that, with a.e. finite prices, the maximal utility is always achieved; so the above requirement of exact maximisation implies no further hidden restrictions: the definition of equilibrium is the same as if consumer optimisation applied only when further the consumer does have a maximal element in his budget set.

\footnotetext{
${ }^{3} \mathrm{~A}$ function is locally integrable on a topological space if every point has a measurable neighbourhood where the function is integrable. $L_{1}^{\text {loc }}$ is the space of such functions.

${ }^{4} \mathrm{By}$ the usual convention in measure theory, define any product of prices and quantities as 0 in case of a product $0 \times \infty$ or $\infty \times 0$. This allows to think of either prices or quantities as measures.

${ }^{5}$ Def. 1 and lemma 5 .a imply $p^{C}$ is a.e. finite, and by local integrability $\omega$ is so too: the integrand is a.e. well-defined. Thus, we mean here, either its positive or its negative part is summable.

${ }^{6} \mathrm{Cf}$. the proof lemma 11. Elsewhere we assume, for simplicity, that individual consumption outside one's life-time is nil.

${ }^{7}$ Of course, the above criterion (for consumer optimisation) makes sense only if it implies that in equilibrium $M_{x}$ is well-defined a.e. But requiring the latter as part of the definition of equilibrium would be quite unsatisfactory: it would mean a restriction on the price-system, instead of on primitives, and one that has no conceptual meaning whatsoever: it depends completely on the integration theory used. Unreasonable solutions (price-systems) should be excluded by clear equilibrium arguments, not by fiat.
} 
2.1.5. Aggregation of the demand correspondence is discussed in sect. 3.6.

2.2. Production. Firms have finite lives, for profits to be well-defined.

2.2.1. The instantaneous production set is a subset of $\mathbb{R}^{5}$ describing feasible transformations of contracted productive labour $L_{t}$, capital $K_{t}$, investment $I_{t}$, consumption $C_{t}$ and an intermediate good $Y_{t}$ called 'output', produced using a constant returns to scale technology with $Y_{t}=F\left(K_{t}, L_{t}\right), F: \mathbb{R}_{+}^{2} \rightarrow \mathbb{R}_{+}$concave, continuous, positively homogeneous of degree 1 , and differentiable on $\mathbb{R}_{++}^{2} \cdot f(x) \stackrel{\text { def }}{=} F(x, 1)$.

The production set is any closed convex cone with free-disposal, containing the graph of the production function and the activities of transforming output into consumption and investment, and contained in the closed convex cone spanned by the production function, free-disposal, and 2-way transformations of output into consumption and investment. This allows thus for any possible form of 'irreversibility'. ${ }^{8}$

Manufacturing produces undifferentiated output $Y_{t}$, using the above technology, from labour $L_{t}$, bought from individuals at price $w_{t}$, and capital $K_{t}$, rented from investment firms at rate $r_{t}$.

Merchandising transforms $Y_{t}$ (with price $p_{t}^{Y}$ ) one-to-one into either the consumption good $C_{t}$ or the investment good $I_{t}$. This transformation may or may not be partially reversible depending on the instantaneous production set. $C_{t}$ is sold at price $p_{t}^{C}$ to individuals and $I_{t}$ at price $p_{t}^{I}$ to investment firms.

Comment 2. This distinction is just to give a convenient informal language; one can't distinguish 2 types of firm: it is not true that any instantaneous production cone as above decomposes into the sum of 2 closed convex cones, one in $(Y, L, K)$ space corresponding to manufacturing and one in $(C, I, Y)$-space for merchandising.

Note that if $p_{t}^{Y}=\infty$, profits of any production plan with positive output are either infinite or, if also $w_{t}$ or $r_{t}$ are $\infty$, undefined. This is incompatible with any equilibrium concept, so we exclude it formally:

Definition 1. $p_{t}^{Y}<\infty$ a.e.

2.2.2. Capital $K_{t}$ accumulates as $K_{t}^{\prime}=I_{t}-\delta K_{t}$; to make this precise, one would need to spell out the exact differential equation theory used and the exact properties required of the functions $K_{t}$ and $I_{t}$. To this effect we reinterpret this in integral form; ${ }^{9}$ formally: $K_{t}=e^{-\delta\left(t-t_{0}\right)} K_{t_{0}}+\int_{t_{0}}^{t} e^{-\delta(t-s)} I_{s} d s$ as a wide Denjoy integral. ${ }^{10}$

Cor. 2 will show that the above integral is in fact a plain Lebesgue integral. ${ }^{11,12}$

As any differential equation, the capital accumulation equation needs an initial condition, which is part of the description of what is feasible, of the technology: ${ }^{13}$

Assumption 1 (Initial Condition). For any feasible $K, e^{\left(\delta-f_{\infty}^{\prime}\right) t} K_{t}$ converges exponentially to 0 at $-\infty$, where $f_{\infty}^{\prime} \stackrel{\text { def }}{=} \lim _{x \rightarrow \infty} \frac{f(x)}{x}$.

\footnotetext{
${ }^{8} Y$ being a purely intermediate good could as well be netted out. One gets then a closed convex cone in $\mathbb{R}^{4}$, contained in $\{C+I \leq F(K, L)\}$ and containing $\{\max (C, I, C+I) \leq F(K, L)\}$.

${ }^{9}$ The integral form yields the possibility to use arbitrary integrable $I_{t}$ rather than exact derivatives, which is crucial e.g. in [18]. Its interpretation is as direct as that of the differential equation: $K_{t}$ is what remains after depreciation from $K_{t_{0}}$ and the intervening investments.

${ }^{10}$ For the right interpretation of the capital-accumulation equation, one needs, as argued in [20], to use Denjoy rather than Lebesgue integration, in order to include the classical solutions of this differential equation - and then one may as well use the most encompassing integration theory: the integral above $i s$ meant as a wide Denjoy integral [e.g., 4, p. 27].

${ }^{11}$ Its proof does not use any specific properties of the wide Denjoy integral: it applies to any monotone linear extension of the Lebesgue integral to some vector space of Lebesgue-measurable functions for which primitives are locally bounded (or even just: in $L_{1}^{\text {loc }}$ ).

${ }^{12}$ And thus the corresponding differential equation theory can be taken as Caratheodory's.

${ }^{13}$ Cf. app. B.3 for a more formal treatment of the aggregate production set, in this respect too.
} 
The word "exponentially" can be dropped here ${ }^{14}$ iff $\int_{1}^{\infty} \frac{f(x)-x f_{\infty}^{\prime}}{x^{2}} d x<\infty .{ }^{15,16}$

2.2.3. Investment Firms are finitely lived, for their profits to be well-defined. During its lifetime $\left[t_{0}^{f}, t_{1}^{f}\right]$, firm $f$ buys and sells capital stock at price $p_{t}$ and an investment flow $I_{t}^{f}$ at price $p_{t}^{I}$, and get rents $r_{t} K_{t}^{f}$ from capital services rented out to manufacturing firms. Its technology is the capital accumulation equation, and its capital stock $K_{t}^{f}$ must be non-negative at all times.

We assume, as in [18, sect.3.1.2], that investment firms can disvest as well as invest: all restrictions on disvestment are written in the instantaneous production set, i.e., if disvestment is not possible for some capital good, any sale of that investment good by an investment firm can only be to another investment firm, and can be interpreted as being the transfer of the corresponding capital.

We allow for a complete measure space $(F, \mathcal{F}, \mu)$ of investment firms, a.o. to include the case where the consumers would do all the investing, and assume:

Assumption 2. (a) $t_{0}^{f}$ and $t_{1}^{f}$ are measurable, $t_{0}^{f}<t_{1}^{f}$ a.e.;

(b) $\forall t \exists \varepsilon: \mu\left\{f \mid t_{0}^{f}<t+\varepsilon, t_{1}^{f}>t-\varepsilon\right\}<\infty$;

(c) $\forall t, \mu\left(G_{t_{-}}\right)>0, \mu\left(G_{t_{+}}\right)>0$, with $G_{t_{-}}=\left\{f \mid t_{0}^{f}<t \leq t_{1}^{f}\right\}, G_{t_{+}}=\left\{f \mid t_{0}^{f} \leq t<t_{1}^{f}\right\}$;

The corresponding production plans should satisfy the following — the need for assumption 3.d is illustrated in app. B.1:

Assumption 3. (a) $I_{t}^{f}$ and $K_{t}^{f}$ are locally in $t$ jointly integrable in $(t, f)$, and $K_{t}^{f}$ is $\mu \times \nu$-measurable for any bounded measure $\nu$ on $\mathbb{R}$;

(b) $K_{t}^{f} \geq 0$; and $\forall t \notin\left[t_{0}^{f}, t_{1}^{f}\right], K_{t}^{f}=I_{t}^{f}=0$;

(c) $\int I_{t}^{f} \mu(d f)=I_{t}$ a.e.; $\int K_{t}^{f} \mu(d f)=K_{t} \forall t$;

(d) $\forall t, K_{t_{-}}^{f}$ and $K_{t_{+}}^{f}$ exist a.e., and $\lim \inf K_{t-\varepsilon}>0 \Rightarrow \int K_{t_{-}}^{f}>0, \lim \inf K_{t+\varepsilon}>$ $0 \Rightarrow \int K_{t_{+}}^{f}>0$.

Remark 3. For feasible allocations, the assumptions in sub d mean simply $K_{t}>0$. But we need the above phrasing for the discussion (prop. 2 in app. B.3).

Remark 4. Ass. 3.a means $\forall t \exists \varepsilon: I_{t}^{f}, K_{t}^{f}$ are integrable on $[t-\varepsilon, t+\varepsilon] \times F$. It, and 2.a,3.b, imply (Fubini) that $\left\{f \mid I_{t}^{f}\right.$ or $\left.K_{t}^{f} \notin L_{1}\right\}$ is a null set-which we will ignore.

Remark 5. Prop. 3 in app. B.3 shows assumptions 2 and 3 imply no restriction on the aggregates. Further, given the equilibrium conditions we finally obtain, clearly no firm $f$ could profitably deviate even if it were allowed Denjoy-integrable $I_{t}^{f}{ }^{17}$

Comment 6 . Ass. 3 seems out of place: normally one defines production sets $Z_{f}$ for each firm $f$, and the aggregate production set $Z$ is just the integral of the correspondence $f \mapsto Z_{f}$-i.e., the allowed production plans are the integrable selections of this correspondence. And, e.g., part 3.d does not look at all like an integrability requirement. We show nevertheless in app. B.3 that such a standard approach is possible, and that ass. 3 is then an implication, and further that the whole paper is consistent as well with this more standard approach (a.o., no implied aggregate restrictions).

\footnotetext{
${ }^{14}$ Getting thus the initial condition of [20] in its weakest form, as in App. C loc. cit. It would indeed have been unsatisfactory to require an exponentially fast convergence at $-\infty$ when one of the purposes of the dependent paper [18] is to establish such a stability property.

${ }^{15}$ This extends the "Strong No-Rabbit" condition of [20, App. C] to the present case, and covers all "classical" cases like Cobb-Douglas or CES.

${ }^{16}$ Indeed, prop. 1.a implies that any feasible $k$ is then bounded, which implies the exponential convergence. The 'only if' aspect results from a careful reading of that proof.

${ }^{17}$ Indeed, the proof of sufficiency in lemma 7 remains applicable, and the profits formula it relies on, from app. B.6, allows for arbitrary Denjoy primitives as $K$-no need for bounded variation.
} 
2.2.4. Investment profits. The evaluation of profits of the investment firms is discussed in app. B.4, B.6 (and B.1 for motivation). In particular, as mentioned in the introduction, we refuse to impose additional restrictions on endogenous variables like prices, that would stem from assuming them to belong to some 'spaces', instead of proving that equilibrium forces them to be there. In particular, prices of flows $\left(C_{t}, I_{t}\right)$ are naturally thought as non-negative $\overline{\mathbb{R}}$-valued Lebesgue-measurable functions of time, but for the price of a stock, like capital, even Lebesgue-measurability has no reason to be there. Thus the possible prices a priori cannot belong to the space of continuous linear functionals on the topological vector space containing the production sets, and we will have to resort to integration theory to define profits. There will even be some combinations of a price system and a feasible production bundle for which profits cannot be defined; so we will just assume that, whenever profits are well-defined, they are non-positive, and prove that, in the final characterisation thus obtained, profits are well-defined and $\leq 0$ over all production sets $Z_{f}$.

2.3. Equivalence Classes. Observe that for any individual consumption bundle $c$ any equivalent function (coinciding with $c$ a.e.) has the same utility and the same budget, so we will think of it as an equivalence class of $\overline{\mathbb{R}}_{+}$-valued measurable functions. The same applies to all flows, $Y_{t}, I_{t}, C_{t}$, labour- and capital-services, and to their prices $p^{Y}, p^{I}, p^{C}, w$, and $r$. On the other hand capital is a stock, so it and its price $p_{t}$ are defined pointwise, and no measurability restriction has a reason to apply.

2.4. Variants. The general model above allows for irreversibility, and, with it, is a particular case of the model described in [20]. So it is this that we need to provide in [16] the "proof of non-vacuity" for [20].

The - classic - variant is where consumption and investment are freely transformable into each other, thus effectively defining a 1 good model; we will refer to this as the basic model, which will be used to establish results for the general model.

2.5. Intensive variables. Let $\mathfrak{E}$ denote the economy described above.

2.5.1. Further parameters for $\mathfrak{E} . \varphi_{s}=\frac{e^{-\nu s} \zeta_{s}}{\int_{0}^{1} e^{-\nu u} \zeta_{u} d u}, \eta=(\gamma+\nu)(1-\sigma)+\beta \sigma, E_{t, s}=$ $\frac{N_{t-s} \omega_{t-s, s}}{L_{t}},{ }^{18} \Omega_{t}=\int_{0}^{1} E_{t, s} d s$.

2.5.2. Further variables for $\mathfrak{E}$. Whatever the variables $K_{t}, Y_{t}$, etc. stand for in the context of a particular statement (referring e.g. to some specific feasible path, or to demand or supply in a specific equilibrium candidate), we will use the shorthands (with $N_{x}$ and $L_{t}$ from sect. 2.1 and 2.1.2) $k_{t}=\frac{K_{t}}{L_{t}}, y_{t}=\frac{Y_{t}}{L_{t}}, i_{t}=\frac{I_{t}}{L_{t}}, c_{t}=\frac{C_{t}}{L_{t}}$, $c_{t, s}^{\odot}=\frac{N_{t-s} \hat{c}_{t-s, s}}{L_{t}},{ }^{18}\left(\right.$ so $\left.c_{t}=\int_{0}^{1} c_{t, s}^{\odot} d s\right)$.

2.5.3. The reduced economy. Define now a new economy $\mathfrak{E}^{\prime}$ as the original one $\mathfrak{E}$ with the following parameters: $\gamma=0, \nu=0, \delta=R, \zeta=\varphi, \sigma=\sigma, \beta=\eta / \sigma$, $\omega_{x, s}=E_{x+s, s}, N_{0}=1\left(\right.$ so $\left.L_{t}=1\right)$.

2.5.4. Isomorphism of $\mathfrak{E}$ and $\mathfrak{E}^{\prime}$. First we give a general definition of isomorphism of economies, ${ }^{19}$ in case of constant returns to scale production, so profit shares can be ignored. The general case is sketched in fn. 20 .

\footnotetext{
${ }^{18} E_{t, s}$ (resp., $c_{t, s}^{\odot}$ ) is the normalised [per unit of productive labour at time $t$ ] aggregate endowment (resp. consumption) at time $t$ of individuals of age $s$.

${ }^{19}$ Isomorphism here is in a sense somewhat generalised from that of [20] because the measure on agents is changed in a non-constant way, i.e., the concept there is extended by the following 2 operations: (a) change the measure of an agent, re-scaling his endowment and preferences accordingly, and (b) replace 2 identical agents by a single agent having as mass the sum of their masses ("2 economies with the same distribution of characteristics are isomorphic").
} 
Definition 2. Take two economies $\mathfrak{E}_{i}(i \in\{1,2\})$ with the corresponding measure spaces of agents (denoted by $\left(A_{i}, \mathcal{A}_{i}, \alpha_{i}\right)$ ) and firms, production and consumption sets, and preferences. An isomorphism between $\mathfrak{E}_{1}$ and $\mathfrak{E}_{2}$ is any composition of the following 3 elementary isomorphisms and/or their inverses: ${ }^{20}$

(a) Rescaling agents: the map is the identity, except that $\alpha_{1}$ and $\alpha_{2}$ are mutually absolutely continuous, s.t. if $h=\frac{d \alpha_{1}}{d \alpha_{2}}$, then $\omega_{2}(a)=h(a) \omega_{1}(a)$ and $a$ prefers $x$ to $y$ in $\mathfrak{E}_{1}$ iff he prefers $h(a) x$ to $h(a) y$ in $\mathfrak{E}_{2}$.

(b) Mapping agents: a measurable map of agents $g: A_{1} \rightarrow A_{2}$ which is measurepreserving: for any coalition $A \in \mathcal{A}_{2}, \alpha_{2}(A)=\alpha_{1}\left(g^{-1}(A)\right)$. Further, $\forall a \in$ $A_{2}$, all agents in $g^{-1}(a)$ have the same preferences and endowments. ${ }^{21}$

(c) Commodity map: a linear map of commodity spaces, mapping production sets, consumption sets, endowments and preferences of $\mathfrak{E}_{1}$ onto those of $\mathfrak{E}_{2}$.

Remark 7 . When consumption sets are the positive orthant, a map as sub c reduces to a bijection between names of commodities in both economies together with a rescaling of each commodity (change of units).

Lemma 1. $\mathfrak{E}$ and $\mathfrak{E}^{\prime}$ are isomorphic, using the following sequence of elementary isomorphisms: (a) Map all agents born at $x$ to a single agent born at $x$. (b) Rescale the mass $N_{0} e^{\nu x} d x$ of agents born in $[x, x+d x]$ to $d x$. (c) Map productive labour and commodities dated $t\left(I_{t}, C_{t}, K_{t} \ldots\right)$ to themselves, dividing quantities by $L_{t}$.

Proof. The map is by definition an isomorphism, all agents born at $x$ being identical; so one has to show that its image is $\mathfrak{E}^{\prime}$. The population measure is indeed Lebesgue measure, by definition. Consumption sets remain the same, being the positive orthant, so unaffected by rescalings. Similarly, the instantaneous production set remains the same, being a cone, so unaffected under rescaling by the constant $L_{t}$.

A commodity bundle $\hat{c}_{x}$ for an agent born at $x$ becomes $N_{0} e^{\nu x} \hat{c}_{x}$ by the rescaling, then, by the commodity map, $\hat{c}_{x, s}^{\prime}=N_{0} e^{\nu x} \hat{c}_{x, s} / L_{x+s}$ which is $c_{x+s, s}^{\odot}$, and $\omega_{x, s}$ becomes $E_{x+s, s}$. By the homogeneity of degree $1-\frac{1}{\sigma}$ of $u$ and the formula for $L_{t}, \int_{0}^{1} e^{-\frac{\eta}{\sigma} s} u\left(\hat{c}_{x, s}^{\prime}\right) d s=\left(\int_{0}^{1} \zeta_{s} e^{-\nu s} d s\right)^{\frac{1}{\sigma}-1} \int_{0}^{1} e^{-\frac{\eta}{\sigma} s} e^{-(\gamma x+(\gamma+\nu) s)\left(1-\frac{1}{\sigma}\right)} u\left(\hat{c}_{x, s}\right) d s$; $=\left[e^{\gamma x} \int_{0}^{1} \zeta_{s} e^{-\nu s} d s\right]^{\frac{1}{\sigma}-1} \int_{0}^{1} e^{-\beta s} u\left(\hat{c}_{x . s}\right) d s$ by the formula for $\eta$, so utilities are preserved up to a multiplicative constant.

As to labour, an input of $l_{x, s}$ units of time in $\mathfrak{E}$ becomes $N_{0} e^{\nu x} l_{x, s}$ units of time after rescaling mass, i.e., $N_{0} e^{\nu x} e^{\gamma(x+s)} l_{x, s} \zeta_{s}$ productive labour, so, after the commodity map (division by $L_{t}$ ), $e^{-\nu s} l_{x, s} \zeta_{s} / \int_{0}^{1} e^{-\nu u} d u=l_{x, s} \varphi_{s}$, which is indeed the productive labour corresponding to $l_{x, s}$ in $\mathfrak{E}^{\prime}$, by our above formula.

Remains the technology of investment firms. For the capital accumulation equation, this is obvious (especially in the formal general form of sect. 2.2.2, $e^{\delta t} K_{t}-$ $e^{\delta t_{0}} K_{t_{0}}=\int_{t_{0}}^{t} e^{\delta s} I_{s} d s$ ), and for the initial condition (in both forms) as well.

\footnotetext{
${ }^{20}$ In general (absence of constant returns to scale), one needs also the following (we continue to assume the standard convexity assumptions, and assume that profit shares are described by a transition probability $\pi$ from the measure space of firms to that of agents such that $\pi(A \mid f)$ is negligible for every null set $A$ ): (1) For mapping agents (b), require further that, $\forall B \in \mathcal{A}_{1}$, $\forall S \in \mathcal{F}, \int_{S} \pi_{1}(B \mid f) \mu(d f)=\int \mathrm{P}\left(B \mid g^{-1}\left(\mathcal{A}_{2}\right)\right) \int_{S} \pi_{2}\left(d a_{2} \mid f\right) \mu(d f)$ (collapsed agents have the same profit shares). (2) A rescaling as sub (a) for the firms. (3) As for (b), collapsing firms with identical production sets into a single firm, in a mass-preserving way, and with as profit shares the average of the original profit shares. (4) Collapsing firms with identical profit distributions into a single firm, with as production set the integral of those of the constituent firms.

A.o., the above suffice to make any economy isomorphic to the corresponding private production economy (which is, for our model, a classical interpretation for the investment sector). One might then of course want to add Rader's trick, to reduce those to pure exchange economies with 0 endowments, and then possibly, to get rid of irrelevant parts of the consumption sets, that 2 agents in such a pure exchange economy with identical excess demand functions can be identified.

${ }^{21}$ One could allow for different endowments in case of homogeneous preferences.
} 
2.5.5. Variables for $\mathfrak{E}^{\prime}$. The variables $K_{t}, Y_{t}, I_{t}, C_{t}, \hat{c}_{x, s}$ for $\mathfrak{E}^{\prime}$ are thus $k_{t}, y_{t}, i_{t}, c_{t}$, $c_{x+s, s}^{\odot}$; and, since $L_{t}=1$ in $\mathfrak{E}^{\prime}$, the "further variables" for $\mathfrak{E}^{\prime}$ are the same.

\section{Characterisation of Equilibria}

Notation 3.1. $\lambda$ denotes Lebesgue measure on $\mathbb{R}$. $\Phi(x)=\frac{e^{x}-1}{x}$. For $h: \mathbb{R} \rightarrow \mathbb{R}$, $\|h\|_{\infty, 1}=\sup _{x} \int_{x-1}^{x}|h(t)| d t$, and $\|E\|_{\infty, 1}=\sup _{x} \int_{x-1}^{x} \int\left|E_{t, s}\right| d s d t . R=\gamma+\nu+\delta$.

Assumption 4. $\|E\|_{\infty, 1}<\infty$.

$$
f_{\infty}^{\prime}<R,{ }^{22} \text { and } \exists x: f(x)>R x \text {; i.e., } F(1,0)<R<F(1, \infty) .
$$

\subsection{Some bounds stemming from feasibility.}

Lemma 2. For a concave continuous function $\phi: \mathbb{R}_{+} \rightarrow \mathbb{R}$, with $\phi(0) \geq 0, \phi_{\infty}^{\prime} \in \mathbb{R}$, and $\sup _{x} \phi(x)>0$, let $\bar{\kappa} \stackrel{\text { def }}{=} \sup \{x \mid \phi(x) \geq 0\}$. The solutions (Caratheodory or classical) of the differential equation $k_{t}^{\prime}=\phi\left(k_{t}\right)$ are of one of the following 4 types: (a) $k_{t}$ for $t \in \mathbb{R}$ is strictly convex and decreases from $\infty$ to $\bar{\kappa}$, (b) $k_{t}=x \forall t$, for $x \in\{0, \bar{\kappa}\} \cap \mathbb{R}$, (c) If $\phi(0)>0, k$ has domain $\left[T, \infty\left[\right.\right.$ with $k_{T}=0$ and increases strictly to $\bar{\kappa}$, (d) If $\phi(0)=0, k_{t}$ has domain $\mathbb{R}$, and is 0 until some $T$, then increases strictly from 0 to $\bar{\kappa}$. $T$ is finite iff $1 / \phi$ is locally integrable at 0 , else $T=-\infty$.

Further, in the non-constant parts of solutions, $k$ is the inverse of a primitive of $\frac{1}{\phi}$; and within each type the solutions differ only by a time-shift.

Comment 8. In particular, uniqueness fails iff $\phi(0)=0$ and $1 / \phi$ is locally integrable at 0 , with 0 as initial value (e.g., the Cobb-Douglas case).

Proof. $\phi$ is locally Lipschitz except possibly at 0 , so the standard uniqueness theorem applies there. In particular, if $\bar{\kappa} \in \mathbb{R}, k_{t}=\bar{\kappa}$ is such a unique solution, so any other solution lies everywhere either above it or below. The former are clearly decreasing and strictly convex [since $\left.k_{t}^{\prime \prime}=\phi^{\prime}\left(k_{t}\right) \phi\left(k_{t}\right)\right]$; and since $\phi(x) \geq x \phi_{\infty}^{\prime}$, they cannot blow up to $+\infty$ before $t$ gets to $-\infty$ : they are defined on $\mathbb{R}$, and decrease from $+\infty$ to $\bar{\kappa}$. It is then easily seen that their convergence to $+\infty$ at $-\infty$ and to $\bar{\kappa}$ at $+\infty$ is exponential.

The others are strictly increasing when $>0$, from 0 to $\bar{\kappa}$, converging again to $\bar{\kappa}$ at $+\infty$, exponentially if $\phi_{\infty}^{\prime} \neq 0$. Remains thus to check the case $k_{t}=0$.

If $\phi(0)>0, k_{t} \equiv 0$ is not a solution, so let $k_{t_{1}}>0$. Since $k_{t}<\bar{\kappa} \forall t$ and is increasing, its slope before $t_{1}$ is bounded away from 0 , so any solution hits 0 at a finite time $t_{0}$. And clearly there is no continuation (even Caratheodory) for $t<t_{0}$ : solutions are defined on $\left[t_{0}, \infty[\right.$, starting with the value 0 and a positive derivative.

If $\phi(0)=0, k_{t} \equiv 0$ is a solution. And since any solution $k_{t}$ is increasing and unique as soon as positive, $\exists t_{0} \in \overline{\mathbb{R}}: k_{t}=0$ iff $t \leq t_{0}$. Then, for $t>t_{0}$, the differential equation is equivalent to $\frac{d k}{\phi(k)}=d t$. Assume $t_{0}<\infty$, and fix $\left.t_{1} \in\right] t_{0}, \infty[$. Let $h(x)=\int_{k_{t_{1}}}^{x} \frac{1}{\phi(z)} d z$, so $t=t_{1}+h\left(k_{t}\right)$. As seen above, $k_{t}$ remains after $t_{0}$ in $\{k \mid \phi(k)>0\}$, and there $h(x)$ is well defined, strictly increasing and continuous, and $h(x) \underset{x \rightarrow \kappa}{\longrightarrow} \infty$, so the inverse function $h^{-1}$ is well-defined from $[h(0), \infty[$ to $[0, \bar{\kappa}[$. And when $t \searrow t_{0} k_{t} \searrow 0$, so $h(0)=t_{0}-t_{1}$. Thus $k_{t}=h^{-1}\left(t-t_{1}\right) \forall t>t_{0}$.

$h(0)$ and thus $t_{0}$ is finite iff $\frac{1}{\phi}$ is locally integrable at 0 , and then, as $t \searrow t_{0}$, $k_{t} \searrow 0$ and so $k_{t}^{\prime}=\phi\left(k_{t}\right) \searrow 0$ : letting $k_{t}=0 \forall t \leq t_{0}$ defines the solution on $\mathbb{R}$, and it is $C^{1}$ even at $t_{0}$. In the other case also, the solutions are defined on $\mathbb{R}$, but then either strictly increasing or identically 0 .

Corollary 1. If $\phi(0)=0$ and $\phi^{\prime}(0)=\infty$, and $k_{t}$ solves the differential equation in lemma 2 with $k_{t_{0}}=0$, then for all $\varepsilon>0 \phi^{\prime}\left(k_{t}\right)$ is not integrable on $\left[t_{0}, t_{0}+\varepsilon\right]$.

\footnotetext{
${ }^{22}$ This is the sharp form of the 'No-Rabbit' assumption in [20].
} 
Proof. If $k_{t}>0 \forall t>t_{0}$, then, by lemma $2, \frac{1}{\phi(\cdot)}$ is locally integrable at 0 , and with $h(x)=\int_{0}^{x} \frac{1}{\phi(z)} d z$ as in lemma $2, k_{t}=h^{-1}\left(t-t_{0}\right) \forall t \geq t_{0}$, so $\phi^{\prime}\left(k_{t}\right)=\phi^{\prime}\left(h^{-1}(t-\right.$ $\left.t_{0}\right)$ ). Substituting $t$ by $t_{0}+h(k)$ in $\int_{t_{0}}^{t_{0}+\varepsilon} \phi^{\prime}\left(h^{-1}\left(t-t_{0}\right)\right) d t$ we get $\int_{0}^{k_{\varepsilon}} \frac{\phi^{\prime}(k)}{\phi(k)} d k$, then, substituting $\phi(k)$ by $z, \int_{\phi(0)}^{\phi\left(k_{\varepsilon}\right)} \frac{1}{z} d z,=\infty$ as $\phi(0)=0$.

Else, by lemma $2, \exists \varepsilon>0: k_{t}=0 \forall t \in\left[t_{0}, t_{0}+\varepsilon\right]$, so $\phi^{\prime}\left(k_{t}\right)=\infty$ on $[0, \varepsilon]$.

Proposition 1. For any feasible allocation:

(a) $\exists A, B: \forall \omega, \kappa \stackrel{\text { def }}{=} \sup _{t} k_{t} \leq A\|\Omega\|_{\infty, 1}+B$. And $\sup _{t} y_{t} \leq f(\kappa)$.

(b) $\|i\|_{\infty, 1} \leq(1+R) \kappa+2\left(f(\kappa)+\|\Omega\|_{\infty, 1}\right) .^{23}$

(c) Let $\bar{c}_{x, s}$ be the consumption at age $s$ of an agent born at time $x . v_{x} \stackrel{\text { def }}{=}$ $e^{-\gamma x}\left(\int_{0}^{1} \bar{c}_{x, s}^{1-\frac{1}{\sigma}} d s\right)^{\frac{\sigma}{\sigma-1}}$ and $c_{t}$ are locally integrable; specifically, $\|c\|_{\infty, 1} \leq$ $\|\Omega\|_{\infty, 1}+f(\kappa)+\|i\|_{\infty, 1}$ and $\|v\|_{\infty, 1} \leq 2 e^{|\gamma|+|\nu|}\|c\|_{\infty, 1} \int_{0}^{1} \zeta_{s} e^{-\nu s} d s$.

Remark 9. Besides the bound for $v$ sub c, b is basic: via cor. 2 below, it implies a bound for all feasible $\|k\|_{\infty}$ and hence $\|y\|_{\infty}$ as sub a, and thus one for $c$ as sub c.

Proof. a: Let $\psi(x)=f(x)-R x, \phi=\psi+P$ with $P=\|\Omega\|_{\infty, 1}$, and $\kappa_{P}$ be the positive root of $\phi(x)=0$. By concavity of $f$ and ass. $4, \exists a, b>0: \psi(x) \leq-a x+b$, so $\kappa_{P} \leq \frac{b+P}{a}$. Suffices thus to prove that for any feasible path $k_{t}, k_{t} \leq \kappa_{P}+P$. Replace $\Omega_{t}$ by $\left|\Omega_{t}\right|$, so $\Omega_{t} \geq 0$. If else e.g. $k_{0}>\kappa_{P}+P$, then $k_{t}>\kappa_{P}$ on $[-1,0]$ : indeed, even if all aggregate transfers $\Omega_{t}$ for $t \in[-1,0]$ are pushed to time 0 , then $k_{0-}>\kappa_{P}, \geq \kappa_{0}$, and thus $k_{t}$ must be decreasing on [-1,0[, since even if all agents work full-time and consume nothing it decreases by lemma 2 . Thus $k_{t}>\kappa_{P}$ in this case. And if part of the transfers occur earlier in $[-1,0[$, the conclusion holds a fortiori, since we are in a region where (additions to) capital cannot be maintained.

Any time-shift of the feasible allocation is still feasible. Do thus a convolution (an average of time-shifts) of $\Omega_{t}, i_{t}$ and $k_{t}$ with a uniform distribution on [0,1]: the new $\Omega_{t}, i_{t}$ and $k_{t}$ are still feasible, by linearity, and concavity of $f$, and now $\|\Omega\|_{\infty} \leq P$ and $k_{0}>\kappa_{P}$. We can then further increase $\Omega$ s.t. $\Omega_{t}=P$ everywhere.

Let (for $t \leq 0$ ) $\varkappa_{t}$ be the minimal amount of capital needed to reach $k_{0}$ at time 0 : it is when agents work full-time and consume nothing, so it is (uniquely, cf. lemma 2) given by the differential equation $\varkappa_{t}^{\prime}=\phi\left(\varkappa_{t}\right)$ : $\varkappa_{t}$ is a lower bound for $k_{t}$, hence satisfies a fortiori ass. 1: $\exists \varepsilon>0: a^{t} \varkappa_{t} \underset{t \rightarrow-\infty}{\longrightarrow}$, with $a=e^{-\phi_{\infty}^{\prime}-\varepsilon}>1$. And $\phi\left(\varkappa_{0}\right)<0$.

Thus, by lemma $2, \varkappa_{t} \rightarrow \infty$, hence $\frac{\varkappa_{t}^{\prime}}{\varkappa_{t}} \rightarrow \phi_{\infty}^{\prime}$ : for $t \leq t_{0}$, $\frac{\varkappa_{t}^{\prime}}{\varkappa_{t}}<\phi_{\infty}^{\prime}+\varepsilon$, so $\varkappa_{t} \geq \varkappa_{t_{0}} e^{\left(\phi_{\infty}^{\prime}+\varepsilon\right)\left(t-t_{0}\right)}$, contradicting $a^{t} \varkappa_{t} \underset{t \rightarrow-\infty}{\varkappa_{t}} 0$.

For the second form of ass. 1, we paraphrase the end of the proof in [20, App. C, prop. 4]. $\varkappa_{t}^{\prime}=\phi\left(\varkappa_{t}\right)$ yields, with $h(x)=\int_{\bar{\kappa}}^{x} \frac{d y}{\phi(y)}$, where $\bar{\kappa}=\kappa_{P}+1, h\left(\varkappa_{t}\right)=t-a$ for some $a \in \mathbb{R}$ (since $\left.\varkappa_{t}>\kappa_{P} \forall t\right)$. By ass. $1, e^{-\phi_{\infty}^{\prime} t} \varkappa_{t} \underset{t \rightarrow-\infty}{\longrightarrow} 0$. Thus, with $x=\varkappa_{t}$ and $t_{x}=a+h(x), H_{x} \stackrel{\text { def }}{=} \ln x-\phi_{\infty}^{\prime} t_{x} \rightarrow-\infty$. But $H_{x}+a \phi_{\infty}^{\prime}-\ln \bar{\kappa}=\int_{\bar{\kappa}}^{x}\left[\frac{d z}{z}-\right.$ $\left.\phi_{\infty}^{\prime} \frac{d z}{\phi(z)}\right]=\int_{\bar{\kappa}}^{x} \frac{\phi(z)-z \phi_{\infty}^{\prime}}{z \phi(z)} d z$. Since $z \phi(z)$ is of the order $-z^{2}$ for $z \geq \bar{\kappa}$, we get that $\int_{\bar{\kappa}}^{x} \frac{\phi(z)-z \phi_{\infty}^{\prime}}{z^{2}} d z \rightarrow \infty$, hence, since the integrand is $>0$ on $\mathbb{R}_{+}, \int_{1}^{\infty} \frac{\phi(z)-z \phi_{\infty}^{\prime}}{z^{2}} d z=\infty$. Replacing now $\phi$ by $\psi$ makes a difference of $\frac{P}{z^{2}}$ in the integrand, which is integrable; then the integrand is the same with $f$ instead of $\psi$, contradicting thus the 'Strong No-Rabbit' condition in the second form of ass. 1.

This argument also proves the 'only if' part in the second item of ass. 1 .

b: $i \leq y-c+\Omega \leq f(\kappa)+\Omega^{+}$by a, so $\left\|i^{+}\right\|_{\infty, 1} \leq f(\kappa)+\left\|\Omega^{+}\right\|_{\infty, 1}$. The capital accumulation eqn. sub 2.2 .2 becomes now $e^{R t} k_{t}-e^{R t_{0}} k_{t_{0}}=\int_{t_{0}}^{t} e^{R s} i_{s} d s$, still as a Denjoy integral. But by the above the positive part of the integrand is Lebesgue integrable; so the whole integral is a Lebesgue integral: $i \in L_{1}^{\text {loc }}$. Also $k_{t}$ is locally absolutely continuous and a.e. differentiable, and is a primitive of its

\footnotetext{
${ }^{23}$ Lemma 6 in [20], plus app. C (ibidem) to deal with the weak initial condition, cannot be used here, since those proofs crucially rely on irreversibility, which does not hold in the "basic model", where current proof applies, while relying on the 1 capital good aspect.
} 
derivative $k_{t}^{\prime}$. At any point where $k$ is differentiable the left hand side in our above formula has as derivative $e^{R t}\left(R k_{t}+k_{t}^{\prime}\right)$, so this must equal a.e. the integrand in the right hand side: $i_{t}=R k_{t}+k_{t}^{\prime}$ a.e. Thus ( $k$ being a primitive of its derivative) $\int_{x-1}^{x} i_{t} d t=k_{x}-k_{x-1}-R \int_{x-1}^{x} k_{t} d t$. Hence $\int_{x-1}^{x} i_{t}^{-} d t=\int_{x-1}^{x} i_{t}^{+} d t-\int_{x-1}^{x} i_{t} d t \leq f(\kappa)+$ $\left\|\Omega^{+}\right\|_{\infty, 1}-k_{x}+k_{x-1}+R \int_{x-1}^{x} k_{t} d t, \leq f(\kappa)+\left\|\Omega^{+}\right\|_{\infty, 1}+(1+R) \kappa$ by a; thus the result.

c: $c_{t}$ is bounded by $\Omega_{t}+y_{t}+i_{t}^{-}$, hence that result.

As to $v$, since $C_{t}=N_{0} \int e^{\nu(t-s)} \bar{c}_{t-s, s} d s, L_{t}=N_{0} e^{(\gamma+\nu) t} \int \zeta_{s} e^{-\nu s} d s$, and $c_{t}=$ $C_{t} / L_{t}$, we get, with $\tilde{c}_{x, s}=e^{-\gamma(x+s)} \bar{c}_{x, s}$, that $\tilde{c}_{t} \stackrel{\text { def }}{=} \int \tilde{c}_{t-s, s} d s \leq \frac{L_{0}}{N_{0}} e^{|\nu|} c_{t}$.

By concavity of $u$, we have $\int u\left(\tilde{c}_{x, s}\right) d s \leq u\left(\int \tilde{c}_{x, s} d s\right)$ (Jensen if the latter integral is finite, trivially else), so by monotonicity of $u, u^{-1}\left(\int u\left(\tilde{c}_{x, s}\right) d s\right) \leq \int \tilde{c}_{x, s} d s$, i.e., $\left[\int \tilde{c}_{x, s}^{1-\frac{1}{\sigma}} d s\right]^{\frac{\sigma}{\sigma-1}} \leq \int \tilde{c}_{x, s} d s$. Since $v_{x} \leq e^{|\gamma|}\left[\int \tilde{c}_{x, s}^{1-\frac{1}{\sigma}} d s\right]^{\frac{\sigma}{\sigma-1}}$ we get thus $e^{-|\gamma|}$ $\int_{t-1}^{t} v_{x} d x \leq \int_{t-1}^{t} \int \tilde{c}_{x, s} d s d x \leq \int_{t-1}^{t+1} \tilde{c}_{z-s, s} d s d z=\int_{t-1}^{t+1} \tilde{c}_{z} d z \leq \frac{L_{0}}{N_{0}} e^{|\nu|} \int_{t-1}^{t+1} c_{z} d z$.

Corollary 2. In any feasible plan, $K_{t}=e^{-\delta t} \int_{-\infty}^{t} I_{s} e^{\delta s} d s$ as a Lebesgue integral.

Proof. Since $R>0$, b implies that $e^{R s} i_{s}=L_{0}^{-1} e^{\delta s} I_{s}$ is Lebesgue integrable on ]- $\left.\infty, t\right]$ $\forall t$. Use then ass. 1 to let $t_{0} \rightarrow-\infty$ in the capital accumulation eqn. sub 2.2.2.

Corollary 3. $U_{x} \leq \exp \left(\operatorname{sign}(\sigma-1)[\operatorname{sign}(1-\sigma) \beta]^{+}\right) u\left(e^{\gamma x} v_{x}\right)$ for any feasible plan. Proof. Bound $u^{-1}\left(U_{x}\right)=\left[\int e^{-\beta s} c_{x, s}^{1-\frac{1}{\sigma}} d s\right]^{\frac{\sigma}{\sigma-1}}$ in terms of $e^{\gamma x} v_{x}$.

3.2. Individual demand. Since utilities can be $\pm \infty$, marginal utility of income is not necessarily defined, so classical techniques (e.g., Lagrange theorem) do not apply, and we have to apply Lagrange-type techniques from scratch. Such utilities can be consistent with prices, income and consumption being positive and finite everywhere, as shown in lemma 4, so they have a-priori nothing pathological.

Next Fenchel-duality result is elementary; it will be used, in particular, to derive individual demand in lemma 4.

Lemma 3. (a) $\forall a>0, \forall p \in \mathbb{R}_{+}, \max _{0 \leq z \leq \infty}[a u(z)-p z]=\frac{1}{\sigma-1} a^{\sigma} p^{1-\sigma}$, where the left hand member is defined by continuity in $z$ at $\infty$.

(b) $z=\left(\frac{a}{p}\right)^{\sigma}$ is the unique maximiser.

Lemma 4. For any budget $M \in \overline{\mathbb{R}}_{+}$and price-system $p_{s}\left(s \in[0,1], p_{s} \in \mathbb{R}_{+}\right)$, let

$$
z_{s}=\frac{p_{s}}{M}, \quad \chi_{s}=e^{-\beta \sigma s} z_{s}^{1-\sigma}, \quad c_{s}^{*}=\frac{\left(e^{\beta s} z_{s}\right)^{-\sigma}}{\int_{0}^{1} \chi_{t} d t}
$$

where $\frac{0}{0}$ is defined as 0 , a negative power of 0 as $+\infty$, and $\frac{\infty}{\infty}$ is left undefined $\geq 0$.

Let also $J=\int_{0}^{1} z_{s}^{1-\sigma} d s$ and $U^{*}=\frac{\sigma}{\sigma-1}\left[\int_{0}^{1} \chi_{s} d s\right]^{\frac{1}{\sigma}}$. Then:

(a) Indirect utility (maximal - on the budget set) equals $U^{*}$ and is achieved.

(b) $U^{*} \in \mathbb{R}$ iff $J<\infty$.

(c) Demand is unique (as an equivalence class) iff either $U^{*} \in \mathbb{R}$ or $(\sigma<1$ and) $z_{s}=\infty$ a.e. Also, demand is unique $(=0)$ at all $s$ such that $z_{s}=\infty$.

(d) Whenever demand is unique, $c_{s}^{*}$ is well-defined a.e., and demand is given by the equivalence class of $c_{s}^{*}$.

Proof. Point (b) is obvious.

If $M=0$ and $p_{t}>0$ a.e., the result is obvious: $c_{t}=0$ on the budget set, so $c^{*}=0$, and if $\sigma>1$, then $U^{*}=0$, while if $\sigma<1$, then $U^{*}=-\infty$.

When $M=0, \sigma>1$, and $\lambda\left\{p_{t}=0\right\}>0$, many feasible bundles achieve $U^{*}=\infty$, so demand is not unique, hence the lemma is established in this case.

When $M=0, \sigma<1$, and $0<\lambda\left\{p_{t}=0\right\}<1$, the agent's instantaneous optimal consumption is clearly $c_{t}=\infty$ when $p_{t}=0, c_{t}=0$ otherwise; but since $\lambda\left\{p_{t}=0\right\}>0$ this gives him utility $-\infty$, so any point in his budget set is optimal, 
and the lemma is established in this case too. And if $p_{t}=0$ a.e., $c_{t}=\infty$ a.e., so $z_{t}=0$ a.e., $U^{*}=0$, and this case is covered too.

Thus the lemma is established when $M=0$. So, henceforth $M>0$.

Assume now $M<\infty$. To calculate the indirect utility, consider, after Lagrange, for $\mu>0$ the maximum of $\mathcal{L}(c) \stackrel{\text { def }}{=} \int_{0}^{1}\left[\mu e^{-\beta t} u\left(c_{t}\right)-p_{t} c_{t}\right] d t$. By lemma 3 , it equals $\frac{1}{\sigma-1} \mu^{\sigma} \int_{0}^{1} e^{-\beta \sigma t} p_{t}^{1-\sigma} d t$, and the equivalence class of $\tilde{c}_{t}=\left(\frac{\mu e^{-\beta t}}{p_{t}}\right)^{\sigma}$ is a maximiser, unique iff the maximum of $\mathcal{L}$ is finite, i.e., iff $J<\infty$ clearly.

For $J<\infty$, the budget $M_{1}=\int_{0}^{1} p_{t} \tilde{c}_{t} d t=\mu^{\sigma} \int_{0}^{1} e^{-\beta \sigma t} p_{t}^{1-\sigma} d t$ is finite.

In particular, if $0<J<\infty$, by varying $\mu$ we can obtain $M_{1}=M$ for any $0<$ $M<\infty$; so for any such $M$, and the corresponding $\mu(M)$, we obtain $\tilde{c}(\mu(M))=c^{*}$ and $U\left(c^{*}\right)=U^{*}$ as in the statement.

And $c^{*}$ is the agent's unique optimal choice given his budget $M$ : for any $c^{\prime} \neq c^{*}$ s.t. $\left\langle p, c^{\prime}\right\rangle \stackrel{\text { def }}{=} \int p_{t} c_{t}^{\prime} d t \leq M$, the integrability of $p c^{\prime}$ implies $\mu U\left(c^{\prime}\right)-\left\langle p, c^{\prime}\right\rangle=\mathcal{L}\left(c^{\prime}\right)<$ $\mathcal{L}\left(c^{*}\right)=\mu U\left(c^{*}\right)-\left\langle p, c^{*}\right\rangle=\mu U^{*}-M$, where the strict inequality is by the uniqueness property of the maximiser $\tilde{c}$. So $\left\langle p, c^{\prime}\right\rangle \leq M$ and $c^{\prime} \neq c^{*}$ implies $U\left(c^{\prime}\right)<U^{*}$.

Thus the statement is proved for $0<J<\infty$ and $M<\infty$.

When $0<M<\infty, J=0$ means, since $p_{t}<\infty$ a.e., that $\sigma<1, p_{t}=0=z_{t}$ a.e., so $c=\infty=c^{*}$, and the utility $U^{*}=0$ is attained, thus this case is settled too.

To summarize, the lemma is proved when $M<\infty$ and either $M=0$ or $J<\infty$.

If $J=\infty$ (and, recall, $0<M<\infty$ ), then, for $\sigma<1, \mathcal{L}(c)=-\infty \forall c$. So, whenever $p_{t} c_{t}$ is integrable, the indirect utility is $\int_{0}^{1} \mu e^{-\beta t} u\left(c_{t}\right) d t=-\infty$, hence all points in the budget set are utility maximisers. Thus this case is solved too.

So, in case $0<M<\infty$ it remains to prove the lemma for $J=\infty$ and $\sigma>1$, which then is assumed to hold for the next two paragraphs.

Consider the indirect utility function $V(M)$ (for fixed price system $p$ ): by homogeneity, it must be of the form $v u(M)$ for some $v \geq 0$. Assume now $v<\infty$. Then by lemma 3 for any $\mu>0, \max _{0<M<\infty}(\mu V(M)-M)=\frac{1}{\sigma-1}(\mu v)^{\sigma}$. So for any $c$ such that $p_{t} c_{t}$ is integrable we get $\mathcal{L}(c)=\int_{0}^{1}\left[\mu e^{-\beta t} u\left(c_{t}\right)-p_{t} c_{t}\right] d t \leq \frac{1}{\sigma-1}(\mu v)^{\sigma}$. As was shown above, the unique maximiser of $\mathcal{L}$ is $\tilde{c}(\mu)$. Let then $c_{t}^{N}=\min \left(\frac{N}{p_{t}}, \tilde{c}_{t}(\mu)\right)$. $p_{t} c_{t}^{N}$ being integrable, $c_{t}^{N}$ satisfies our bound above. If $p_{t}=\infty$ then $\tilde{c}_{t}(\mu)=0$ and so is $\frac{N}{p_{t}}$ for any $N$. Since then $c_{t}^{N}$ increases to $\tilde{c}_{t}(\mu)$, the corresponding integrands in $\mathcal{L}\left(c_{t}^{N}\right)$ are non-negative and increase to that for $\tilde{c}_{t}(\mu)$ : by the monotone convergence theorem, $\tilde{c}_{t}(\mu)$ still satisfies the same inequality, i.e., as seen above, $\frac{1}{\sigma-1} \mu^{\sigma} \int_{0}^{1} e^{-\beta \sigma t} p_{t}^{1-\sigma} d t \leq \frac{1}{\sigma-1}(\mu v)^{\sigma}<\infty$, contradicting $J=\infty$.

Thus $v=\infty$, i.e., $V(M)=+\infty$. We claim that therefore, $\forall M: 0<M<\infty$, there exist (many) $c$ in the budget set with $U(c)=\infty$. Indeed, note first that there exists a partition of $[0,1]$ in 2 Borel subsets of equal Lebesgue measure such that $J=$ $\infty$ on each (e.g., consider the distribution of the integrand of $J$, and on each atom use non-atomicity of Lebesgue measure). Next re-use this on one of the subsets, etc., to obtain a Borel partition into a sequence $B_{n}$ with $\lambda\left(B_{n}\right)=2^{-n}$ s.t. $J=\infty$ on each $B_{n}$. Hence for each $B_{n}$ the supremum of utility derived on that subset of time with a strictly positive finite budget should be infinite by the argument above. Choose thus for each $n$ a consumption plan $\hat{c}^{n}$ on $B_{n}$ costing $\leq 2^{-n} M$ and with $\int_{B_{n}} u\left(\hat{c}_{t}^{n}\right) d t \geq 1$ : the resulting total consumption plan $\hat{c}$ costs $\leq M$ and has infinite utility (and so does $\theta \hat{c}$ for $0<\theta<1)$ : $U^{*}=+\infty$ is attained and demand is multivalued.

Remains thus only the case $M=\infty$. Then, since $p<\infty$ a.e., for $\sigma>1, U^{*}=\infty$ and demand is multivalued. While for $\sigma<1, U^{*}=0$ and $c^{*}=\infty$.

\subsection{Optimality of production.}

Lemma 5. (a) $p_{t}^{C} \leq p_{t}^{Y} \geq p_{t}^{I}$ a.e. 
(b) Wherever the constraint that consumption cannot be transformed into output is not binding $p_{t}^{C}=p_{t}^{Y}$ a.e. Wherever the constraint that investment cannot be transformed into output is not binding, $p_{t}^{I}=p_{t}^{Y}$ a.e.

(c) Let $\mathfrak{c}(r, w)=\inf _{(K, L) \in \mathbb{R}_{+}^{2}}\{r K+w L \mid F(K, L)=1\}$ on $[0, \infty]^{2} \mathfrak{c}$ is positively homogeneous of degree one, concave and continuous on $\mathbb{R}_{+}^{2} \cdot{ }^{24}$

Assume $p_{t}^{Y}<\infty$. Then maximal profit of the production firms at time $t$ is 0 iff $w_{t}, r_{t} \geq 0$, and $\mathfrak{c}\left(r_{t}, e^{-\gamma t} w_{t}\right) \geq p_{t}^{Y} \geq 0$.

Proof. a-b: The zero profit condition in merchandising implies that $p_{t}^{I} \leq p_{t}^{Y}$ and $p_{t}^{C} \leq p_{t}^{Y}$ a.e. (b) then follows.

c: $\mathfrak{c}$ is u.s.c., and positively homogeneous of degree one and concave on $\mathbb{R}_{+}^{2}$, as an inf of continuous linear functions. Continuity on $\mathbb{R}_{+}^{2}$ is then standard.

0 profits can always be achieved with $K=L=Y=0$.

Thus, using $r, w, p$ for $r_{t}, e^{-\gamma t} w_{t}, p_{t}^{Y}$, we need that $\forall(K, L) \in \mathbb{R}_{+}^{2}, Y \leq F(K, L) \Rightarrow$ $r K+w L-p Y \geq 0$. For $(K, L, Y)=(0,0,-1)$ (resp., $(1,0,0)$ and $(0,1,0))$ this yields $p \geq 0$ (resp., $r \geq 0$ and $w \geq 0$ ), and then remains to express the condition when $Y=F(K, L)>0$ : homogeneity of $F$ of degree 1 yields then the result.

We now reformulate the condition of lemma 5.c; we will need both formulations.

Corollary 4. Let $g(k)=p f(k)-r k$. Assume the price of output, $p$, is finite. Then maximal profits of the production firm are 0 iff $p \geq 0, g_{\infty}^{\prime} \leq 0$ and $\sup _{k \geq 0} g(k) \leq w$.

Proof. $p \geq 0$ is by lemma 5.c. Next, profits are non-negative if for all feasible $(Y, K, L), p Y-w L-r K \leq 0$. Not using free-disposal, i.e., setting $Y=F(K, L)$ can only increase profits, hence it is sufficient to verify that $p F(K, L)-w L-r K \leq 0$. If $L=0$, this inequality is equivalent to $p F(K, 0)-r K \leq 0$, which holds trivially for $K=0$. For $K>0, F(K, 0)=K \lim _{k \rightarrow \infty} \frac{f(k)}{k}=K f_{\infty}^{\prime}$, so the condition becomes $p f_{\infty}^{\prime}-r \leq 0$, i.e., $g_{\infty}^{\prime} \leq 0$. Observe this implies $r \geq 0$.

If $L>0$, the condition becomes $p f(k)-r k-w \leq 0 \forall k$. For $k=0$ this implies $w \geq 0$. Since $p \in \mathbb{R}$ and $r \geq 0$, this is equivalent to $w \geq g(k) \forall k$ (treat separately the case where $r=\infty$, and similarly for $w)$, hence $w \geq \sup _{k \geq 0} g(k)$.

Lemma 6. Let $g(k)=p f(k)-r k$. Then, under the conditions of cor. 4:

(a) Any profit-maximising $L$ is 0 iff $g(k)<w \forall k$.

(b) Any profit-maximising $K$ is 0 iff $g(k)<w \forall k>0$ and $g_{\infty}^{\prime}<0$.

(c) Any profit-maximising $Y$ is 0 iff $g(k)<w \forall k>0$ and either $f_{\infty}^{\prime}=0$ or $g_{\infty}^{\prime}<0$ and either $f(0)=0$ or $g(0)<w$.

Proof. a: We need that $L>0 \Rightarrow p Y-r K-w L<0$ for any feasible $(Y, K, L)$. Since $p \geq 0$ (cor. 4 ), we can assume $Y=F(K, L)$. Then, by homogeneity, one can as well divide by $L>0$ : so $p f(k)-r k-w<0 \forall k$.

b: Similarly we need $K>0 \Rightarrow \forall L, p F(K, L)-r K-w L<0$. The case $L>0$ yields by homogeneity that $g(k)<w \forall k>0$, and $L=0$ that $K>0 \Rightarrow p F(K, 0)-$ $r K<0$, i.e., $p F(1,0)-r<0$, hence $g_{\infty}^{\prime}<0$ since $F(1,0)=f_{\infty}^{\prime}$ (continuity of $F$ ).

c: Now we need $F(K, L)>0 \Rightarrow p F(K, L)-r K-w L<0$. But $F(K, L)>0$ implies that either $K>0$ or $L>0$. Assume first $L>0$ : we get that $f(k)>0 \Rightarrow$ $g(k)<w$. Thus $g(k)<w \forall k>0$ and either $f(0)=0$ or $g(0)<w$. Dually $K>0$ yields, if also $L>0, g(k)<w \forall k>0$, and if $L=0, F(1,0)>0 \Rightarrow p F(1,0)-r<0$, i.e., either $f_{\infty}^{\prime}=0$ or $g_{\infty}^{\prime}<0$.

\footnotetext{
${ }^{24}$ In fact, the unit-cost function $\mathfrak{c}$ is continuous wherever finite. Also, its definition implies that $\int \mathfrak{c}(\tilde{r}, \tilde{w}) \leq \mathfrak{c}\left(\int \tilde{r}, \int \tilde{w}\right)$ for any $[0, \infty]$-valued measurable functions $\tilde{r}$ and $\tilde{w}$ ("strong concavity").
} 
Corollary 5. Let $D=\left\{x \mid M_{x} \stackrel{\text { def }}{=} \int_{0}^{1}\left(p_{x+s}^{C} \omega_{x, s}+w_{x+s} \zeta_{s}\right) d s\right.$ is well-defined $\} .{ }^{25}$ A.e. on $D, M_{x} \geq 0$ and $U_{x}^{*}$ of lemma 4 is well-defined and is the equilibrium utility.

Proof. $D$ is Lebesgue measurable by the joint measurability of $\omega$, and the same implies $M_{x}$ is Lebesgue measurable there. $M_{x} \geq 0$ a.e. there is an obvious equilibrium condition. That $U_{x}^{*}$ is well-defined on $D$ and is the equilibrium utility follows then from lemma 4.a because $p_{t}^{C}<\infty$ by lemma 5 .a and def. 1 .

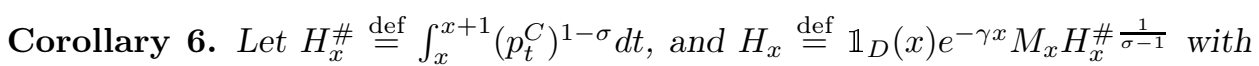
$0 \cdot \infty=0$. Then:

(a) $\|H\|_{\infty, 1} \leq K+L\|\Omega\|_{\infty, 1}$ for some $K, L \in \mathbb{R}$.

(b) $M_{x}<\infty$ a.e. on $D$.

(c) $D_{1} \stackrel{\text { def }}{=}\left\{x \in \mathbb{R} \mid p_{t}^{C}=0\right.$ a.e. on $\left.[x, x+1]\right\}$ is discrete and closed in $\mathbb{R} ; D_{1} \subseteq D$.

(d) $H_{x}^{\#}>0$ outside $D_{1}$, and is l.s.c. It is $>0$ everywhere if $\sigma>1$.

(e) If $\sigma>1, \exists D_{0} \subseteq D$ s.t. $D \backslash D_{0}$ is negligible and $p_{t}^{C}>0$ a.e. on $D_{0}+[0,1]$.

Proof. By cor. 3 and $5, U_{x}^{*} \leq K u\left(e^{\gamma x} v_{x}\right)$ with $K=\exp \left(\operatorname{sign}(\sigma-1)[\operatorname{sign}(1-\sigma) \beta]^{+}\right)$, and by lemma $4, U_{x}^{*}=\frac{\sigma}{\sigma-1}\left[\int e^{-\beta \sigma s} z_{x, s}^{1-\sigma} d s\right]^{\frac{1}{\sigma}}$. Applying $u^{-1}$ on both sides of the resulting inequality we get $\left[\int e^{-\beta \sigma s} z_{x, s}^{1-\sigma} d s\right]^{\frac{1}{\sigma-1}} \leq K^{\prime} e^{\gamma x} v_{x}$ with $K^{\prime}=\exp \left(\sigma\left(\frac{\beta}{1-\sigma}\right)^{+}\right)$. Now, $\left[\int z_{x, s}^{1-\sigma} d s\right]^{\frac{1}{\sigma-1}} \leq L\left[\int e^{-\beta \sigma s} z_{x, s}^{1-\sigma} d s\right]^{\frac{1}{\sigma-1}}$ with $L=\exp \left(\frac{\sigma}{\sigma-1}(\beta \operatorname{sign}(\sigma-1))^{+}\right)$; and thus $\left[\int z_{x, s}^{1-\sigma} d s\right]^{\frac{1}{\sigma-1}} \leq L^{\prime} e^{\gamma x} v_{x}$ with $L^{\prime}=\exp \left[\sigma\left(\frac{\beta^{-}}{\sigma-1}+\frac{\beta^{+}}{\sigma-1 \mid}\right)\right]$. Denote the first integral by $J_{x}$ : in particular $H_{x}^{*} \stackrel{\text { def }}{=} e^{-\gamma x} J_{x}^{\frac{1}{\sigma-1}}$ is well-defined and locally integrable wherever $M_{x}$ is well-defined, where by lemma $4, z_{x, s}=\frac{p_{x+s}^{C}}{M_{x}}$ with $\frac{0}{0}=0$.

If $M_{x}=\infty$, then $z_{x, s}=0$ a.e., so $H_{x}^{*}=\infty$ : this can only happen on a negligible set, since $H_{x}^{*} \leq L^{\prime} v_{x}$ and $v$ is locally integrable by prop. 1.c. Hence b. Then clearly $H_{x}^{*}=H_{x}$ when $M_{x}>0$. If $M_{x}=0$, then, for $\sigma<1, H_{x}^{*}<\infty$ implies $p_{t}^{C}$ is not a.e. null on $[x, x+1]$, and thus $H_{x}^{*}=0,=H_{x}$. And for $\sigma>1, H_{x}^{*}<\infty$ implies $p_{t}^{C}>0$ a.e. on $[x, x+1]$ (implying thus e), and then $H_{x}^{*}=0,=H_{x}$ again.

Thus $H_{x} \leq L^{\prime} v_{x}$, and since prop. 1 implies $\|v\|_{\infty, 1} \leq K+L\|\Omega\|_{\infty, 1}$ for some $K, L \in \mathbb{R}$, a follows.

As to d, lower semi-continuity of $H^{\#}$ is clear, since it is the limit of the increasing sequence of continuous functions $\int_{x}^{x+1} \min \left\{n,\left(p_{t}^{C}\right)^{1-\sigma}\right\} d t$. If $\sigma>1, H^{\#}>0$ follows from $p_{t}^{C}<\infty$ a.e. And else, we just saw that a.e. on $D, p_{t}^{C}>0$ on a non-negligible subset of $[x, x+1]$. Clearly $D_{1} \subseteq D$, so the above implies $D_{1}$ is negligible if $\sigma<1$, and e implies the same for $\sigma>1$. Thus $x \neq y \in D_{1}$ implies $|x-y|>1: D_{1}$ is closed and discrete. Thus also c.

\subsection{Optimal investment.}

Lemma 7. Investment firms maximise profits at a feasible point iff $p_{t}^{I}=p_{t}<\infty$ a.e., and $\varsigma(t) \stackrel{\text { def }}{=} e^{-\delta t} p_{t}-\int_{t}^{\infty} r_{s} e^{-\delta s} d s \geq 0$ is decreasing, and constant where $K_{t}>0$.

Comment 10. Observe the condition depends only on aggregates. Indeed, prop. 2 and 3 in app. B.3 imply that there always exists a disaggregation, satisfying the strictest conditions, and for which the two notions of profits are equivalent (lemma 23): the one of app B.4 used for necessity, requiring bounded variation of the firm's capital and the more general one (in app. B.6), used for sufficiency.

Proof. We first prove the conditions are necessary. To show $p_{t}<\infty \forall t$, assume else $p_{t_{0}}=\infty$. But then investment firms alive just before $t_{0}$ can make infinite profits. Indeed, consider (ass. 2.c) $\varepsilon>0$ s.t. $\mu\left\{f \mid t_{0}^{f} \leq t_{0}-\varepsilon, t_{1}^{f} \geq t_{0}\right\}>0$; since $p_{t}^{I} \leq p_{t}^{Y}<\infty$ a.e., $\exists M<\infty: \lambda\left\{t \in\left[t_{0}-\varepsilon, t_{0}\right] \mid p_{t}^{I} \leq M\right\}>0$. So if those firms

\footnotetext{
${ }^{25}$ Since $p^{C}$ (by lemma 5.a and def. 1) and $\omega$ are a.e. finite, and $\zeta \geq 0, w \geq 0$ (lemma 5.c), the integrand is a.e. well-defined, so this means either its positive or negative part is integrable.
} 
invest at unit rate during this set they get a positive amount of capital at finite cost, that can be re-sold for $\infty$ at $t_{0}$; contradiction.

Next, $r_{t}$ is locally integrable: if it was not integrable on $\left[t_{0}-\varepsilon, t_{0}\right]$, choose $0<\delta<\varepsilon$ s.t. $\mu\left\{f \mid t_{0}^{f} \leq t_{0}-\varepsilon, t_{1}^{f} \geq t_{0}\right\}>0$ (ass. 2.c), and let those firms buy some capital at $t_{0}-\varepsilon$, cash its returns until $t_{0}$, and sell it then, yielding infinite profit, since $p_{t}<\infty$. Similarly with $G_{t_{0+}}$ if $r_{t}$ is not integrable on $\left[t_{0}, t_{0}+\varepsilon\right]$.

Consider a deviation (as in lemma 22 of app. B.2) where firms $f$ s.t. $K_{t}^{f} \geq \varepsilon$ for $a<t<b$ buy, with $\delta K_{t}^{f}=\xi e^{-\delta t} \mathbb{1}_{] a, b[}, \delta K_{a_{+}}^{f}$ additional capital at time $a$, and sell $\delta K_{b_{-}}^{f}$ at time $b$, cashing the returns in between. Then $\delta \pi^{f}=\xi(g(b)-g(a))$, with $g(t)=e^{-\delta t} p_{t}+\int_{0}^{t} r_{s} e^{-\delta s} d s,<\infty$ since $r_{s}$ is locally integrable and $p_{t}<\infty$.

Fix now $t$, and assume either $K_{t}>0$ or $\xi>0$. If $\xi \geq 0$, the above deviation is always feasible. Else, by assumption 3.d, $\exists \varepsilon>0: K_{x}^{f}>\varepsilon$ on $[t, t+\varepsilon] \times G$, with $\mu(G)>0$; and then the deviation is feasible $\forall f \in G, \forall \xi: \xi \geq-\varepsilon e^{\delta t} e^{\varepsilon \delta^{-}}$, $\forall a, b: t \leq a<b \leq t+\varepsilon$. So, since $\mu(G)>0$, absence of profitable deviations implies $g$ is decreasing on $[t, t+\varepsilon]$ and is constant there if $K_{t}>0$. Similarly on $[t-\varepsilon, t]$, thus, $t$ being arbitrary, $g$ is decreasing, and is constant wherever $K_{t}>0$.

So $\forall t \geq 0 g(0) \geq \int_{0}^{t} r_{s} e^{-\delta s} d s$, and $g(0)<\infty$, hence $\int_{0}^{\infty} r_{s} e^{-\delta s} d s<\infty$; subtracting this quantity from $g(t)$ we get that $\varsigma(t)=e^{-\delta t} p_{t}-\int_{t}^{\infty} r_{s} e^{-\delta s} d s$ is decreasing and (letting $t \rightarrow \infty) \geq 0$, and is constant wherever $K_{t}>0$.

Next we show that $p_{t}^{I}=p_{t}$ a.e. Else, $p$ being Borel by the previous conclusion, there exists, by Lusin's theorem, a compact set $K$ with $\lambda(K)>0$ to which $p_{t}^{I}$ and $p_{t}$ have a continuous restriction, with either (1) $p_{t}>p_{t}^{I} \forall t \in K$ or (2) $p_{t}<p_{t}^{I} \forall t \in K$.

Let $K_{n}=K \cap L_{n}$ with $L_{n}=\left[T-n^{-1}, T\right]$ in case 1 and $\left[T, T+n^{-1}\right]$ in case 2 , for some $T \in K$ s.t. $\forall n, \lambda\left(K_{n}\right)>0$. $G_{n}$ is the set of firms alive on $L_{n} ; \mu\left(G_{n}\right)>0$ for $n \geq n_{0}$ by ass. 2.c. Let firms buy/sell additional investment $\delta I_{t}^{n}=\xi \mathbb{1}_{G_{n}}(f) \mathbb{1}_{K_{n}}(t)$, where $\xi \stackrel{\text { def }}{=} \operatorname{sign}\left(p_{T}-p_{T}^{I}\right)$, and, at time $T$, sell the additional accumulated capital, resp., buy additional capital such that it will be exactly offset by $\delta I^{n}$.

So $\delta K_{t}^{n}=e^{-\delta t} \mathbb{1}_{L_{n}}(t) \int_{T \pm n^{-1}}^{t} e^{\delta s} \delta I_{s}^{n} d s$. Observe that $\delta K_{t}^{n}$ is of bounded variation and $\geq 0$, and jointly measurable (in $(f, t)$ ) by the same property of $\delta I_{t}^{n}$.

By the formula sub claim 10 in app. B.2, the induced variation in profit is:

$$
\delta \pi^{n}=\xi p_{T} \delta K_{T}^{n}+\int_{L_{n}}\left(r_{t} \delta K_{t}^{n}-p_{t}^{I} \delta I_{t}^{n}\right) d t
$$

The last term in the integrand is jointly integrable in $(t, f)$, by the same property of $\delta I_{t}^{n}$ and the continuity of $p^{I}$ on the compact set $K, \delta I_{t}^{n}$ being 0 outside of $K$.

And the first term, $r_{t} \delta K_{t}^{n}$, is the integral over $s$ of $\xi r_{t} e^{-\delta t} J(s, t) e^{\delta s} \delta I_{s}^{n}$, with $J(s, t)=\mathbb{1}_{T-n^{-1} \leq s \leq t \leq T}$ in case 1 and $\mathbb{1}_{T \leq t \leq s \leq T+n^{-1}}$ in case 2 , and where all terms are clearly jointly measurable and have a constant sign. Linearity of the integral allows then to integrate both terms separately (the second being integrable); Fubini's theorem allows then, for the first term, to integrate first over $t$, yielding for this term $\int_{L_{n}} e^{\delta s} \delta I_{s}^{n}\left[\int_{s}^{T} e^{-\delta t} r_{t} d t\right] d s$. Replacing also $\delta K_{T}^{n}$ by its value, we get thus, re-using linearity of the integral, $\delta \pi^{n}=\int_{L_{n}}\left[p_{T} e^{\delta(t-T)}+e^{\delta t} \int_{t}^{T} e^{-\delta s} r_{s} d s-p_{t}^{I}\right] \delta I_{t}^{n} d t=$ $\xi \mathbb{1}_{G_{n}}(f) \int_{K_{n}}\left[p_{T} e^{\delta(t-T)}+e^{\delta t} \int_{t}^{T} e^{-\delta s} r_{s} d s-p_{t}^{I}\right] d t$.

Since $\mu\left(G_{n}\right)>0$, the coefficient of $\mathbb{1}_{G_{n}}$ must be $\leq 0$. Since $\lambda\left(K_{n}\right)>0, \exists t_{n} \in K_{n}$ s.t. $\xi\left[p_{T} e^{\delta\left(t_{n}-T\right)}+e^{\delta t_{n}} \int_{t_{n}}^{T} e^{-\delta s} r_{s} d s-p_{t_{n}}^{I}\right] \leq 0$. Since $K_{n}$ shrinks to $\{T\}, t_{n} \rightarrow T$, so, by continuity of $p^{I}$ and $p$ on $K$, we get in the limit $\xi\left(p_{T}-p_{T}^{I}\right) \leq 0$, contradiction.

Remains to show that with such prices, investment firms make zero profits, and can't do better. We allow here for any production plans satisfying def. 11 in app. B.6. Accordingly the corresponding profits are $\pi=\int\left(r_{t}-p_{t} \delta\right) K_{t} d t+f K_{t} d p_{t}+\int\left(p_{t}-\right.$ $\left.p_{t}^{I}\right) I_{t} d t$. Since $p^{I}=p$ a.e., the last term is zero. And the formula for $p_{t}$ implies $d p_{t}=\left(p_{t} \delta-r_{t}\right) d t+e^{\delta t} d \varsigma_{t}$, so $\pi=f e^{\delta t} K_{t} d \varsigma_{t}$. Since $\varsigma$ is decreasing and $K_{t} \geq 0$, indeed $\pi \leq 0$ for any plan in the production set. 
If however $K_{t}^{f}$ is feasible, as an aggregate production plan, so $K_{t}=\int K_{t}^{f} \mu(d f)$ is continuous (cor.2). Further by ass. 3.a $K_{t}^{f}$ is $\mu \otimes\left(\left|d p_{t}\right|+\lambda(d t)\right)$-measurable and by ass. 3.d $p_{t} \neq p_{t_{+}} \Rightarrow \lim _{\varepsilon \searrow 0} K_{t+\varepsilon}^{f}=K_{t_{+}}^{f}$ a.e. and $p_{t_{-}} \neq p_{t} \Rightarrow \lim _{\varepsilon \searrow 0} K_{t-\varepsilon}^{f}=K_{t_{-}}^{f}$ a.e. Let then $Z=\left\{t \mid K_{t}=0\right\}: \forall t \in Z, K_{t}^{f}=0$ a.e., and, by continuity of $K_{t}$ and Fatou's lemma, also, at discontinuities of $p_{t}, K_{t_{-}}^{f}=K_{t_{+}}^{f}=0$ a.e. and the convergence is in $L_{1}$, so all assumptions of lemma 25 are satisfied $-p_{t}^{I} I_{t}^{f}$ is integrable since $p_{t}^{I}=p_{t}$ is locally bounded, and for $r_{t} K_{t}^{f}$ : since the integrand is $\geq 0$, first integrate over $f$ (Fubini), then use that $K_{t}$ is locally bounded and $r_{t}$ locally integrable. Since $d \varsigma_{t}$ is carried by $Z$, clearly the aggregate $\pi=0$, so by lemma $25 \pi^{f}=0 \mu$-a.e.

Comment 11. As the "hot potato" example (app. B.1) shows, ass. 3.d is clearly needed to derive the lemma. Without it, one cannot deduce the constancy of $\varsigma_{t}$, even where $K_{t}>0$ (though one can obtain that there $\varsigma(t)$ is the sum of countably many jumps, i.e., its continuous part is 0 , getting then similarly in thm. 1 the analog of thm. 1(3) for such $\varsigma_{t}$ ). So the example presents really the pure form of the difficulty.

Corollary 7. $p^{Y}=p$ implies $p$ is locally bounded away from zero.

Proof. If, on the contrary, $p$ is not locally bounded away from zero, then, by lemma 7 , there exists a finite $t_{0}$ such that $\lim _{t \searrow_{0}} p_{t}=0$ and $p_{t}=0$ for $t>t_{0}$. But this is impossible since $p^{Y} \geq p^{C}$ a.e. by lemma 5.a and $p^{C}$ is distinct from zero on a non-negligible set, by lemma 6.c.

\subsection{First conclusions about equilibria.}

Lemma 8. Assume $\complement D$ is negligible and either $\sigma>1$ or $\left(p^{C}\right)^{1-\sigma}$ is locally integrable. Then, for a.e. agent, any addition to income increases his life-time utility.

Proof. Let $V_{x}(M) \stackrel{\text { def }}{=} u(M)\left(\int_{0}^{1} e^{-\beta \sigma s}\left(p_{x+s}^{C}\right)^{1-\sigma} d s\right)^{\frac{1}{\sigma}}, 0 \leq M<\infty . V_{x}(M)$ is the indirect utility of consumer born at $x$ with income $M$ by lemma 4.a. The integral is $>0$ a.e. (cor. 6.d), and finite for $\sigma<1,\left(p^{C}\right)^{1-\sigma}$ being locally integrable, so $V_{x}$ is well-defined even on $[0, \infty]$ and is strictly increasing in $M$. For $\sigma>1$, assume first it is finite. Then $V_{x}$ is strictly increasing in $M$ as above. While if the integral is infinite, cor. 6.a implies that $H<\infty$ a.e., so $M_{x}=0$. Since $p_{t}^{C}>0$ a.e. (cor. 6.e), this implies in lemma 4 that $z_{t}=\infty$ a.e., so $J_{x}=0$ and hence the equilibrium utility $U_{x}^{*}$ (cor. 5$)$ is well-defined and $=0$. On the other hand for $M>0$, the integral being infinite implies that $V_{x}(M)$ is so too. Thus in this case too any increase in budget increases utility - from 0 to $+\infty$. Finally, since $M_{x}<\infty$ a.e. (cor.6.b), any addition to $M_{x}$ increases the budget, and hence the utility.

Corollary 8. Assume $\complement D$ is negligible and either $\sigma>1$ or $\left(p^{C}\right)^{1-\sigma}$ is locally integrable. Then a.e. $w_{t}>0$ implies full employment (labour contracted $=L_{t}$ ).

Proof. Assume contrary to the statement that the set $T=\left\{t \in \mathbb{R} \mid L_{t}=0, w_{t}>0\right\}$ is of positive measure. Let $P=\left\{s \mid \zeta_{s}>0\right\}$ and $X=\left\{x \mid h_{x} \stackrel{\text { def }}{=} \lambda(P \cap(T-x))>\right.$ $0\}$. $X$ is a set of consumers who can increase their income by supplying labour and hence by lemma 8 can improve their utility. Since $\int h_{x} d x=\lambda(P) \lambda(T)>0, X$ is non-negligible, contradiction.

Lemma 9. (a) $C_{t}$ is locally integrable, and $p_{t}$ is locally bounded.

(b) $\complement D$ is negligible if either $p^{C}$ is locally bounded or $\omega \geq 0$.

(c) Assume $\complement D$ negligible. Then $p_{t}$ is locally bounded away from 0 unless $f$ is bounded, $\sigma<1$, and either $\left(p^{C}\right)^{1-\sigma} \notin L_{1}^{\text {loc }}$ or $\nexists t_{0}: f^{\prime}\left(k_{t}\right)>0$ a.e. on $\left[t_{0}, \infty[\right.$.

Remark 12. When it is known that $p^{Y}=p^{I}$, cor. 7 is easier to use to establish that $p_{t}$ is locally bounded away from 0 , but point (c) will be used in lemma 12 . 
Proof. b is clear. For a: $C_{t}$ is locally integrable by prop. 1.c. That $p_{t}$ is locally bounded follows from lemma 7 .

For c: Assume that $p_{t}$ is not locally bounded away from zero, then by lemma 7 , since $\varsigma$ is decreasing, there is $t_{0}<\infty$ such that $\lim _{t \searrow t_{0}} p_{t}=0$. The same equation in lemma 7 implies then $\varsigma_{s}=r_{s}=0$ a.e. on $] t_{0}, \infty[$.

By cor.6.c, $p_{t}^{C}$ cannot be 0 a.e. on an interval of length $>1$. So (lemma 5.a) $T \stackrel{\text { def }}{=}\left\{t>t_{0} \mid p_{t}^{Y}>0\right\}$ is non-null. By cor. $4, r_{t}=0$ implies there $\sup _{k>0} f_{k} \leq$ $e^{-\gamma t} w_{t} / p_{t}^{Y}$. And $w_{t}<\infty$ a.e.: else, since $M_{x}$ is well-defined a.e., one would have $M_{x}=\infty$ a.e. on the non-empty open set $\left\{x \mid \lambda\left\{s \mid w_{x+s}=\infty, \zeta_{s}>0\right\}>0\right\}$, contradicting cor.6.b. So $f$ is bounded, and we can henceforth assume that either $\sigma>1$ or $\left(p^{C}\right)^{1-\sigma} \in L_{1}^{\text {loc }}$.

Then, for a.e. $t \in T, \exists k: f_{k} \geq e^{-\gamma t} w_{t} / p_{t}^{Y}$. Indeed, if $e^{-\gamma t} w_{t} / p_{t}^{Y}>f_{k} \forall k$, labour contracted would be 0 by lemma 6 .a, contradicting cor. 8 since $w_{t}>0$.

Thus $\exists \bar{k}, 0<\bar{k}<\infty: f_{k}=f_{\bar{k}}$ iff $k \geq \bar{k}$ and, a.e. on $T, e^{-\gamma t} w_{t} / p_{t}^{Y}=f_{\bar{k}}$. So $w_{t}>0$ a.e. on $T$. Cor. 8 implies contracted labour $=L_{t}$ where $w_{t}>0$. Thus, a.e. on $T, k_{t}<\bar{k}$ would yield negative profits in production, so a.e. on $T, k_{t} \geq \bar{k}$, i.e., $f^{\prime}\left(k_{t}\right)=0$.

We claim finally that $\sigma<1$. Indeed, else, by cor. 6.e $p_{t}^{C}>0$ a.e., so $T=\left[t_{0}, \infty[\right.$. Since $p_{t}^{Y} \geq p_{t}^{C}>0=p_{t}^{I}$, no output nor consumption good can be transformed into investment there: $I_{t}=0$ there, hence $k_{t}=k_{t_{0}} e^{-R\left(t-t_{0}\right)}$ will at some time get below $\bar{k}$, contradiction.

Notation 3.2. For $h: \mathbb{R} \rightarrow \mathbb{R}, \underline{D}_{x} h$ denotes the lower derivative of $h$ at $x$, i.e., liminf $y \neq x, y \rightarrow x \frac{h(y)-h(x)}{y-x}$, and similarly for the upper derivative $\bar{D}$.

Remark 13. In the following, we also select w.l.o.g. canonical representatives within equivalence classes, so as to make maximisation hold everywhere instead of just a.e.

Lemma 10. Assume $p^{Y}=p^{I}$, and let $g_{t}=f^{\prime}\left(k_{t}\right)$. Then, $\forall t$ :

(a) (1) $0 \leq p^{C} \leq p, p$ is locally bounded and locally bounded away from 0 .

(2) $y_{t}=f\left(k_{t}\right)$

(3) $w_{t}=\left(f\left(k_{t}\right)-k_{t} f^{\prime}\left(k_{t}\right)\right) e^{\gamma t} p_{t}$, with $k f_{k}^{\prime}$ defined as 0 at 0 , by continuity.

(4) $p_{t} g_{t} \leq r_{t} \leq \delta p_{t}-\bar{D}_{t} p$, and $k_{t}>0 \Rightarrow p_{t} g_{t}=\delta p_{t}-\underline{D}_{t} p$.

(5) $p_{t}=e^{-\pi_{t}+\int_{0}^{t}\left(\delta-g_{s}\right) d s}$, with $\pi_{t}$ locally constant on $k_{t}>0$ and monotone.

(6) $M_{x} \stackrel{\text { def }}{=} \int_{0}^{1}\left[p_{x+s}^{C} \omega_{x, s}+w_{x+s} \zeta_{s}\right] d s \geq 0$ a.e., and is locally integrable.

(b) a2-a4, with $0<p_{t}<\infty$ (from a1), imply that all firms maximise profits.

(c) Given $0<p_{t}<\infty$, a5 is equivalent to the existence of $r_{t}$ s.t. a4 holds.

(d) Further, if $f^{\prime}(0)=\infty$ and $f(0)=0$, and if either $\Omega_{t} \leq 0 \forall t$ (e.g., pure transfers), or if consumption cannot be reverted to output (i.e., $(C, I, Y, L, K)$ feasible implies $\left(C^{+}, I, Y, L, K\right)$ feasible), then $K_{t}>0 \forall t$.

Remark 14. Note that, since $g \geq 0$, a5 implies that both $\pi_{t}$ and $\int_{0}^{t} g_{s} d s$ are everywhere real-valued. Indeed, both are increasing, and whenever one is finite the other must be too, since $p_{t} \in \mathbb{R}_{++}$; thus, since the integral is finite at $0, \pi_{0}$ is also finite, hence for $t>0$ they must be both finite (since else both $+\infty$ contradicts $p_{t} \in \mathbb{R}_{++}$), and similarly if $t<0$. Thus $\pi_{t}$ is $\mathbb{R}$-valued and $g$ in $L_{1}^{\text {loc }}$.

Remark 15. Also, a2 implies full output utilisation, and, iff either $k_{t}>0, k_{t} \geq$ $\max \{k \mid f$ is linear on $[0, k]\}$ or $f(0)>0$, full employment at time $t$.

Proof. a1: $p_{t}$ is locally bounded away from zero by cor. 7 , and locally bounded by lemma 7. By lemma 5.a, $0 \leq p_{t}^{C} \leq p_{t} \forall t$. Hence $p^{C}$ is locally bounded and by lemma 9.b $M_{x}$ is well-defined a.e. Thus cor. 8 is applicable.

Let $F_{L}(K, L) \stackrel{\text { def }}{=} \lim _{\varepsilon \searrow 0} \frac{F(K, L+\varepsilon)-F(K, L)}{\varepsilon}, F_{K}(K, L) \stackrel{\text { def }}{=} \lim _{\varepsilon \searrow 0} \frac{F(K+\varepsilon, L)-F(K, L)}{\varepsilon}$.

a2: $p_{t}>0$ implies $p_{t}^{Y}>0$, hence no output is disposed off. Thus if there is fullemployment, the equation holds. Else, $w_{t}=0$ by cor. 8 , and $F_{L}\left(K_{t}, L\right)=0$ at the 
contracted labour $L<L_{t}$, so $F\left(K_{t}, L\right)=F\left(K_{t}, L_{t}\right)$ and equation a2 again holds. Further, increasing $L$ to $L_{t}$ preserves the equilibrium: since marginal product of labour $\left(F_{L}\left(K_{t}, L_{t}\right)\right)$ is still zero, wages can be kept at 0 , utility of agents and profits of firms are not affected, nor is output, and still no positive profits are feasible: we can assume full employment. Hence the use of $f$ and $k$ becomes meaningful.

Claim 1. A.e., $p_{t} g_{t} \leq r_{t}$ with equality if $k_{t}>0$.

Proof of the claim. Optimal use of capital by production firms implies either $p_{t}^{Y} F_{K}\left(K_{t}, L_{t}\right)=$ $r_{t}$ or $p_{t}^{Y} F_{K}\left(0, L_{t}\right)<r_{t}$ and $K_{t}=0$. Since $L_{t}>0$ the two conditions are equivalent to $r_{t}=p_{t}^{Y} f^{\prime}\left(k_{t}\right)$ or $r_{t}>p_{t}^{Y} f^{\prime}(0)$ and $k_{t}=0$.

a3: Similarly for labour, $p_{t}^{Y} F_{L}\left(K_{t}, L_{t}\right)=e^{-\gamma t} w_{t}$ or $p_{t}^{Y} F_{L}\left(K_{t}, 0\right)<e^{-\gamma t} w_{t}$ and no labour is hired. But the latter condition implies $w_{t}>0$, in which case there is full-employment (cor. 8): contradiction. So $p_{t}\left(f\left(k_{t}\right)-k_{t} f^{\prime}\left(k_{t}\right)\right)=e^{-\gamma t} w_{t}$.

Since the equations a2-a3 hold a.e., we impose them now to hold everywhere (thereby choosing the canonical representatives, see remark 13).

This implies the following facts, used in rest of the proof. $p_{t}$ is continuous where $K_{t}>0$ by lemma 7 , so $w_{t}$ is locally bounded (since $f$ is so), and $g_{t}$ is continuous to $\overline{\mathbb{R}}_{+}$. By lemmas 7 and 5.c, $r_{t} \in L_{1+}^{\text {loc }}$.

a5: In the view of the above properties of $p_{t}$ and $r_{t}$, the equation in lemma 7 can be differentiated term by term wherever $K_{t}>0$, since there $r_{t}=p_{t} g_{t}$ is real-valued and continuous. Doing this with $z_{t}=e^{-\delta t} p_{t}$ we get $z_{t}^{\prime}=-g_{t} z_{t}$, where $z_{t}>0$ since $p_{t}>0 . g \in L_{1}^{\text {loc }}$, since $p_{t} g_{t} \leq r_{t}$ a.e., $r \in L_{1}^{\text {loc }}$ and $p$ is locally bounded away from 0 . Thus integrating from zero, with possibly a different constant $z_{0}$ in each interval where $K_{t}>0, z_{t}=z_{0} e^{-\int_{0}^{t} g_{s} d s}$. a5 always holds for an appropriate choice of $\pi_{t}$, since $p_{t}>0$ and $g$ is locally integrable; the above argument shows $\pi_{t}$ $\left(=-\ln z_{0}\right)$ is constant on each interval where $k_{t}>0$. Let $H_{t} \stackrel{\text { def }}{=} \pi_{t}+\int_{0}^{t} g_{s} d s$ : since $z_{t}=e^{-H_{t}}$ is decreasing by lemma $7, H_{t}$ is increasing, so $\pi_{t}=H_{t}-\int_{0}^{t} g_{s} d s$ has locally bounded variation; let $\pi_{t}^{a}$ and $\pi_{t}^{s}$ denote its absolutely continuous and singular parts. By lemma $7, \varsigma_{t} \stackrel{\text { def }}{=} e^{-H_{t}}-\int_{t}^{\infty} e^{-\delta s} r_{s} d s$ is decreasing; we first express that its absolutely continuous part is so, by expressing that $\varsigma^{\prime}$ exist a.e. and is a.e. $\leq 0$. Since $r_{s} \in L_{1}^{\text {loc }}$, the integral term in the right hand side is a.e. differentiable with finite derivative $e^{-\delta t} r_{t}$; similarly, since $H$ is increasing, $e^{-H_{t}}$ is a.e. differentiable with finite derivative $-H_{t}^{\prime} e^{-H_{t}}$; thus $r_{t} \leq e^{\delta t} H_{t}^{\prime} e^{-H_{t}}$ a.e., $=p_{t} H_{t}^{\prime}$ since $e^{\delta t-H_{t}}=p_{t}$, i.e., $r_{t} \leq \delta p_{t}-p_{t}^{\prime}$ a.e. Since $H_{t}^{\prime}=\pi_{t}^{\prime}+g_{t}$, we also get equivalently $r_{t} \leq p_{t} g_{t}+p_{t} \pi_{t}^{\prime}$ a.e., hence, since $0<p_{t}<\infty$, by $p_{t} g_{t} \leq r_{t}$ a.e. (by claim 1 ), that $\pi_{t}^{\prime} \geq 0$ a.e. on $g<\infty$, hence, by $g \in L_{1}^{\text {loc }}, \pi_{t}^{\prime} \geq 0$ a.e.: $\pi^{a}$ is increasing. And since $\int_{0}^{t} g_{s} d s$ is absolutely continuous, $\pi^{s}$ is the singular part of $H_{t}$, which is increasing, thus so is $\pi^{s}$.

Thus a5 is proved, and $p_{t} g_{t} \leq r_{t} \leq \delta p_{t}-p_{t}^{\prime}$ a.e. By claim 1 , replace $r_{t}$ by $p_{t} g_{t}$ when $k_{t}>0$ and by $\max \left\{r_{t}, p_{t} g_{t}\right\}$ else: this changes it only on a null set, preserving equilibrium.

b: We now prove that these inequalities, together with a2,a3 and $0<p_{t}<\infty$, imply firms maximise profits: this along with point $\mathrm{c}$ will then prove point $\mathrm{b}$. Clearly, a2 and a3, together with our conditions on $r_{t}$, express the optimality of instantaneous production. Remains to show that a5 implies the formula of lemma 7 , reversing the above argument. First, a5 implies that $g_{t} \in L_{1}^{\text {loc }}$ (cf. rem. 14). Then a5, with $z_{t}=e^{-\delta t} p_{t}=e^{-\pi_{t}-\int_{0}^{t} g_{s} d s}$, which is decreasing, yields $\varsigma_{t}=z_{t}-\int_{t}^{\infty} e^{-\delta s} r_{s} d s$, so $\varsigma_{t}$ has locally bounded variation. Since $r_{t} \leq \delta p_{t}-p_{t}^{\prime}$ a.e., $\varsigma_{t}^{\prime} \leq 0$ a.e., so the absolutely continuous part of $\varsigma$ is decreasing. And the singular part must be that of $z_{t}$, hence decreasing too. Thus $\varsigma$ is decreasing. Its equation above can be differentiated on intervals where $k_{t}>0$, because $z_{t}$ is differentiable there, $\pi_{t}$ being constant, and $r_{t}$ locally equals $p_{t} g_{t}$ a.e., which is locally bounded and continuous. Hence there we 
get $\varsigma_{t}^{\prime}=0$ identically (since $p_{t}^{\prime}=p_{t}\left(\delta-g_{t}\right)$ ), and thus $\varsigma_{t}$ is indeed constant on each interval where $K_{t}>0$. So, by lemma 7 , the investment sector maximises profits too.

We will use below the following 2 easy statements:

Claim 2. $h: \mathbb{R} \rightarrow \mathbb{R}$ is weakly increasing iff $\underline{D} h \geq 0$.

Claim 3. For $f, g: \mathbb{R} \rightarrow \mathbb{R}$, if $g\left(x_{0}\right)=y_{0}$ and $f$ is differentiable at $y_{0}$ with $f^{\prime}\left(y_{0}\right)>0$ and is weakly increasing, then $\bar{D}_{x_{0}}(f \circ g)=f^{\prime}\left(y_{0}\right) \bar{D}_{x_{0}} g$.

a4: We show that a5, with $0<p_{t}<\infty$, implies that $p_{t} g_{t} \leq \delta p_{t}-\bar{D}_{t} p$, and $k_{t}>0 \Rightarrow p_{t} g_{t}=\delta p_{t}-\underline{D}_{t} p$; it is then indeed sufficient to replace $r_{t}$ by its min with $\delta p_{t}-\bar{D}_{t} p$ to satisfy a4, since $p_{t}^{\prime}=\bar{D}_{t} p$ a.e., $p$ having locally bounded variation by a5.

By a5, $\pi_{t}=\int_{0}^{t}\left(\delta-g_{s}\right) d s-\ln p_{t}$ is increasing, hence a fortiori $\int_{0}^{t}\left(\delta-n \wedge g_{s}\right) d s-\ln p_{t}$ is so, $\forall n$. Thus by claim 2 , since the integral term is differentiable, $\delta-n \wedge g_{t}-\bar{D}_{t}(\ln p) \geq$ $0, \forall n$. So $\bar{D}_{t}(\ln p) \leq \delta-g_{t}$, hence $p_{t} g_{t} \leq \delta p_{t}-\bar{D}_{t} p$ by claim 3 .

The part for $k_{t}>0$ is obvious, everything being differentiable there.

c: We have just shown that a5 implies the existence of a version of $r_{t}$ s.t. a4 holds. Conversely, assume such an $r$ : then $p_{t} g_{t} \leq \delta p_{t}-\bar{D}_{t} p$, with equality and $p_{t}$ differentiable when $k_{t}>0$, and we have to prove a5.

Since $p_{t}>0$, our inequality yields, by claim $3, g_{t} \leq \delta-\bar{D}_{t}(\ln p)=\underline{D}_{t} h$ with $h_{t}=\delta t-\ln p_{t}$ real-valued. Since $g \geq 0$, we first conclude that $h$ is increasing, from claim 2 , and then that $g \in L_{1}^{\text {loc }}$.

So we can define $\pi_{t}$ for each $t$ by the equation in a5, since $0<p_{t}<\infty$.

Constancy of $\pi$ on each interval where $k_{t}>0$ follows trivially from $p_{t} g_{t}=\delta p_{t}-p_{t}^{\prime}$, everything being differentiable there $(\bar{D} p=\underline{D} p)$.

If $f^{\prime}(0)<\infty$, then $g$ is bounded and continuous, so $\underline{D}_{t} \pi=\delta-g_{t}-\bar{D}_{t}(\ln p)$, $=\frac{1}{p_{t}}\left(\delta p_{t}-p_{t} g_{t}-\bar{D}_{t} p\right)$ by claim 3 , so $\underline{D}_{t} \pi \geq 0: \pi$ is increasing by claim 2 .

If $f^{\prime}(0)=\infty$, by continuity of $g,\{g>\delta\}$ is a disjoint union of open intervals. On each of those, we have $\bar{D}_{t} p \leq p_{t}\left(\delta-g_{t}\right)<0$, so $p_{t}$ and hence $\ln p_{t}=-\pi_{t}+\int_{0}^{t}\left(\delta-g_{s}\right) d s$ are decreasing; hence the singular part of that, which is the singular part of $-\pi_{t}$, is also decreasing. But $\pi$ is singular, since $\pi$ is constant on each of the open intervals where $g_{t}<\infty: \pi$ is carried by their closed complement $\{g=\infty\}$, which is negligible by local integrability of $g$. Thus $\pi$ itself is increasing on any such interval. Since it is also constant on each interval where $g<\infty$, it is locally increasing, thus increasing.

a6: The inequality follows from cor. 5. Local integrability follows from that of $\omega$ and $\zeta, p^{C}$ and $w$ being locally bounded.

d: If $K_{t}=0$ say for $t=0$, the fastest way the capital can increase afterwards is by having output equal to investment with full-employment in some interval after 0 ; the differential equation is then $k_{t}^{\prime}=\phi\left(k_{t}\right)$ with $\phi(k)=f(k)-R k$, hence, since $f(0)=0$ and $f^{\prime}(0)=\infty, g_{t}=\phi^{\prime}\left(k_{t}\right)$ is not locally integrable at 0 , by cor. 1 , and thus similarly is so, for any smaller $k_{t}$ : contradiction.

Comment 16. When $f^{\prime}(0)=\infty$, condition a5 is equivalent to the classical differential equation $\frac{d \ln p_{t}}{d t}=\delta-f^{\prime}\left(k_{t}\right)$, under the interpretation that the equality holds everywhere, and that derivatives may have $\overline{\mathbb{R}}$ values (but must be well-defined, so $p_{y}-p_{x}$ must be well-defined for $y$ sufficiently close to $x$, so that $p$ must be $\mathbb{R}$-valued to be differentiable). Indeed, the increasing aspect of $\pi_{t}$ ensures that the derivative is only decreased; but this happens only where $K_{t}=0$, i.e., $\frac{d \ln p_{t}}{d t}=-\infty$, so the equality is preserved: $p_{t}$ solves the differential equation. Conversely, any solution of the differential equation implies a $p_{t}$ as specified, using that a monotone function $H$ on $[0,1]$ is a.e. differentiable and $H_{1}-H_{0} \geq \int_{0}^{1} H_{t}^{\prime} d t$.

Corollary 9. Assume $p^{Y}=p^{I}$. If $f^{\prime}(0)=\infty$, then, a.e., $k_{t}>0$ and $w_{t}>0$.

Proof. Since $g_{t}=f^{\prime}\left(k_{t}\right) \in L_{1}^{\text {loc }}\left(\right.$ rem. 14), if $f^{\prime}(0)=\infty, k_{t}>0$ a.e. Hence $w_{t}>0$ a.e., because $f^{\prime}(0)=\infty$ implies $f(k)-k f^{\prime}(k)>0$ for $k>0$. 
Corollary 10. Assume $p^{Y}=p^{I}$. If $\omega_{x, s} \geq 0$ a.e. in $s$ and $f$ is not linear on $\left[0, k_{t}\right]$ for a.e. $t \in[x, x+1]$, then $M_{x}>0$. In particular, if $f(0)>0$ or $f^{\prime}(0)=\infty$, and $\omega \geq 0$ a.e., then $M_{x}>0$ a.e.

Proof. $w_{t}>0$ a.e. on $[x, x+1]$, by a3. In particular, if $f(0)>0, f$ is not linear on any interval $[0, k]$, while if $f^{\prime}(0)=\infty$, then $f$ is not linear on $\left[0, k_{t}\right]$ for $k_{t}>0$, and $k_{t}>0$ a.e. by cor. 9 .

3.6. Aggregate demand. Following-up on the conventions at the start of this section, note that for aggregate consumption the classic integration of correspondences $[1,7]$ does not apply, as consumption bundles are equivalence classes of arbitrary $\overline{\mathbb{R}}_{+}$-valued Lebesgue-measurable functions, so do not lie in any vector space. The modification required is described in App. A.

The specificity and strength of next lemma is that the integral of the demandcorrespondence, instead of being an arbitrary convex set, is the set of all measurable selections from a fixed correspondence $C$.

Lemma 11. Assume an equilibrium with $p^{C}$ locally bounded, and let

$$
C_{t}=N_{0}\left(e^{\beta t} p_{t}^{C}\right)^{-\sigma} \int_{t-1}^{t} e^{(\nu+\beta \sigma) x} \frac{M_{x}}{\int_{0}^{1}\left(p_{x+s}^{C}\right)^{1-\sigma} e^{-\beta \sigma s} d s} d x
$$

The integrand is a.e. well-defined, and the integral is finite, thus continuous in $t$. A.e., the integrand is null iff $M_{x}$ is so; thus $C_{t}>0$ except if $M_{x}=0$ a.e. on $[t-1, t]$.

If the right hand side is undefined, involving thus $\infty \times 0$, let $C_{t}=\overline{\mathbb{R}}_{+}$.

Then aggregate demand (the integral of individual demand) is the set of equivalence classes of all measurable selections from $C_{t}$.

Proof. Neglect all negligible sets of birthdates $x$ of cor. 6 , and take as domain $D$ the remaining part of $\mathbb{R}$. In particular, $M_{x}$ is everywhere well-defined on $D$ and $\in \mathbb{R}_{+}$, so lemma 4 is applicable, with $M_{x}$ for $M$ and $s \mapsto p_{x+s}^{C}$ for $p$, and demand is everywhere well-defined and non-empty, by lemma 4.a.

The demand correspondence $x \mapsto \Gamma(x)$ from $D$ to $\mathcal{M}^{\mathbb{R}}$ (cf. app. A), has a measurable graph, as the intersection of the following 3 measurable graphs: (a) $\{(x, c) \in$ $D \times \mathcal{M}^{\mathbb{R}} \mid c_{t}=0$ a.e. for $\left.t \notin[x, x+1]\right\}$, (b) $\left\{(x, c) \in D \times \mathcal{M}^{\mathbb{R}} \mid \int_{-\infty}^{\infty} p_{t}^{C} c_{t} d t \leq M_{x}\right\}$. (c) $\left\{(x, c) \in D \times \mathcal{M}^{\mathbb{R}} \mid U\left(s \mapsto c_{x+s}\right) \geq U_{x}^{*}\right\}$. Indeed, (a) is closed, measurability of (b) follows from that of $M_{x}$ (cor. 5) and the lower semi-continuity of $c \mapsto \int_{-\infty}^{\infty} p_{t}^{C} c_{t} d t$ (Fatou), and of (c) from that of $U^{*}$ (cor. 5), of $U$ on $\mathcal{M}^{[0,1]}$ (being by Fatou lower semi-continuous if $\sigma>1$ and else upper semi-continuous), and from the continuity of $(x, c) \mapsto\left(s \mapsto c_{x+s}\right): \mathbb{R} \times \mathcal{M}^{\mathbb{R}} \rightarrow \mathcal{M}^{[0,1]}$, which follows from the continuity of the convolution of the point mass at $-x$ and $h \circ c$ with $h$ a homeomorphism from $\overline{\mathbb{R}}$ to $[0,1]$ (see e.g., [19, prop. 2]) and of the projection from $\mathcal{M}^{\mathbb{R}}$ to $\mathcal{M}^{[0,1]}$.

Thus, the integral of $\Gamma$ is well-defined - recall we allow for correspondences to be defined only a.e., so equivalently, define, for $x \notin D$ where $\Gamma(x)$ is not defined ( $M_{x}$ being not defined, or $\left.\notin \mathbb{R}_{+}\right), \Gamma(x) \stackrel{\text { def }}{=} \mathcal{M}-$, and is the set of integrals over $x \in \mathbb{R}$ of all jointly measurable functions $c(x, t)$ s.t. $s \mapsto c(x, x+s) \in \Gamma(x) \forall x$.

Observe that requirement (a) was not part of our assumptions (cf. sect. 2.1.4), nor did we prove that in equilibrium no agent would buy any goods dated outside his life-span. But the same proof obviously shows that without this the demandcorrespondence is also measurable; we claim the integrals are the same, so our result is independent of any such assumption. Indeed, take a selection $c(x, t)$ as above from the larger correspondence, and define $\tilde{c}(x, t)=\mathbb{1}_{t-1 \leq x \leq t} c(x, t)+$ $\frac{1}{\Phi(-\nu)} \int_{y \notin[t-1, t]} e^{\nu(y-t)} c(y, t) d y$. Then clearly $\tilde{c}$ is measurable, has the same integral as $c$, and is a selection from the smaller correspondence: indeed, agents would have bought something at times $t$ outside their life-span only if $p_{t}^{C}=0$, since by lemma 8 (which applies since $p^{C}$ is locally bounded, and also by lemma 9.b, $\complement D$ is negligible) 
any increase in budget would increase their utility, so almost nobody's budget is affected by the change.

Lemma 4.c and 4.d imply then that the selection $c(x, t)$ must equal $c_{t-x}^{*}$ (ibidem) when either $U_{x}^{*} \in \mathbb{R}$ or $p_{t}^{C}>0$ and $M_{x}=0$. Since this is a measurable region, and $c_{t-x}^{*}$ is jointly measurable on this region, we can assume equality up to a (joint) null set, which does not affect the equivalence class of the integral. Further, using lemma 4.b, $U_{x}^{*} \in \mathbb{R} \equiv\left(\sigma>1 \vee M_{x}>0\right)$ since by cor. 3 and $5 U_{x}^{*}<\infty$ and since $p_{t}^{C}$ is locally bounded. Thus equality holds whenever $\sigma>1$ or $M_{x}>0$ or $p_{t}^{C}>0$, i.e., since the latter holds a.e. when $\sigma>1$ (cor.6.e), equivalently whenever $M_{x}>0$ or $p_{t}^{C}>0$.

Note that (1) follows at all $t$ s.t. $p_{t}^{C}>0$, by integration. And the integrand is a.e. well-defined, since $M_{x}<\infty$ a.e. (cor. 6.b) and since the denominator is a.e. $>0$ by cor. 6.d. A.e., it is null iff $M_{x}=0$ : if $M_{x}=0$, because the denominator is positive (cf. supra); and if $M_{x}>0$, because the denominator is finite, by local boundedness of $p^{C}$ if $\sigma<1$, and by cor. 6.a if $\sigma>1$. Thus the integral is always well-defined; so, $p^{C}$ being locally bounded, the right hand side is well-defined except where both the integral and $p_{t}^{C}$ are 0 , i.e., iff $M_{x}=0$ a.e. on $[t-1, t]$ and $p_{t}^{C}=0$, where it equals $\infty \times 0$.

Thus we show now equality in (1) when $p_{t}^{C}=0$ and $M_{x}$ is not negligible on $[t-1, t]$. As seen above, the right hand side is $\infty$ then, and, if $M_{x}>0, c(x, t)=c_{t-x}^{*}$, $=\infty$ (lemma 4 , finiteness of the denominator, since $M_{x}>0 \Rightarrow U^{*} \in \mathbb{R}$ ). Hence a non-negligible set of agents has infinite demand: aggregate demand is infinite too, thus equality.

For the remaining case, where $M_{x}=0$ a.e. on $[t-1, t]$ and $p_{t}^{C}=0$, i.e., the " $\infty \times 0$ " case, note $\sigma<1$ then, since else $p_{t}^{C}>0$ a.e. (cor.6.e). Almost all living agents have a null lifetime wealth; since they (almost) all face some non-negligible period in their lifetime where $p^{C}>0$ by cor. 6 .c, $U_{x}^{*}=-\infty$, so any consumption at times where $p_{t}^{C}=0$ is both feasible and optimal for them.

Since by lemma 9.a aggregate demand is locally integrable, it is a.e. finite, and thus so is the right hand side integral. Hence the integrand is locally integrable everywhere, and so the integral is everywhere finite, and is continuous in $t$.

\subsection{Avoiding the null equilbrium.}

Lemma 12. If either $C D$ is negligible and no labour is used in production $\forall t$ or $\omega \geq 0$ then $p_{t}^{Y}, p_{t}^{C}$ and $w_{t}$ are locally integrable. Assume further either $\sigma>1$ or $\exists t_{0}: f^{\prime}\left(k_{t}\right)>0$ a.e. on $\left[t_{0}, \infty\left[\right.\right.$. Then $p_{t}, p_{t}^{Y}$ are locally bounded away from 0 . If in addition $f(0)>0$ or $f^{\prime}(0)=\infty$, then also $w_{t}, Y_{t}>0$ a.e., and there is full-employment.

Proof. If $\omega \geq 0, M_{x}<\infty$ implies integrability of $w_{x+s} \zeta_{s}$. Else, let $\theta_{x, s}=$ $p_{x+s}^{C} \omega_{x, s}+w_{x+s} \zeta_{s}$ : $\complement D$ being negligible means that for a.e. $x$ either $\theta_{x, s}^{+}$or $\theta_{x, s}^{-}$ is integrable. $0 \leq M_{x}<\infty$ a.e. (cor. 5 and 6 .b) implies then that $\left|\theta_{x, s}\right|$ is integrable for a.e. $x$. Further, if no labour is supplied, the agent's budget constraint implies that both $p_{x+s}^{C} c_{x, s}$ and $w_{x+s} \zeta_{s}$ (spending on consumer goods and spending on leisure) are integrable, since both are non-negative and the sum of their integrals is $\leq M_{x}<\infty$. Integrability of $w_{x+s} \zeta_{s}$ for a.e. $x$ implies then by lemma 28 that $w_{t}$ is locally integrable, and $r_{t}$ is locally integrable by lemma 7 . By lemma 5.c $\mathfrak{c}\left(e^{-\gamma t} w_{t}, r_{t}\right) \geq p_{t}^{Y}$, and $\mathfrak{c}$ is concave and positively homogeneous, hence by Jensen's inequality $p_{t}^{Y}$ is locally integrable, and so is $p_{t}^{C}$ by lemma 5 .a. Thus either $\sigma>1$ or $\left(p^{C}\right)^{1-\sigma}$ is locally integrable. Hence, if either $\sigma>1$ or $\exists t_{0}: f^{\prime}\left(k_{t}\right)>0$ a.e. on $\left[t_{0}, \infty[\right.$, lemma 9.c implies that $p_{t}$ is locally bounded away from 0 , and so is $p_{t}^{Y}$ (lemma 5.a).

For the last point, lemma 7 implies $r_{t}$ is locally integrable and hence a.e. finite, so, by cor. $4, e^{-\gamma t} w_{t} \geq \sup _{k} g(k)$, with $g(k) \stackrel{\text { def }}{=} p_{t}^{Y} f(k)-r_{t} k$, and $\sup _{k} g(k)>0$ a.e. because $p_{t}^{Y}>0$ and $r_{t}$ is finite. Thus $w_{t}>0$ a.e. Therefore, by cor. 8 , there is full-employment. Since labour costs are $>0$, one must have $Y_{t}>0$. 
Lemma 13. Assume $f(0)=0, f^{\prime}(0)=\infty$. If $\omega \geq 0$, then $K_{t}$ is not identically 0 .

Proof. Else $f^{\prime}\left(k_{t}\right)>0 \forall t$, so $Y_{t}>0$ (lemma 12 ), so $K_{t}>0$ by $f(0)=0$.

\section{The Equilibrium EQuATions}

We start with a general characterisation of competitive equilibria of this economy and later focus on a special subclass of such equilibria, the balanced growth ones.

Definition 3. (a) Stationary endowments mean $\omega_{x, s}=e^{\gamma x} \omega_{s}$.

(b) A balanced growth equilibrium (BGE) is an equilibrium of an economy with stationary endowments, such that $K_{t}$ is an exponential function of time.

(c) A BGE is a golden rule equilibrium (GRE) if $\forall t, f\left(k_{t}\right)-R k_{t}=\max _{k}(f(k)-R k)$.

Notation 4.1. For stationary endowments, we use $E_{s}=\frac{e^{-(\gamma+\nu) s} \omega_{s}}{\int_{0}^{1} \zeta_{s} e^{-\nu s} d s}$ and $\Omega=\int E_{s} d s$.

Comment 17. Recall that in the basic model, zero-profits on the instantaneous production cone forces $p^{C}=p^{Y}=p^{I}$.

Theorem 1. The set of equilibria with $p^{C}=p^{Y}=p^{I}$ is the set of all solutions of the following system that respect the irreversibility constraints (i.e., the aggregate production $\left(Y_{t}-I_{t}, I_{t}, Y_{t}, L_{t}, K_{t}\right)$ belongs to the instantaneous production set):

(1) $Y_{t}=F\left(K_{t}, L_{t}\right)$

(2) $g_{t}=f^{\prime}\left(k_{t}\right), g$ is locally integrable

(3) $\quad p_{t}=e^{-\pi_{t}+\int_{0}^{t}\left(\delta-g_{s}\right) d s}, \quad p^{I}=p$

$0<p<\infty ; \pi_{t}$ is non-decreasing and constant on $t: K_{t}>0$

(4) $w_{t}=\left(f\left(k_{t}\right)-f^{\prime}\left(k_{t}\right) k_{t}\right) e^{\gamma t} p_{t}$, where $k f^{\prime}(k)$ is defined as 0 at 0 , by continuity.

(5) $C_{t}=\left(e^{\beta t} p_{t}\right)^{-\sigma} \int_{t-1}^{t} \frac{N_{0} e^{(\nu+\beta \sigma) x} M_{x}}{\int_{x+s}^{1-\sigma} e^{-\beta \sigma s} d s} d x$,

$M_{x} \stackrel{\text { def }}{=} \int_{0}^{1}\left(p_{x+s} \omega_{x, s}+w_{x+s} \zeta_{s}\right) d s \geq 0$ a.e.

(6) $I_{t}=N_{0} \int_{0}^{1} e^{\nu(t-s)} \omega_{t-s, s} d s+Y_{t}-C_{t}$

(7) $K_{t}=e^{-\delta t} \int_{-\infty}^{t} I_{s} e^{\delta s} d s$, with assumption 1

Individual consumption is then $\hat{c}_{x, s}=M_{x} \frac{e^{-\beta \sigma s}\left(p_{x+s}\right)^{-\sigma}}{\int_{0}^{1} e^{-\beta \sigma t}\left(p_{x+z}\right)^{1-\sigma} d z}$, and the rental rate of capital, $r_{t}$, must satisfy lemma 10.a4.

Proof. By lemma 7, $p^{I}=p$.

Start with equation (5). By lemma 10.a1, $p^{C}$ is locally bounded, so lemma 11 applies, and $p_{t}^{C}>0$ for all $t$, so aggregate consumption is a singleton. Hence, (5).

(7) comes from cor. 2 , and (6) is "market clearing," or the technology of the merchandising firms. Lemma 10 implies the rest (using also lemma 4 for $\hat{c}$ ).

Conversely, given a solution of those equations, lemma 10 implies the production sector maximises profits, $p_{t}$ is locally bounded and locally bounded away from 0 , and $M_{x}$ is a.e. well-defined, finite, and $\geq 0$. Hence, all agents have, by lemma 4.c,d, $\hat{c}_{x, s}$ as unique maximiser in their budget set, and those aggregate to $C_{t}$ by lemma 11.

Remark 18. It follows from the theorem that in the basic model (i.e., with full reversibility), for any equilibrium with $K_{t}>0 \forall t$, the price-system $p_{t}$ is $C^{1}$ and non-null, so its logarithm has a continuous derivative, $(\delta-) r_{t}$ in the terminology of [23]. Note that continuity of $r_{t}$ is the basic assumption of [23], so the conclusions 
of this classical paper (relied upon, implicitly or indirectly, everywhere in the literature) are, a posteriori, at least applicable in this case. In general, lemma 4, when re-written for general $u$, is needed - and much more powerful.

If one has $K_{t}>0 \forall t$ in the basic model, a direct argument leads to the conclusion, without relying on the full strength of our previous results: lemma 7 implies then $\ln p_{t}$ is $C^{1}, r_{t}$ being trivially determined since $K_{t}>0$, so [23] is applicable; this line of argument leads then directly to cor. 11 below.

Picking equilibria as in cor. 11, however, does not help to understand which equilibria are missed, if at all. Cor. 12 below gives a number of cases where none are missed; that, however, does rely on the full strength of our results.

Corollary 11. The set of equilibria where $K_{t}>0$ and $p^{C}=p^{Y}=p^{I}$, is the set of solutions (respecting the irreversibility constraints ${ }^{26}$ ) of the following system:

(a) $k \mapsto y: y_{t}=f\left(k_{t}\right)$

(b) $k \mapsto \mathfrak{r}: \mathfrak{r}_{t}=R-f^{\prime}\left(k_{t}\right)\left(=\gamma+\nu+\frac{p_{t}^{\prime}}{p_{t}}\right)$

(c) $(\mathfrak{r}, E) \mapsto \mathcal{N}_{1}: \mathcal{N}_{1, x}=\int_{0}^{1} e^{\int_{x}^{x+s} \mathfrak{r}_{t} d t} E_{x+s, s} d s$

(d) $(k, \mathfrak{r}) \mapsto \mathcal{N}_{2}: \mathcal{N}_{2, x}=\int_{0}^{1} e^{\int_{x}^{x+s} \mathfrak{r}_{t} d t} \varphi_{s}\left(f\left(k_{x+s}\right)-k_{x+s} f^{\prime}\left(k_{x+s}\right)\right) d s$

(e) $\left(\mathcal{N}_{1}, \mathcal{N}_{2}\right) \mapsto \mathcal{N}: \mathcal{N}=\mathcal{N}_{1}+\mathcal{N}_{2}, \mathcal{N} \geq 0$

(f) $\mathfrak{r} \mapsto \mathcal{D}: \mathcal{D}_{x}=\int_{0}^{1} e^{-\eta s+(1-\sigma) \int_{x}^{x+s}} \mathfrak{r}_{t} d t d s$

(g) $(\mathcal{N}, \mathcal{D}) \mapsto \mathcal{B}: \mathcal{B}=\frac{\mathcal{N}}{\mathcal{D}}$

(h) $(\mathfrak{r}, \mathcal{B}) \mapsto c: c_{t}=\int_{0}^{1} e^{-\eta u-\sigma \int_{t-u}^{t} \mathfrak{r}_{s} d s} \mathcal{B}_{t-u} d u$

(i) $(y, E, c) \mapsto i: i_{t}=y_{t}+\Omega_{t}-c_{t}$

(j) $i \mapsto \tilde{k}: \tilde{k}_{t}=e^{-R t} \int_{-\infty}^{t} e^{R s} i_{s} d s>0$, with assumption 1

The prices then can be computed as follows, using $g_{t} \stackrel{\text { def }}{=} f^{\prime}\left(k_{t}\right)$,

$$
\begin{aligned}
& p_{t}=p_{0} e^{\int_{0}^{t}\left(\delta-g_{s}\right) d s}, \quad p^{I}=p \\
& r_{t}=p_{t} g_{t} \\
& w_{t}=\left(f\left(k_{t}\right)-k_{t} f^{\prime}\left(k_{t}\right)\right) e^{\gamma t} p_{t}
\end{aligned}
$$

Proof. We start by showing that any equilibrium with $K_{t}>0$ and $p^{C}=p^{I}=p^{Y}$ has to satisfy conditions (a)-(j) and the price equations (1)-(3).

Using thm. $1, K_{t}>0$ implies $\pi_{t}$ is constant in the price equation, so eq. (1) holds with $p_{0}=e^{-\pi_{0}}$; eq. (2) is from lemma 10.a4 using $K_{t}>0$, eq. (3) is from thm. $1(4)$, because $k_{t}>0$.

Next, let $p_{x+s}=p_{x} \psi(x, s)$ with $\psi(x, s)=\exp \left(\delta s-\int_{x}^{x+s} f^{\prime}\left(k_{v}\right) d v\right)$. Now, substituting from thm. 1 ,

$$
\begin{aligned}
c_{t} & =\frac{C_{t}}{L_{t}}=\frac{\left(e^{\beta t} p_{t}\right)^{-\sigma}}{e^{(\gamma+\nu) t} \int_{0}^{1} \zeta_{s} e^{-\nu s} d s} \int_{t-1}^{t} e^{(\nu+\beta \sigma) x} \frac{\int_{0}^{1}\left(p_{x+s} \omega_{x, s}+w_{x+s} \zeta_{s}\right) d s}{\int_{0}^{1} p_{x+s}^{1-\sigma} e^{-\beta \sigma s} d s} d x \\
& =\int_{t-1}^{t} \frac{e^{(\nu+\beta \sigma)(x-t)-\gamma t}}{\int_{0}^{1} \zeta_{s} e^{-\nu s} d s} \frac{\int_{0}^{1} \psi(x, s)\left(\omega_{x, s}+e^{\gamma(x+s)}\left(y_{x+s}-f^{\prime}\left(k_{x+s}\right) k_{x+s}\right) \zeta_{s}\right) d s}{(\psi(x, t-x))^{\sigma} \int_{0}^{1}(\psi(x, s))^{1-\sigma} e^{-\beta \sigma s} d s} d x
\end{aligned}
$$

Use now $\omega_{x, s}=E_{x+s, s} e^{\gamma(x+s)+\nu s} \int_{0}^{1} \zeta_{u} e^{-\nu u} d u$ (cf. sect. 2.5), to re-write the numerator of the second ratio:

$$
\begin{aligned}
& \omega_{x, s}+\zeta_{s} e^{\gamma(x+s)}\left(y_{x+s}-f^{\prime}\left(k_{x+s}\right) k_{x+s}\right) \\
= & e^{\gamma(x+s)+\nu s}\left(E_{x+s, s} \int_{0}^{1} \zeta_{u} e^{-\nu u} d u+e^{-\nu s} \zeta_{s}\left(y_{x+s}-f^{\prime}\left(k_{x+s}\right) k_{x+s}\right)\right)
\end{aligned}
$$

\footnotetext{
${ }^{26}$ So that the aggregate production component $\left(Y_{t}-I_{t}, I_{t}, Y_{t}, L_{t}, K_{t}\right)$ of the solution belongs to the instantaneous production set.
} 


$$
\begin{aligned}
&=e^{\gamma(x+s)+\nu s}\left(\int_{0}^{1} \zeta_{u} e^{-\nu u} d u\right)\left(E_{x+s, s}+\varphi_{s}\left(y_{x+s}-f^{\prime}\left(k_{x+s}\right) k_{x+s}\right)\right) ; \text { so: } \\
& c_{t}=\int_{0}^{1} e^{\sigma\left(\int_{t-u}^{t} f^{\prime}\left(k_{s}\right) d s\right)-(\nu+\gamma+\sigma(\beta+\delta)) u} \mathcal{B}_{t-u} d u, \quad \text { where } \mathcal{B}_{x} \stackrel{\text { def }}{=} \frac{\mathcal{N}_{x}}{\mathcal{D}_{x}} \text { and: } \\
& \mathcal{N}_{x} \stackrel{\text { def }}{=} \int_{0}^{1} e^{R s-\int_{x}^{x+s} f^{\prime}\left(k_{v}\right) d v}\left(E_{x+s, s}+\varphi_{s}\left(y_{x+s}-f^{\prime}\left(k_{x+s}\right) k_{x+s}\right)\right) d s \\
& \mathcal{D}_{x} \stackrel{\text { def }}{=} \int_{0}^{1} e^{s(\delta-\sigma(\beta+\delta))-(1-\sigma)\left(\int_{x}^{x+s} f^{\prime}\left(k_{v}\right) d v\right)} d s
\end{aligned}
$$

Using the definition of $\eta(=(\gamma+\nu)(1-\sigma)+\beta \sigma)$ we obtain now (b)-(h). Conditions (a), (i) and (j) are from (1), (6) and (7) of thm. 1 respectively (with $K_{t}>0$ ).

Conversely, a solution of the system (a)-(j) along with the price equations (1)(3) and $p^{C}=p^{I}=p^{Y}$ is an equilibrium by thm. 1. Indeed, $k_{t}>0$ (from (j)) implies the local integrability of $g_{t}$, and hence, first (2) in thm. 1, and, second, $0<p_{t}<\infty$ by eq. 1 here, implying then (3) in thm. 1 with $\pi_{t}=-\ln p_{0}$. The equations for wages are identical. Conditions (1), (6) and (7) are from (a),(i) and (j) respectively. Finally, eq. 5 follows now by reversing the above computation.

\subsection{Implications of the equilibrium conditions.}

Corollary 12. Conditions a-j and 1-3 of corollary 11 characterise

(a) all equilibria of the general model, provided $\Omega_{t} \leq 0 \forall t$ (e.g., pure transfers), $f^{\prime}(0)=\infty$ and $f(0)=0$ and provided the production vector component $\left(Y_{t}-I_{t}, I_{t}, Y_{t}, L_{t}, K_{t}\right)$ of the solution belongs to the instantaneous production set for all $t$;

(b) if $f(0)=0, f^{\prime}(0)=\infty, \omega=0$, all equilibria of the general model in which constraints on disvestment are not binding (i.e., $p_{t}^{Y}=p_{t}^{I}$ ), provided the quantity component $\left(C_{t}, I_{t}, Y_{t}, L_{t}, K_{t}\right)$ of the solution belongs to the instantaneous production set for all $t$;

(c) all equilibria of the general model where $0<i_{t}<y_{t}$ a.e., provided the solution satisfies $0<i_{t}<y_{t}$;

(d) if $K_{t}$ is exponential, all BGE of the basic model, if $f^{\prime}(0)=\infty$;

(e) if $K_{t}$ is exponential, all BGE of the general model with $\omega=0, f(0)=0$ and $f^{\prime}(0)=\infty$.

Proof. By cor. 11, any solution to the system (a)-(j) is an equilibrium with $K>0$ and all prices equal. Remains to show that in each of the cases here an equilibrium has $K>0$ and all prices equal.

(a): by lemma 10.d.

(d): The equality of the prices is by lemma 5.b. Being exponential and bounded (prop. 1.a), $k_{t}$ is constant, positive by cor. 9 .

(b): Since $p^{Y}=p$ by lemma 7 , it follows from lemma 10.a1 that $p^{C}$ is locally bounded, hence lemma 11 applies. Cor. 10 implies that $M_{x}>0$ a.e., so equation (1) of lemma 11 holds everywhere, and with the integral and $C_{t}$ strictly positive, so $I_{t}<Y_{t}$ and all prices are equal $\left(p=p^{Y}=p^{I}=p^{C}\right)$.

Since the integral in the definition of aggregate demand (equation (1) of lemma 11 ) is locally bounded away from 0 , and since $p^{C}$ is locally bounded, $C_{t}$ itself is locally bounded away from 0 . Thus $K_{t}=0$ is impossible: immediately after such a time, it is impossible to have $C_{t}$ bounded away from 0 , if $f(0)=0$.

(c): We have $p^{C}=p^{Y}=p$ a.e., using lemma 5.b, hence thm. 1 applies. Since $i_{t}>0$ by the capital accumulation equation (thm. 1(7)), we have $k_{t}>0$.

(e): $K_{t}$ is exponential iff $k_{t}$ is, and by prop. 1.a, $k_{t}$ is bounded, so constant. Thus $K_{t}=K_{0} e^{(\gamma+\nu) t}$. Since $\omega \geq 0, K_{0}>0$ by lemma 13 . Then, by the capital 
accumulation equation, $I_{t}=R K_{t}$ is $>0$ too, so constraints on disvestment are not binding; so the rest follows by case $b$.

Comment 19. No Bubbles: To get rid of the solutions of the equation in lemma 7 with (even constant) $\varsigma>0$ ("bubbles"), or: indeterminacy) one might expect to need a transversality condition, e.g., $\lim _{t \rightarrow \infty} e^{-\delta t} p_{t}=0$, or infinitely-lived investment firms making arbitrage operations like buying some capital now and renting it out forever after. But thm. 1 (eq. (3)) does imply $\varsigma=0$, provided $f$ is strictly increasing: prop. 1.a implies $k_{t}$ is bounded, so inf $g_{t}=\inf f^{\prime}\left(k_{t}\right)>0$, thus $\int_{0}^{\infty} g_{t} d t=\infty$, and hence $\varsigma=\lim _{t \rightarrow \infty} e^{-\delta t} p_{t}=0$.

Comment 20. No Autarcy: The "intellectual reason" why the "0-equilibrium" (where $K_{t}=0 \forall t$ ) doesn't exist is individual rationality: a single Robinson Crusoe with no starting capital can produce output and capital and consumption goods in his lifetime (at least if $1 / f$ is locally integrable at 0 , cf. lemma 2 and comment 8 ), according to the maximal solution of lemma $2^{27}$ where he works full-time and all output is converted into investment. The problem with this "argument" is that if $\zeta$ is identically 0 in some initial part of his lifetime, capital (and hence consumption possibilities) will start to build up only after that initial segment, i.e., if $\sigma<1$, his lifetime utility is still $-\infty$ : that is why trading is needed with other Robinsons born at different dates, and hence the whole apparatus of equilibrium analysis.

Comment 21. Clearly, all the previous results apply to the reduced economy $\mathfrak{E}^{\prime}$ considered in section 2.5.3 as well. Since the map $\mathfrak{E}$ to $\mathfrak{E}^{\prime}$ is an isomorphism, it maps, in each direction, equilibria to equilibria.

Thus, under the assumptions of cor.11, in $\mathfrak{E}^{\prime}$, individual consumption equals $c_{x, s}^{\odot}=\mathcal{B}_{x} \exp \left(-\int_{x}^{x+s}\left(\sigma \mathfrak{r}_{t}+\eta\right) d t\right)$. Further, $p_{t}=p_{0} \exp \left(\int_{0}^{t} \mathfrak{r}_{s} d s\right)$ so that $-\mathfrak{r}_{t}$ is the equilibrium interest rate (net of depreciation) in $\mathfrak{E}^{\prime}$. Therefore, the map of the prices is then, with $P=\frac{p_{0}^{\prime}}{p_{0}}: p_{t} \mapsto p_{t}^{\prime}=P e^{(\gamma+\nu) t} p_{t}, w_{t} \mapsto w_{t}^{\prime}=P e^{\nu t} w_{t}, r \mapsto r^{\prime}=r$.

\section{Balanced Growth Equilibria}

It will be shown below that in a BGE where $k>0$ all quantities of the reduced economy are constant with time, and so will be $\mathfrak{r}=R-f^{\prime}(k)$. Finding the BGE amounts then to solving one market clearing equation for $\mathfrak{r}$, or simply $k$.

Notation 5.1. Let $\digamma(\mathfrak{r}) \stackrel{\text { def }}{=} \frac{\Phi(-\mathfrak{r} \sigma-\eta)}{\Phi(\mathfrak{r}(1-\sigma)-\eta)}$, and for a bounded measure $h$ on $[0,1]$ (or $\left.h \in L_{1}([0,1])\right)$ let $\tilde{H}_{h}(\mathfrak{r}) \stackrel{\text { def }}{=} \int h(d s)-\digamma(\mathfrak{r}) \int e^{\mathfrak{r} s} h(d s)$.

Remark 22. $\digamma(\mathfrak{r})$ decreases from $\infty$ to $\left(1-\sigma^{-1}\right)^{+} ; \digamma(0)=1$, so $\tilde{H}_{h}(0)=0$ for any bounded measure $h$ on $[0,1]$, and $\tilde{H}_{h}(\mathfrak{r}) / \mathfrak{r}$ is analytic (lemma 27$) . \tilde{H}$ is linear in $h$.

Corollary 13. The set of BGE satifying assumptions of cor. 11 is the set of constant positive solutions $k$ of the system

$$
\begin{aligned}
\int_{0}^{1} e^{\mathfrak{r} s} \Xi_{s} d s & \geq 0, \text { with } \Xi_{s} \stackrel{\text { def }}{=} E_{s}+\left(f(k)-k f^{\prime}(k)\right) \varphi_{s}, \text { and } \\
k \mathfrak{r} & =\tilde{H}_{\Xi}(\mathfrak{r}), \text { with } \mathfrak{r}=R-f^{\prime}(k)
\end{aligned}
$$

with the rest of the (constant) quantities determined by the following conditions:
(a) $\mathfrak{r}=R-f^{\prime}(k)$
(b) $y=f(k)$
(c) $i=R k$
(d) $\mathcal{N}=\int_{0}^{1} e^{\mathfrak{r} s} \Xi_{s} d s$
(e) $c=\digamma(\mathfrak{r}) \mathcal{N}$

\footnotetext{
${ }^{27}$ It is not unique, but it is feasible, and that is the only thing which matters.
} 
(f) $\mathcal{D}=\Phi(\mathfrak{r}(1-\sigma)-\eta), \mathcal{B}=\frac{\mathcal{N}}{\mathcal{D}}$

and the prices determined by

$$
\begin{aligned}
p_{t} & =p_{0} e^{(\mathfrak{r}-\gamma-\nu) t} \\
w_{t} & =\left(f(k)-k f^{\prime}(k)\right) e^{\gamma t} p_{t} \\
r_{t} & =p_{t} f^{\prime}(k)
\end{aligned}
$$

Remark 23. If $f^{\prime}(0)=\infty$, any non-negative solution $k$ of (2) is strictly positive. Hence cor. 12.d-e imply that BGE of those models are characterised by cor. 13 .

Proof. Being exponential and bounded (prop. 1.a), $k_{t}$ is constant, $=k$. By (a) in cor. $11, y_{t}=y$ is constant, and $(\mathrm{j})$ there implies $i_{t}=i$ is so. Since $\Omega_{t}=\Omega$ by definition of BGE, equation (i) implies $c_{t}=c$ is constant too.

For any fixed $\mathfrak{r}$, conditions (a)-(f) along with (1) of this corollary are equivalent to conditions (a)-(j) (excluding cond. (i)) of cor.11, for constant quantities. The equivalence of the corresponding price equations is obvious.

Next, cond. (i) of cor. 11 for constant quantities, i.e., $i=y+\Omega-c$, and (c) above imply $\Omega-c+y-R k=0$, so, by substituting the rest of the conditions,

$$
\Omega-\digamma(\mathfrak{r}) \int_{0}^{1} e^{\mathfrak{r} s} E_{s} d s+\left(f(k)-k f^{\prime}(k)\right)\left(1-\digamma(\mathfrak{r}) \int_{0}^{1} e^{\mathfrak{r} s} \varphi_{s} d s\right)=k \mathfrak{r}
$$

which is equivalent to (2), by notation 5.1 .

Remark 24. With $E \geq 0, \mathcal{N} \geq 0$ ((1) in cor. 13) is always satisfied; and for $E=0$, and $f(k)=A k^{\alpha}, A>0,0<\alpha<1$, equation (2) in cor. 13 can be represented as

$$
\mathfrak{r}\left(\frac{\tilde{H}_{\varphi}(\mathfrak{r})}{\mathfrak{r}} f^{\prime}(k)-\frac{\alpha}{1-\alpha}\right)=0, \text { with } k=\left[\frac{R-\mathfrak{r}}{A \alpha}\right]^{\frac{1}{\alpha-1}}
$$

Substituting for $k$ we get a single equation in $\mathfrak{r}$.

Remark 25. $\mathfrak{r}$ is the growth rate of aggregate values $\left(p_{t} C_{t}, p_{t} Y_{t}, p_{t} K_{t}\right)$ in the BGE, by eq. (3) in cor. 13, since aggregate quantities grow at rate $\gamma+\nu$.

5.1. Nonsensical (overinvestment) BGE. In this subsection assume $f^{\prime}(0)=\infty$ in the basic model. Hence in all BGE $k>0$ by cor. 12.d.

Produced consumption is positive, i.e., $y>i$, if and only if $k<\kappa$, where $\kappa>0$ satisfies $f(\kappa)=R \kappa$ (and so is uniquely defined by ass. 4 ).

Hence if $k \geq \kappa$ the whole production sector works for nothing, or even consumes part of the "manna" $\Omega$; no consumption good ever comes out of it.

Corollary 14. In the basic model, for any economy with $f^{\prime}(0)=\infty$, there is, for any $k>0$, a transfer policy $E_{s}$ such that $k$ is consistent with a BGE.

Proof. For the given economy take any value $k>0$. If $\mathfrak{r}=0$, pick any transfer policy that satisfies cor. 13.1 and use the fact that $\tilde{H}_{\Xi}(0)=0$ by rem. 22 to construct a BGE (using cor. 13). Else, pick $h \in L_{1}([0,1])$ such that $\int e^{s \mathfrak{r}} h(d s) \geq 0$ and $\tilde{H}_{h}(\mathfrak{r}) / \mathfrak{r}>0$ (otherwise there are $b_{1}, b_{2}$ not both zero, such that $b_{1} \tilde{H}_{h}(\mathfrak{r}) / \mathfrak{r}+b_{2} \int e^{s \mathfrak{r}} h(d s)=0$ for all $h$, which implies $\mathfrak{r}=0)$. For $y=f(k)$, let $z$ solve $z y \tilde{H}_{h}(\mathfrak{r})=k \mathfrak{r}$, then $z>0$, since $y>0$ and $\tilde{H}_{h}(\mathfrak{r}) / \mathfrak{r}>0$. Let $E_{s}=y z h_{s}-\left(y-k f^{\prime}(k)\right) \varphi_{s}$. Then $\Xi_{s}=E_{s}+(y-$ $\left.k f^{\prime}(k)\right) \varphi_{s}=y z h_{s}$, and by construction of $z$, and linearity of $\tilde{H}_{h}$ in $h$, the market clearing condition (2) of cor. 13 holds. Since $\int_{0}^{1} e^{\mathfrak{r} s} \Xi_{s} d s \geq 0$, (condition (1)) the initial $k$ along with the constructed transfers are consistent with a BGE by cor. 13 .

Remark 26. Since $y-i=c-\Omega$ and $c \geq 0$ ((i) and (e) of cor.11), a sufficient condition to avoid nonsensical equilibria is to set transfers such that $\Omega<0$ and that satisfy the equilibrium condition (1) of cor. 13, $\int_{0}^{1} e^{\mathfrak{r} s} \Xi_{s} d s \geq 0,($ assuring $c \geq 0$ ). Alternatively, one could use pure transfers, $\Omega=0$, and $c>0$. 


\subsection{Golden rule equilibria.}

Corollary 15. Assume $f^{\prime}(0)>R$ and the endowments are stationary.

(a) If $\Omega+\sup _{k \geq 0}(f(k)-R k) \geq 0$ then there exists a GRE.

(b) Denote GRE variables with superscript $G$. The GRE are the solutions of

(1) $f^{\prime}\left(k^{G}\right)=R$, so $\mathfrak{r}^{G}=0$

(2) $y^{G}=f\left(k^{G}\right)$

(3) $i^{G}=R k^{G}$

(4) $c^{G}=\Omega+f\left(k^{G}\right)-k^{G} f^{\prime}\left(k^{G}\right)=\mathcal{N}^{G}$

(5) $p_{t}^{G}=p_{0}^{G} e^{-(\gamma+\nu) t}, p^{I}=p^{C}=p^{Y}=p^{G}$

(6) $w_{t}^{G}=p_{0}^{G} e^{-\nu t}\left(y^{G}-R k^{G}\right), r_{t}^{G}=R p_{t}^{G}$

(7) $\mathcal{D}^{G}=\Phi(-\eta)$, where $\mathcal{N}, \mathcal{D}$ are defined by conditions (c) - (h) of cor. 11 .

(c) Inequality (a) is necessary for the existence of a feasible path, at least if $f$ is strictly concave.

Proof. (a): Since $f^{\prime}(0)>R$ and $f_{\infty}^{\prime}<R$, and $f$ is concave, and differentiable at $k>0$, there exists $k^{G}>0$ satisfying b1. Given $k^{G}$ define the rest of the variables from conditions b2-b7. Then the result follows by point $\mathrm{b}$.

(b): Take a solution of (b1)-(b7). Since $p^{I}=p^{Y}=p^{C}=p^{G}$, by thm. 1 it is an equilibrium. Since $k$ is constant, it is a BGE and it is a GRE by the concavity of $f$ and condition (b1).

Conversely, take an arbitrary GRE, then $k>0$ has to satisfy (b1). By the capital accumulation equation in section 2.2.2, (b3) has to hold. Then both $k$ and $i$ are strictly positive. Hence a constraint that investment can not be transformed into output is not binding, so, by lemma 5.b, $p^{I}=p^{Y}$, and thus lemma 10 applies. Its condition a2 implies (b2). This implies that $y>i$, since $y-i=f(k)-f^{\prime}(k) k>0$ for $k \geq k^{G}$, as $f^{\prime}(0)>R$ (indeed, $f(k)-f^{\prime}(k) k=0$ implies $f$ is linear on $[0, k]$, so $\left.f^{\prime}(k)=f^{\prime}(0)>R\right)$; so cor. 12.c applies. Thus, the GRE should solve the system a-j and the equations (1)-(3) of cor. 11, in addition to $p=p^{I}=p^{C}=p^{Y}$. It is easy to verify that conditions (b4)-(b7) here are implied by the first three conditions, (b1)-(b3) (which are consistent with (a), (b), (j) of cor. 11) and conditions (c)-(i) in cor. 11 along with its price equations.

The last part of the statement, (c), follows by the Pareto property of GRE [17, thm. 1] implying no feasible aggregate consumption is strictly higher than $c^{G}$.

5.3. Graphs of BGE without transfers for $\mathbf{f}(\mathbf{k})=\mathbf{A k}^{\alpha}$. We plot here eq. (1) of cor. 13, i.e., $\frac{\alpha}{1-\alpha}$ as a function of $x=1-\mathfrak{r} / R=f^{\prime}(k) / R=\alpha Y_{t} / I_{t}$. This way one can find the BGEs for any $\alpha$ by intersection with the corresponding horizontal line, i.e., solving eq. (1) of remark 24 in $x$ : $\frac{1-x}{x}\left(\frac{\tilde{H}_{\varphi}((1-x) R)}{1-x} x-\frac{\alpha}{1-\alpha}\right)=0$. With those coordinates, 1) the relevant region is the positive orthant, 2) units are dimensionless, thus easier to interpret, and 3) the function $\frac{\tilde{H}_{\varphi}(\mathfrak{r})}{\mathfrak{r}}$ is analytic (lemma 27), so the graph, more reliable.

Figures 1-4 show the BGE of economies with $\varphi(s)=\frac{1}{b-a} \mathbb{1}_{[a, b]}(s)$ and reasonable parameters (time unit being 1 lifetime).

One could make two observations based on the graphs. First, Gale's [12] dichotomy, the distinction between GRE and other BGE, is quite visible and it is made transparent in the next section using his classical net asset criterion. Second, the number of pure BGE can be either even or odd. Thm. 3 assures finiteness of BGE and provides simple conditions determining their parity.

\section{Gale's Dichotomy}

We first show that in any equilibrium the amount of net assets, the difference between total consumer savings and the debts of all firms, is constant over time. 


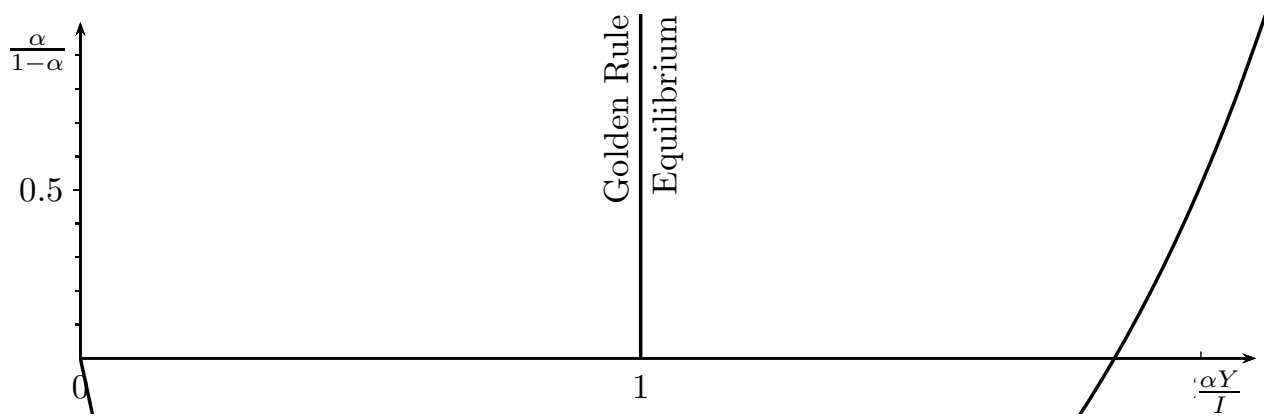

Figure 1. $R=11, \sigma=.5, \eta=2, a=.2, b=.75$. Two equilibria $\forall \alpha$.

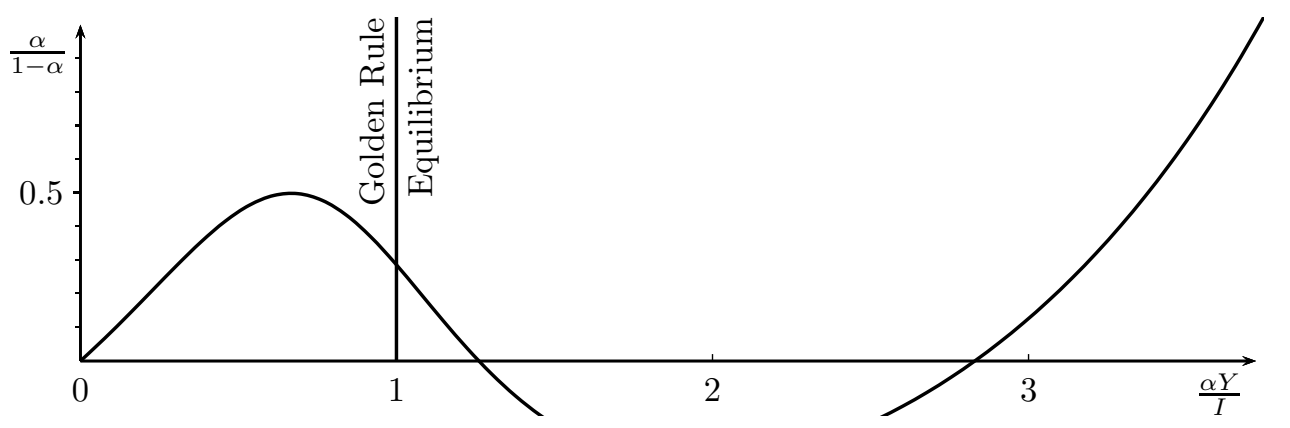

Figure 2. $R=11, \sigma=.25, \eta=2, a=.135, b=.5$. Two to four equilibria.

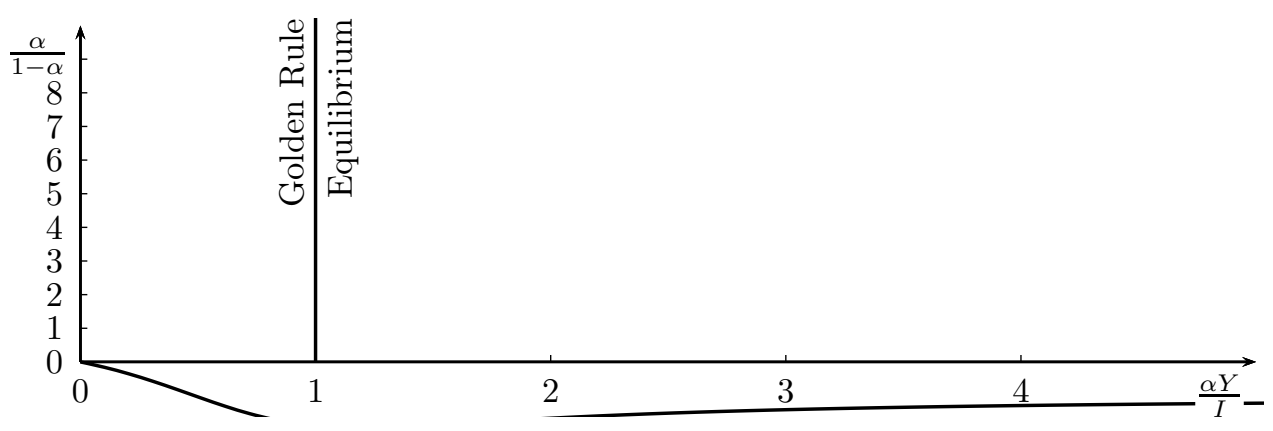

FiguRe 3. $R=10, \sigma=.25, \eta=2.5, a=.25, b=.75$. 1 equilibrium $\forall \alpha$.

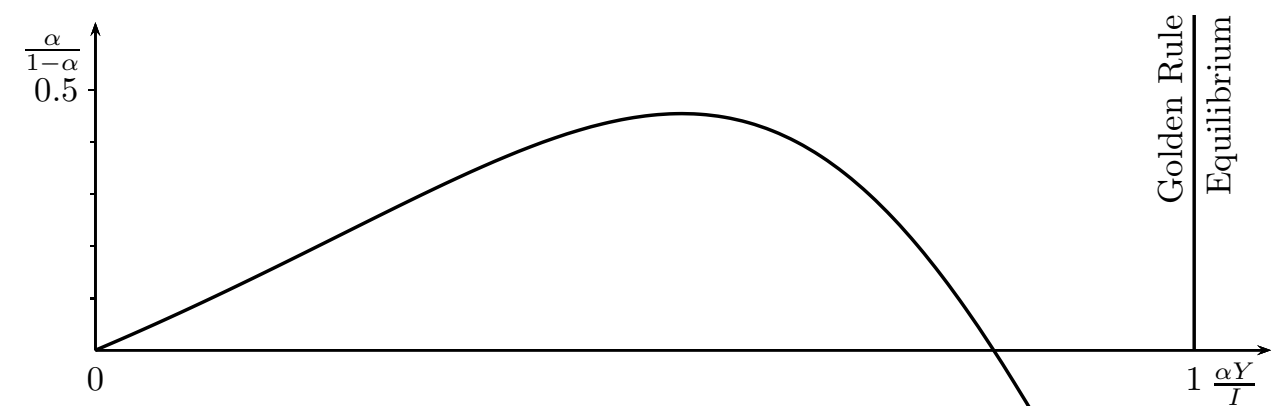

Figure $4 . R=15, \sigma=.24, \eta=1.9, a=.24, b=.55 .1$ or 3 equilibria. 
To give a meaningful definition of savings we will only consider equilibria where $p^{Y}=p^{I}$ and so, by lemma 10, the "instantaneous savings" of a consumer are locally integrable, and thus the total accumulated savings can be defined as a Lebesgue integral of the instantaneous ones, as we show in lemma 14.

Definition 4. For any equilibrium with $p^{Y}=p^{I}$ let savings $S_{t} \stackrel{\text { def }}{=} \int_{t-1}^{t} \int_{0}^{t-x} h_{x+s, s} d s d x$, where $\left.h_{z, s} \stackrel{\text { def }}{=} N_{0} e^{\nu(z-s)} p_{z}^{C}\left(\omega_{z-s, s}-\hat{c}_{z-s, s}\right)+w_{z} \zeta_{s}\right)$ denotes the instantaneous savings of an individual who is of age $s$ at time $z$; and let net assets ${ }^{28} m_{t}=S_{t}-p_{t} K_{t}$.

Remark 27. By lemma 10 consumers work full-time and spend all of their income, so $S_{t}$ denotes their actual savings. $p_{t} K_{t}$ is the total outstanding debt of all firms; indeed, all firms but investment firms are instantaneous, so their debt is zero. Any investment firm $f$ has a finite life-span and its starting value is zero. So by the zero-profit condition its value at any time $t$ is also zero, hence its debt at time $t$ equals the value of its capital, $p_{t} K_{t}^{f}$.

Lemma 14. $h_{z, s}=N_{0}\left[p_{z}^{C} e^{\nu(z-s)}\left(\omega_{z-s, s}-\hat{c}_{z-s, s}\right)+p_{z}\left(f\left(k_{z}\right)-k_{z} f^{\prime}\left(k_{z}\right)\right) e^{(\gamma+\nu) z} e^{-\nu s} \zeta_{s}\right]$ is locally Lebesgue integrable, so $S$ and $m$ are well-defined.

Proof. By lemma 10.a3, $w_{z}=\left(f\left(k_{z}\right)-k_{z} f^{\prime}\left(k_{z}\right)\right) e^{\gamma z} p_{z}$. By lemma 10.a1, $p_{z}$ is locally bounded, by prop.1.a, $f\left(k_{z}\right)$ is locally bounded, and since by lemma 5.c, $w$ is non-negative, and also $k_{z} f^{\prime}\left(k_{z}\right) \geq 0, w$ is locally bounded. Hence, since $\zeta$ is integrable, $w_{z} \zeta_{s}$ is locally integrable (in $z, s$ ). Next, $p^{C}$ is locally bounded by lemma 10.a1, $\omega$ is locally integrable by assumption (section 2.1.3), and individual consumption $\left(\hat{c}_{x, s}\right)$ is locally integrable by prop. 1.c. It follows that $h$ is locally (Lebesgue-)integrable.

Theorem 2. $m_{t}=m$ is constant in any equilibrium where $p^{Y}=p^{I}$.

Proof. By lemma 14, $h$ is locally integrable, so, by Fubini, $S_{t}=\int_{0}^{1} \int_{t-1+s}^{t} h(z, s) d z d s$. Since for any $a, b, c, d \in \mathbb{R}, \int_{a}^{b}-\int_{c}^{d}=\int_{a}^{c}-\int_{b}^{d}$,

$$
\begin{aligned}
S_{b}-S_{a} & =\int_{0}^{1}\left[\int_{a}^{b} h_{z, s} d z-\int_{a-1+s}^{b-1+s} h_{z, s} d z\right] d s \\
& =\int_{a}^{b} \int_{0}^{1} h_{z, s} d s d z-\int_{a}^{b} \int_{0}^{1} h_{x-1+s, s} d s d x=\int_{a}^{b} \int_{0}^{1} h_{z, s} d s d z
\end{aligned}
$$

where the second equality holds because $\int_{0}^{1} h_{x-1+s, s} d s$, being the total life-time savings of the individuals born at $x-1$, is zero. It follows that $S$ is the primitive (in the sense of Lebesgue integral) of $\int_{0}^{1} h_{t, s} d s$, its a.e. derivative, $S_{t}^{\prime}$.

Thus, using the definition of $L_{t}$, the notation in sect. 2.5.1 $\left(\int_{0}^{1} N_{0} e^{t-s} \omega_{t-s, s} d s=\right.$ $\left.\Omega_{t} L_{t}, \int_{0}^{1} N_{0} e^{t-s} \hat{c}_{t-s, s} d s=c_{t} L_{t}\right)$ and the formula for $h$ in lemma 14, for a.e. $t$

$$
S_{t}^{\prime}=\int_{0}^{1} h_{t, s} d s=L_{t}\left(p_{t}^{C}\left(\Omega_{t}-c_{t}\right)+p_{t}\left(f\left(k_{t}\right)-f^{\prime}\left(k_{t}\right) k_{t}\right)\right)
$$

Next we show that the firms' debt, $p_{t} K_{t}$, is locally absolutely continuous.

Take an interval $[a, b]$, let $T_{0}$ be its subset where $K$ is zero. Pick $\epsilon>0$. By continuity of $K$ there is $\delta_{0}>0$ such that for any $t$ which is at least $\delta_{0}$-close to $T_{0}$, $K_{t}<\frac{\epsilon}{2 V(p)}$, where $V(p)$ is the variation of $p$ in $[a, b] . V(p)<\infty$ by lemma 10.a5 and by rem. 14 .

Let $Q$ be the closed $\frac{\delta_{0}}{2}$-neighbourhood of $T_{0}$.If $Q$ is non-empty, it consists of nonoverlapping closed intervals of length of at least $\delta_{0} / 2>0$. There can be at most

\footnotetext{
${ }^{28}$ Or money, foreign credit.
} 
a finite number $(n)$ of those intervals in $[a, b]$. Consider the complement of $Q$ in ]$a, b[$, it is a union of at most $n+1$ disjoint open intervals. Let $Z$ be the set of those intervals. Since $K_{t}>0$ on the closure of any $z \in Z$ (being at least $\delta_{0} / 2$ away from the closest zero), $p_{t} K_{t}$ is absolutely continuous there, so for any $z$, and any finite collection of non-overlapping intervals $\left[a_{j}, b_{j}\right]_{j}$ in the closure of $z$ there is $\delta_{z}>0$ such that $\sum_{j}\left|p_{a_{j}} K_{a_{j}}-p_{b_{j}} K_{b_{j}}\right|<\frac{\epsilon}{4(n+1)}$ whenever $\sum_{j}\left|a_{j}-b_{j}\right|<\delta_{z}$. Let $\delta_{Z}=\min _{z} \delta_{z}$. Then for any finite collection of non-overlapping intervals in the closure of $[a, b] \backslash Q$,

$$
\sum_{j}\left|a_{j}-b_{j}\right|<\delta_{Z} \Rightarrow \sum_{j}\left|p_{a_{j}} K_{a_{j}}-p_{b_{j}} K_{b_{j}}\right|<\frac{\epsilon}{4}
$$

Choose any $\delta \in] 0, \min \left\{\delta_{0} / 2, \delta_{Z}\right\}[$. Then any finite collection of non-overlapping intervals of total length less than $\delta$ can be partitioned into two groups: those that intersect $Q\left(\left[a_{i}, b_{i}\right]_{i}\right)$; and those that do not $\left(\left[a_{j}, b_{j}\right]_{j}\right.$, considered above). Since $\delta<\delta_{0} / 2$, the intervals of the first group are in the closed $\delta_{0}$-neighbourhood of $T_{0}$, and hence $B \stackrel{\text { def }}{=} \sum_{i}\left|K_{b_{i}}\right|\left|p_{a_{i}}-p_{b_{i}}\right|<\frac{\epsilon}{2 V(p)} V(p)=\frac{\epsilon}{2}$. Since by cor. $2 K_{t}$ is absolutely continuous, there is $\delta_{k}>0$ such that whenever $\sum_{i}\left|a_{i}-b_{i}\right|<\delta_{k}, \sum_{i}\left|K_{a_{i}}-K_{b_{i}}\right|<\frac{\epsilon}{4 P}$, where $P<\infty$ is the supremum of $p$ in $[a, b]$. Thus, $A \stackrel{\text { def }}{=} \Sigma_{i}\left|p_{a_{i}}\right|\left|K_{a_{i}}-K_{b_{i}}\right|<$ $P \frac{\epsilon}{4 P}=\frac{\epsilon}{4}$. Hence if $\delta$ is also below $\delta_{k}$, we have $\sum_{i}\left|p_{a_{i}} K_{a_{i}}-p_{b_{i}} K_{b_{i}}\right| \leq A+B=\frac{3}{4} \epsilon$. Combining with eq. (1) we get the absolute continuity of $p_{t} K_{t}$ on $[a, b]$.

Since $S_{t}$ is absolutely continuous, being a Lebesgue primitive, their difference, $m_{t}$, is absolutely continuous too.

Next, by lemma 10.a5 $p_{t} L_{t}=q_{t} L_{0}$ with $q_{t} \stackrel{\text { def }}{=} e^{-\pi_{t}+\int_{0}^{t}\left(R-f^{\prime}\left(k_{z}\right)\right) d z}$. So $p_{t} K_{t}=$ $L_{0} q_{t} k_{t}$. Since $q_{t} k_{t}$ is the product of two functions that are a.e. differentiable, the derivative of the firms' debt is

$$
L_{0}\left(q_{t}\left(R-f^{\prime}\left(k_{t}\right)-\pi_{t}^{\prime}\right) k_{t}+q_{t}\left[i_{t}-R k_{t}\right]\right)=L_{0} q_{t}\left[-f^{\prime}\left(k_{t}\right) k_{t}+i_{t}\right]
$$

where the last equality holds since $\pi_{t}^{\prime} k_{t}=0$ by lemma 10.a5.

Hence, for a.e. $t$, the difference, $m_{t}^{\prime}$, of the two derivatives, $S_{t}^{\prime}$ and $\left(p_{t} K_{t}\right)^{\prime}$, is $p_{t}^{C} L_{t}\left(\Omega_{t}-c_{t}\right)+L_{0} q_{t}\left(f\left(k_{t}\right)-i_{t}\right)$. If $p_{t}^{C}<p_{t}$, then no output is transformed into consumption, and no endowment is transformed into output, hence output equals investment, so the last summand is zero. The only source of consumption is the endowment, so the first summand is zero as well. Otherwise, by lemma $5 . \mathrm{a}, p_{t}^{C}=p_{t}$, then $m_{t}^{\prime}=L_{0} q_{t}\left[\Omega_{t}-c_{t}+f\left(k_{t}\right)-i_{t}\right]$, which is 0 by material balance (feasibility). Thus, $m_{t}^{\prime}=0$ a.e. Since $m_{t}$ is absolutely continuous, it is constant.

Comment 28. Consider all transactions as being paid through individual- or firmaccounts at a single bank, in the numeraire underlying our price system $p_{t}$ (so an interest-free money). Think of all those payments being made on the date of the corresponding physical transfer of goods, and of each account's balance as a function of time. Budget balance implies that only the accounts of currently living consumers or investment firms have a non-zero balance. So the total credit $m_{t}$ extended by this bank at time $t$ is the sum of the balances of all currently living agents. But since any transaction credits one account by the same amount it debits another one, $m_{t}$ is constant over time in equilibrium.

Now we give a necessary condition for $m$ to be non-zero, but only for BGE. 
Corollary 16. In any $B G E$ where $k_{t}>0$ and $p^{I}=p^{C}=p^{Y},{ }^{29}$

either (it is a "pure BGE"):

$$
m=0, \quad \text { i.e., } \quad k=\frac{\tilde{H}_{\Xi}(\mathfrak{r})}{\mathfrak{r}}
$$

or (it is a GRE):

$$
\mathfrak{r}=0, \quad \text { so, } \quad m=L_{0} p_{0}\left(c^{G}\left(\frac{1}{\eta}-\frac{1}{e^{\eta}-1}\right)-\int_{0}^{1} s E_{s} d s+\left(\Omega-c^{G}\right) \int_{0}^{1} s \varphi_{s} d s-k^{G}\right)
$$

where $\tilde{H}$ is as defined in notation 5.1 and $\Xi$ is as defined in cor. 13 .

Proof. By cor. 15 a BGE with $\mathfrak{r}=0$ is a GRE. $\frac{\tilde{H} \Xi(\mathfrak{r})}{\mathfrak{r}}$ is well-defined for all $\mathfrak{r}$ by lemma 27. By cor. $13 k, y$ are constant, and $E_{t, s}=E_{s}, c_{t, s}^{\odot}=c_{s}^{\odot}$. By cor. 13.3, $L_{t} p_{t}=p_{0} L_{0} e^{\mathfrak{r} t}$. Then, by the def. 4 , and using lemma 14 along with the definition of $\Xi_{s} \stackrel{\text { def }}{=} E_{s}+\left(f(k)-k f^{\prime}(k)\right) \varphi_{s}$,

$$
m=p_{0} L_{0}\left[\int_{t-1}^{t} \int_{0}^{t-x} e^{\mathfrak{r}(x+s)}\left[\Xi_{s}-c_{s}^{\odot}\right] d s d x-e^{\mathfrak{r} t} k_{t}\right]
$$

By lemma 14 the integrand is locally integrable, so by Fubini, the first summand in the brackets is $\int_{0}^{1} \int_{t-1}^{t-s} e^{\mathfrak{r}(x+s)}\left[\Xi_{s}-c_{s}^{\odot}\right] d x d s$ and if $\mathfrak{r} \neq 0$ it becomes $\frac{e^{\mathfrak{r} t}}{\mathfrak{r}} \int_{0}^{1}(1-$ $\left.e^{\mathfrak{r} s-\mathfrak{r}}\right)\left[\Xi_{s}-c_{s}^{\odot}\right] d s$. Since $\int_{0}^{1} e^{\mathfrak{r} s}\left[\Xi_{s}-c_{s}^{\odot}\right] d s=0$ (the value of accumulated savings is zero at death), this term simplifies to

$$
\frac{e^{\mathfrak{r} t}}{\mathfrak{r}} \int_{0}^{1}\left[\Xi_{s}-c_{s}^{\odot}\right] d s=\frac{e^{\mathfrak{r} t}}{\mathfrak{r}}\left(\int_{0}^{1} \Xi_{s} d s-c\right)
$$

where by sect. 2.5.2, $c=\int_{0}^{1} c_{s}^{\odot} d s$ and by cor. 13.e, $c=\digamma(\mathfrak{r}) \int_{0}^{1} e^{\mathfrak{r} s} \Xi_{x} d s$.

So, for the case $\mathfrak{r} \neq 0$, using the definition of $\tilde{H}_{h}$, notation 5.1 , we get

$$
m=L_{0} p_{0} e^{\mathfrak{r} t}\left(\frac{\tilde{H}_{\Xi}(\mathfrak{r})}{\mathfrak{r}}-k\right)=0
$$

where the last equality is by cor. 13.2.

Remains to deal with the case $\mathfrak{r}=0$ (GRE). The double integral in equation (1) is simply $\int_{0}^{1}(1-s)\left[\Xi_{s}-c_{s}^{\odot}\right] d s$. Next, using also comment $21, c_{x+s, s}^{\odot}=c_{s}^{\odot}=$ $\mathcal{B} e^{-s(\sigma \mathfrak{r}+\eta)}$. By cor. $15, \mathcal{N}^{G}=c^{G}$ and $\mathcal{D}^{G}=\Phi(-\eta)$, so $c_{s}^{\odot}=\frac{e^{-\eta s}}{\Phi(-\eta)} c^{G}$. Substituting this into eq. (1), and then using material balance, $\int_{0}^{1} \Xi_{s}^{G} d s=c^{G}$,

$$
\begin{aligned}
m & =L_{0} p_{0}\left(\int_{0}^{1}(1-s)\left[\Xi_{s}^{G}-\frac{e^{-\eta s}}{\Phi(-\eta)} c^{G}\right] d s-k^{G}\right) \\
& =L_{0} p_{0}\left(\frac{c^{G}}{\Phi(-\eta)}\left(\frac{1}{-\eta} e^{-\eta}-\int_{0}^{1} \frac{1}{-\eta} e^{-\eta s} d s\right)-\int_{0}^{1} s \Xi_{s}^{G} d s-k^{G}\right) \\
& =L_{0} p_{0}\left(c^{G}\left(\frac{1}{\eta}-\frac{1}{e^{\eta}-1}\right)-\int_{0}^{1} s E_{s} d s+\left(\Omega-c^{G}\right) \int_{0}^{1} s \varphi_{s} d s-k^{G}\right)
\end{aligned}
$$

Remark 29. With zero transfers, the first case yields the curve in fig. 1-4, and the second, the vertical.

Remark 30. The two cases described in cor. 16 correspond to Gale's (1973) dichotomy between "balanced" (where $m=0$ ) and "golden rule" equilibria (where $m$ is typically non-zero). Also, whether $m$ in the second alternative is positive or negative determines whether the model is "Samuelson" or "classical" in his terminology. 30

\footnotetext{
${ }^{29}$ In particular, in any BGE of the models in cor. 12.d-e, see remark 23.

${ }^{30}$ In our graphs, values of $\alpha$ corresponding to a point on the GRE vertical lying above (below) the curve correspond to a "classical" ("Samuelson") model.
} 


\section{Finiteness and Parity of BGe}

Notation 7.1. For $h \in L_{1}(\mathbb{R}), a_{h} \stackrel{\text { def }}{=} \inf \operatorname{Supp}\left(h_{t} d t\right) \in \overline{\mathbb{R}}\left(=\inf \left\{x \mid \int_{-\infty}^{x} h_{t} d t \neq 0\right\}\right)$.

Definition 5. $q: \mathbb{R} \rightarrow \mathbb{R}$ converges exponentially at rate almost $z$ (to a limit) if it converges exponentially at rate higher than $z-\varepsilon$ for any $\varepsilon>0$, and not at rate $z$.

Lemma 15. Assume $f^{\prime}(0)=\infty$. For $h \in L_{1}([0,1])$

(a) If $a_{h} \geq \sigma, \lim _{k \rightarrow 0} \tilde{H}_{h}\left(R-f^{\prime}(k)\right)=\int_{0}^{1} h_{s} d s$. The convergence is exponential in $\mathfrak{r}=R-f^{\prime}(k)$ if $a_{h}>\sigma$.

(b) If $a_{h}<\sigma$ and if there is $b>a_{h}$ such that, a.e. on $\left[a_{h}, b\right], h$ does not change sign, then $\lim _{k \rightarrow 0} \tilde{H}_{h}\left(R-f^{\prime}(k)\right)$ equals $-\infty$ if that sign is positive and $+\infty$ otherwise. The convergence is exponential in $\mathfrak{r}$ at rate almost $\min \{\sigma, 1\}-a_{h}$.

Proof. Since $f^{\prime}(0)=\infty$, by definition of $\tilde{H}_{h}$ (notation 5.1), its limit as $k \rightarrow 0$ is $\lim _{\mathfrak{r} \rightarrow-\infty}\left(\int h_{s} d s-\digamma(\mathfrak{r}) \int_{a_{h}}^{1} e^{\mathfrak{r} s} h_{s} d s\right)=\int_{0}^{1} h_{s} d s-\lim _{\mathfrak{r} \rightarrow-\infty} \digamma(\mathfrak{r}) \int_{a_{h}}^{1} e^{\mathfrak{r} s} h_{s} d s$.

(a): The statement is equivalent to $\lim _{\mathfrak{r} \rightarrow-\infty} \digamma(\mathfrak{r}) \int_{a}^{1} e^{\mathfrak{r} s} h_{s} d s=0$, with exponential convergence. Hence, first, by the dominated convergence theorem it suffices to show, that, for $\sigma<s<1, \digamma(\mathfrak{r}) e^{\mathfrak{r} s}$ is bounded uniformly in $(\mathfrak{r}, s)$ and converges a.e. to 0 as $\mathfrak{r} \rightarrow-\infty$. $\digamma(\mathfrak{r}) e^{\mathfrak{r} s}=-e^{\mathfrak{r}(s-\sigma)-\eta} \frac{1-e^{\mathfrak{r} \sigma+\eta}}{1-e^{\mathfrak{r}(1-\sigma)-\eta}} \frac{\mathfrak{r}(1-\sigma)-\eta}{-\mathfrak{r} \sigma-\eta}$. The last ratio is bounded by $\left|1-\frac{1}{\sigma}\right|+1$ for $\mathfrak{r}$ sufficiently small and the middle ratio is bounded by 2. The first term is bounded, $\left|e^{\mathfrak{r}(s-\sigma)-\eta}\right| \leq e^{-\eta}$ for $\mathfrak{r} \leq 0$ and converges to 0 .

Convergence is exponential, since, by a similar calculation, $\lim _{\mathfrak{r} \rightarrow-\infty} \digamma(\mathfrak{r}) \int_{a_{h}}^{1} e^{s \mathfrak{r}} h_{s} d s$ $\leq \frac{1-\sigma}{\sigma} \lim _{\mathfrak{r} \rightarrow-\infty} e^{\left(a_{h}-\sigma\right) \mathfrak{r}} g_{h}$, where $g_{h} \stackrel{\text { def }}{=} \int_{a_{h}}^{a_{h}+\varepsilon}\left|h_{s}\right| d s$ for some $\varepsilon>0$.

(b): It suffices to prove that if $h$ is positive on $\left[a_{h}, b\right]$ then $\lim _{\mathfrak{r} \rightarrow-\infty} \digamma(\mathfrak{r}) \int_{a_{h}}^{1} e^{\mathfrak{r} s} h_{s} d s$ $=+\infty$, since if $h$ is negative on $\left[a_{h}, b\right]$, one can use the same argument for $-h$.

Choose $b<\min \{\sigma, 1\}$, and take $v: a<v<b$. Since $\mathfrak{r} \leq 0$, shifting the whole mass $Z_{v} \stackrel{\text { def }}{=} \int_{0}^{v} h_{s} d s>0$ on $[a, v]$ to $\{v\}$ yields $\int_{0}^{v} e^{s \mathfrak{r}} h(s) d s \geq e^{v \mathfrak{r}} Z_{v}$. And, since $h_{s}$ is positive on $[v, b]$, for sufficiently small $\mathfrak{r} \leq 0, \int_{v}^{1} e^{\mathfrak{r} s} h_{s} \bar{d} s \geq 0$ and so $\int_{a_{h}}^{1} e^{\mathfrak{r} s} h_{s} d s \geq e^{\mathfrak{r} v} Z_{v}$.

Next, if $\sigma<1, \digamma(\mathfrak{r})=-e^{-\mathfrak{r} \sigma-\eta} \frac{\mathfrak{r}(1-\sigma)-\eta}{-\mathfrak{r} \sigma-\eta} \frac{1-e^{\mathfrak{r} \sigma+\eta}}{1-e^{\mathfrak{r}(1-\sigma)-\eta}}$. The last ratio converges to 1 , and $\frac{\mathfrak{r}(1-\sigma)-\eta}{-\mathfrak{r} \sigma-\eta} \rightarrow-\frac{1-\sigma}{\sigma}$. Hence as $v<\sigma$,

$$
\lim _{\mathfrak{r} \rightarrow-\infty} \digamma(\mathfrak{r}) \int_{a_{h}}^{1} e^{s \mathfrak{r}} h_{s} d s \geq Z_{v} \frac{1-\sigma}{\sigma} \lim _{\mathfrak{r} \rightarrow-\infty} e^{\mathfrak{r}(v-\sigma)-\eta}=+\infty
$$

Similarly $\digamma(\mathfrak{r})=e^{-\mathfrak{r}} \frac{\mathfrak{r}(1-\sigma)-\eta}{-\mathfrak{r} \sigma-\eta} \frac{1-e^{\mathfrak{r} \sigma+\eta}}{1-e^{\mathfrak{r}(\sigma-1)+\eta}}$, so, if $\sigma>1$,

$$
\lim _{\mathfrak{r} \rightarrow-\infty} \digamma(\mathfrak{r}) \int_{a_{h}}^{1} e^{s \mathfrak{r}} h_{s} d s \geq Z_{v} \frac{\sigma-1}{\sigma} \lim _{\mathfrak{r} \rightarrow-\infty} e^{(v-1) \mathfrak{r}}=+\infty
$$

For the rate of convergence note that the above inequalities hold for $v$ arbitrarily close to $a_{h}$ (only $Z_{v}>0$ is needed). On the other hand, $\int_{a_{h}}^{v} e^{s \mathfrak{r}} h(s) d s \leq e^{a_{h} \mathfrak{r}} Z_{v}$; so, for sufficiently low $\mathfrak{r} \leq 0, \int_{a_{h}}^{1} e^{s \mathfrak{r}} h(s) d s \leq 2 e^{a_{h} \mathfrak{r}} Z_{b}$. Thus:

$$
\begin{array}{ll}
\lim _{\mathfrak{r} \rightarrow-\infty} \digamma(\mathfrak{r}) \int_{a_{h}}^{1} e^{s \mathfrak{r}} h_{s} d s \leq 2 Z_{b} \frac{1-\sigma}{\sigma} \lim _{\mathfrak{r} \rightarrow-\infty} e^{\mathfrak{r}\left(a_{h}-\sigma\right)-\eta}, & \text { if } \sigma<1 \\
\lim _{\mathfrak{r} \rightarrow-\infty} \digamma(\mathfrak{r}) \int_{a_{h}}^{1} e^{s \mathfrak{r}} h_{s} d s \leq 2 Z_{b} \frac{\sigma-1}{\sigma} \lim _{\mathfrak{r} \rightarrow-\infty} e^{\left(a_{h}-1\right) \mathfrak{r}}, & \text { if } \sigma>1
\end{array}
$$

Assumption 5. $\lim _{k \rightarrow 0} \frac{\ln \left(f(k)-k f^{\prime}(k)\right)}{f^{\prime}(k)}=0 .^{31}$

Lemma 16. If $f(0)=0$, ass. 5 is equivalent to each of the 2 following properties:

\footnotetext{
${ }^{31}$ This includes the classical Cobb-Douglas case.
} 
(a) $\forall \theta>0,\left[f(k)-k f^{\prime}(k)\right] e^{\theta f^{\prime}(k)} \underset{k \rightarrow 0}{\longrightarrow} \infty$;

(b) $\forall \theta>0, \liminf _{k \rightarrow 0}\left[f(k)-k f^{\prime}(k)\right] e^{\theta f^{\prime}(k)}>0$;

Under ass. 5 , if $h_{k}$ converges exponentially in $|\mathfrak{r}|$ to $\infty$ at rate almost $\theta>0$ when $k \rightarrow 0$, so does $\left[f(k)-k f^{\prime}(k)\right] h_{k}$.

Proof. $\mathrm{b} \Rightarrow$ ass. 5: Passing to logarithms, we have $\forall \theta>0, \exists k_{\theta}, \exists M: \forall k \leq k_{\theta}, \theta f_{k}^{\prime}+$ $\ln \left(f_{k}-k f_{k}^{\prime}\right) \geq M$. Since $f(0)=0, \ln \left(f_{k}-k f_{k}^{\prime}\right) \rightarrow-\infty$, so $\frac{\theta f_{k}^{\prime}}{\ln \left(f_{k}-k f_{k}^{\prime}\right)}-1 \geq-\frac{1}{2}$ if $k \leq k_{\theta}$ and $\left|\ln \left(f_{k}-k f_{k}^{\prime}\right)\right| \geq 2 M$. So, $\lim _{i n f} \sin _{k \rightarrow 0} \frac{f_{k}^{\prime}}{\left|\ln \left(f_{k}-k f_{k}^{\prime}\right)\right|} \geq \frac{1}{2 \theta}, \forall \theta>0$, thus ass. 5 . ass. $5 \Rightarrow$ a: Since $f(0)=0, \ln \left(f_{k}-k f_{k}^{\prime}\right) \rightarrow-\infty$, so $<0$ for $k$ sufficiently small. Thus, since $f^{\prime}(k)>0$ for $k$ sufficiently small, ass. 5 implies $\forall M \exists k_{0}: \forall k \leq k_{0}, \mid \ln \left(f_{k}-\right.$ $\left.k f_{k}^{\prime}\right) \mid \geq M$ and $f_{k}^{\prime} \geq M\left|\ln \left(f_{k}-k f_{k}^{\prime}\right)\right|$. So $\ln \left(\left[f(k)-k f^{\prime}(k)\right] e^{\theta f^{\prime}(k)}\right)=\theta f^{\prime}(k)-$ $\left|\ln \left[f(k)-k f^{\prime}(k)\right]\right| \geq(M \theta-1)\left|\ln \left[f(k)-k f^{\prime}(k)\right]\right|$, which goes to $\infty$ when $M \geq \frac{2}{\theta}$.

$\mathrm{a} \Rightarrow \mathrm{b}$ trivially.

Ass. 5 implies $f^{\prime}(0)=\infty$ if $f(0)=0$. Now $\mathfrak{r}=R-f_{k}^{\prime}$, so when $k \rightarrow 0,|\mathfrak{r}| \rightarrow \infty$, and $e^{\theta f_{k}^{\prime}}$ in a equals $e^{\theta|\mathfrak{r}|}$ up to a constant factor. $\forall \varepsilon>0$ and for $k$ sufficiently large, by assumption $h_{k} \geq e^{(\theta-\varepsilon)|\mathfrak{r}|}$ and, by a, $\left(f_{k}-k f_{k}^{\prime}\right) e^{\varepsilon|\mathfrak{r}|}>1$, so $\left(f_{k}-k f_{k}^{\prime}\right) h_{k} \geq$ $e^{(\theta-2 \varepsilon)|\mathfrak{r}|}$. And since $f_{k}-k f_{k}^{\prime} \rightarrow 0$, clearly $\left(f_{k}-k f_{k}^{\prime}\right) h_{k} \leq e^{\theta|\mathfrak{r}|}$ for $k$ large.

Corollary 17. Assume $a_{\varphi}<\sigma, f(0)=0$, and ass. 5. Then

$$
\lim _{k \rightarrow 0}\left[f(k)-k f^{\prime}(k)\right] \tilde{H}_{\varphi}(\mathfrak{r})=-\infty
$$

The convergence is exponential in $\mathfrak{r}$ at rate almost $\min \{1, \sigma\}-a_{\varphi}$.

Proof. Note that ass. 5 and $f(0)=0$ implies $f^{\prime}(0)=\infty$. Use then lemma 15.b and the last statement of lemma 16.

Theorem 3. Assume $f$ is analytic on $\mathbb{R}_{++}, f(0)=0$ and ass. 5 is satisfied. The number of BGE of the basic model, as well as that of the general model with $\omega=0$, is finite, except possibly in cases a and c below when there exists no appropriate $\varepsilon$. More precisely, let $N$ be the number of positive roots (counting multiplicities) of the equilibrium equation $G(k) \stackrel{\text { def }}{=} \tilde{H}_{\Xi}(\mathfrak{r})-k \mathfrak{r}$. Then

(a) If $\sigma \leq \min \left\{a_{\varphi}, a_{E}\right\}, N$ is even if $\Omega<0$ and odd if $\Omega>0$, or if $\Omega=0$ and either $\sigma \neq a_{E}$ or $E_{s} \leq 0$ on $[\sigma, \sigma+\varepsilon]$.

(b) If $a_{\varphi}<\min \left\{a_{E}, \sigma\right\}, N$ is even.

(c) If $a_{E} \leq a_{\varphi}$ and $a_{E}<\sigma$ then $N$ is even (resp., odd) if $\exists \varepsilon>0$ s.t. $E_{s} \geq 0$ (resp., $E_{s} \leq-\varepsilon \varphi_{s}$ ) a.e. on $\left[a_{E}, a_{E}+\varepsilon\right]$.

Comment 31 . The case $E_{s} \leq-\varepsilon \varphi_{s}$, which plays a rôle only if $a_{E}=a_{\varphi}$ (else it boils down to $E_{s} \leq 0$ ), may seem non-generic. But models where taxing starts when working does, with tax at least an $\varepsilon$-fraction of income, are not that implausible.

Proof. Since the assumptions imply $f^{\prime}(0)=\infty$, BGE correspond, by rem. 23 and cor. 13 , to the positive solutions of $G(k)=\tilde{H}_{E}(\mathfrak{r})+\left(f_{k}-k f_{k}^{\prime}\right) \tilde{H}_{\varphi}(\mathfrak{r})-k \mathfrak{r}=0$ where also $\mathcal{N} \geq 0$. Since $f$ is (real-)analytic on $\mathbb{R}_{++}$, and so are $\tilde{H}_{E}$ and $\tilde{H}_{\varphi}$ by lemma 27 , thus $G(k)$ is real-analytic on $\mathbb{R}_{++}$as well.

As $k \rightarrow \infty, \mathfrak{r}=R-f_{k}^{\prime} \rightarrow R-f_{\infty}^{\prime}>0$ by ass. 4 . Since $\tilde{H}_{h}\left(R-f_{\infty}^{\prime}\right)$ is finite and since $\lim _{k \rightarrow \infty} \frac{f_{k}}{k}-f_{k}^{\prime}=f_{\infty}^{\prime}-f_{\infty}^{\prime}=0, \lim _{k \rightarrow \infty} \frac{G(k)}{k}=f_{\infty}^{\prime}-R<0$, and $G$ is not identically zero. Thus its set of positive roots is discrete, and bounded, and the multiplicity of each root is well-defined and finite.

So to prove finiteness, suffices to show that 0 is not a limit point of a sequence of roots. We will thus evaluate the sign of $G$ as $k$ approaches zero. If the sign is negative (resp., positive), the number of roots counting multiplicities is even (resp., odd).

Note that $\mathfrak{r} \underset{k \rightarrow 0}{\longrightarrow}-\infty$ since $f_{0}^{\prime}=\infty$, and $k \mathfrak{r}=k R-k f_{k}^{\prime} \underset{k \rightarrow 0}{\longrightarrow} 0$ since $k f_{k}^{\prime} \underset{k \rightarrow 0}{\longrightarrow} 0$. 
a: If $\sigma \leq \min \left\{a_{E}, a_{\varphi}\right\}$ then by lemma 15.a, $\tilde{H}_{E}$ converges to $\Omega$ while the other two terms $\left[\left(f_{k}-k f_{k}^{\prime}\right) \tilde{H}_{\varphi}\right.$ and $\left.-k \mathfrak{r}\right]$ converge to zero. Hence if $\Omega \neq 0$ the sign of $G$ is that of $\Omega$. If $\Omega=0$, assume first $\sigma<a_{E}$. Then the first term in $G, \tilde{H}_{E}$, converges exponentially (in $\mathfrak{r}$ ) to 0 by lemma 15 .a, and $\tilde{H}_{\varphi}$ converges to 1 , so the sum of the last 2 terms, which are positive, is of the order of $f_{k}-k f_{k}^{\prime}-k\left(R-f_{k}^{\prime}\right)=f_{k}-R k$, i.e., of the order of $f_{k}$ since $f_{0}^{\prime}=\infty$. Suffices thus to show that $f_{k}$ converges slower than exponentially (in $\mathfrak{r}$ ) to 0, i.e., that $f_{k} e^{\varepsilon f_{k}^{\prime}} \rightarrow \infty \forall \varepsilon>0$, which is by lemma 16.a. Finally, if $\sigma=a_{E} \leq a_{\varphi}$, the first term is $-\digamma(\mathfrak{r}) \int_{\sigma}^{1} e^{\mathfrak{r} s} E_{s} d s$, it converges exponentially to zero by lemma 15 .a, and because $\mathfrak{r}<0$, when $E \leq 0$ on $[\sigma, \sigma+\varepsilon]$, it yields a positive term on that interval. Thus, since the other 2 terms are positive, the argument is the same as in the previous case.

b: If $a_{\varphi}<\sigma \leq a_{E}$, then $\tilde{H}_{E}(\mathfrak{r}) \underset{\mathfrak{r} \rightarrow-\infty}{\longrightarrow} \Omega$ by lemma 15.a, $\left(f_{k}-k f_{k}^{\prime}\right) \tilde{H}_{\varphi}(\mathfrak{r})$ converges to $-\infty$ (by cor. 17), and $k \mathfrak{r} \rightarrow 0$ (cf. supra), so $G$ is negative.

If $a_{\varphi}<a_{E}<\sigma$ then by cor. $17\left(f_{k}-k f_{k}^{\prime}\right) \tilde{H}_{\varphi}(\mathfrak{r})$ converges to $-\infty$ exponentially at rate almost $\min \{\sigma, 1\}-a_{\varphi}$, which is higher than the rate $\left(\min \{\sigma, 1\}-a_{E}\right)$ of convergence of $\tilde{H}_{E}($ to $\pm \infty)$ by lemma 15 .b, hence $G$ is negative (since $\left.-k \mathfrak{r} \leq \varepsilon|\mathfrak{r}|\right)$.

c: Let $b=a_{E}+\varepsilon$. If $a_{E}<\sigma \leq a_{\varphi}$, then by lemma 15.a, $\left(f_{k}-k f_{k}^{\prime}\right) \tilde{H}_{\varphi}(\mathfrak{r})$ converges to zero with $f_{k}-k f_{k}^{\prime}$, and by lemma 15.b $\tilde{H}_{E}$ converges to $\pm \infty$ depending on the sign of $E$ on $\left[a_{E}, b\right]$, so the sign of $G$ is the opposite of that one.

If $a_{E} \leq a_{\varphi}<\sigma$ and $E$ is positive on $\left[a_{E}, b\right]$, then $\tilde{H}_{E}$ and $\tilde{H}_{\varphi}$ converge to $-\infty$ and $G$ is negative. If $a_{E}<a_{\varphi}<\sigma$ and $E$ is negative on $\left[a_{E}, b\right]$, then $\tilde{H}_{E}$ converges faster (at rate almost $\min \{\sigma, 1\}-a_{E}$ ) than $\left(f_{k}-k f_{k}^{\prime}\right) \tilde{H}_{\varphi}(\mathfrak{r})$, by cor. 17 , hence $G$ is positive. So we get the same conclusion as in the previous paragraph.

And if $a_{E}=a_{\varphi}<\sigma$ with $E_{s} \leq-\varepsilon \varphi_{s}$, then for any $k>0$ s.t. $f_{k}-k f_{k}^{\prime}<\varepsilon / 2$, $\Xi_{s}=E_{s}+\left(f_{k}-k f_{k}^{\prime}\right) \varphi_{s} \leq-\frac{\varepsilon}{2} \varphi_{s}$ on $\left[a_{E}, b\right]$. So $G$ has the sign of $\varphi$ on $\left[a_{\varphi}, b\right],>0$.

Corollary 18. Assume $f$ is analytic on $\mathbb{R}_{++}, f(0)=0$ and ass. 5 is satisfied. If, in addition, $E \geq 0$, then the number of BGE, counting multiplicities, is odd iff $\sigma \leq \min \left\{a_{\varphi}, a_{E}\right\}$, else even.

Proof. By thm. 3, since for $E \geq 0, \mathcal{N} \geq 0$, so $N$ is the number of BGE.

Comment 32. By contrast, in the discrete time exchange models of the literature $[12,14]$, with time truncated at zero, the number is claimed to be always even.

Similarly [2, prop. 3 and thm. 2] imply that there always exists a pure BGE in case of Cobb-Douglas production technology and no transfers, thus contradicting our graph 3 exhibiting an economy that has only a GRE for all values of $\alpha$. The graph, in turn, illustrates cor. 18.

Cor. 18 complements the claim in [13] about existence and uniqueness of pure BGE when $\sigma \geq 1\left(\geq \min \left\{a_{\varphi}, a_{E}\right\}\right)$ and when there are no transfers.

Comment 33. If, with stationary endowments, there are non-BGE equilibria, then they form a continuum, by shift invariance.

\section{Conclusions}

We think of this model as a template for how to do policy analysis in a mostly analytical way. Policy here is expressed in terms of transfers (endowments), i.e., of their distribution across age-groups at each instant of time. We rely only on the most standard definition of general equilibrium, i.e., optimisation by agents, without any ad-hoc assumption, like forcing equilibrium variables to belong to specific spaces, or to be non-zero. Our equilibrium characterisation suffices for the proof in [18] of the validity of comparative statics (i.e., no indeterminacy) in this model. 
Not all classical results extend, for example, the parity of BGE depends on the relative magnitude of the minimal working and tax ages, and the intertemporal elasticity of substitution.

Several open questions still remain. First, do there (generically) exist equilibria with stationary transfers that are not BGE? Do they all converge to some BGE at $+\infty$ and $-\infty$ ? If so, what is the directed graph induced among BGE? Next, what is a complete characterisation of equilibria of the general model, where some irreversibility constraints might be binding? Tackling this question is a prerequisite for handling the several-commodity model of [20]. Finally, is there a generalisation of Gale's dichotomy to all equilibria (with non-stationary endowments), e.g., is Pareto implied by non-zero net assets? The last question is partially answered in [17]: the GRE is Pareto in this model.

\section{Appendix A. Aggregation of demand}

To aggregate consumption the classic integration of correspondences $[1,7]$ does not apply, as consumption bundles are equivalence classes of arbitrary $\overline{\mathbb{R}}_{+}$-valued Lebesgue-measurable functions, so do not lie in any vector space. We use the following very close analog.

Notation A.1. Let $\mathcal{M}$ (or $\mathcal{M}^{\mathbb{R}}$ to denote the domain) be the set of all equivalence classes of arbitrary $\overline{\mathbb{R}}_{+}$-valued Lebesgue-measurable functions with the topology of convergence in measure on all compact sets, for any fixed distance on $\overline{\mathbb{R}}_{+}$.

The topology is independent of the distance, and is Polish, so the usual measurable selection theorems hold.

Definition 6. Given a measurable space $(X, \mathcal{X}, \mu)$, the integral of a measurable $\mathcal{M}$-valued function $x \mapsto F_{x}$ is the unique point $G$ in $\mathcal{M}$ s.t. $\forall p \in \mathcal{M}, \int p(t) G(t) d t=\iint p(t) F_{x}(t) d t \mu(d x)$, with the usual measure-theoretic convention that $0 \times \infty=\infty \times 0=0$.

The integral of a measurable $\mathcal{M}$-valued correspondence with a.e. well-defined and nonempty values is the set of integrals of all its measurable (a.e.)-selections.

To prove the above is well-defined (and to show how it is used), observe that by Doob's [1953] classical martingale argument, there exists for any such $F$ a jointly measurable function $f(x, t)$ s.t. $f(x, \cdot) \in F_{x} \forall x$ (use first a homeomorphism of $\overline{\mathbb{R}}_{+}$with $[0,1]$ to reduce to the case where $\left.\sup _{x}\left\|F_{x}\right\|_{\infty} \leq 1\right)$. Fubini's theorem implies then that $\int f(x, t) \mu(d x)$ satisfies the requirements for $G$. Uniqueness is obvious.

Conversely, given any jointly measurable $\overline{\mathbb{R}}$-valued function $f(x, t), F: x \mapsto f(x, \cdot)$ is a measurable $\mathcal{M}$-valued map. Indeed, assume first $f$ is bounded; then $F$ is measurable to $L_{\infty}$ with the weak*-topology, since bounded subsets there are compact metric. But those bounded subsets are Polish for the topology of convergence in measure on compact sets, so the Borel structure is the same. For general $f$, approximate it by the sequence $f_{n}=\max \{-n, \min \{f, n\}\}, n \in \mathbb{N}$.

Note finally that, $G$ being well-defined, it suffices to check the equality in the definition with indicator functions of compact sets for $p$.

\section{Appendix B. The evaluation of profits}

Profits are an economic concept, but not a mathematical concept. Its translation to a formal concept for the profits of investment firms that can make capital transactions in continuous time is by no means obvious. The purpose of this appendix is to give a formal definition of the profits of investment firms, and to motivate it by the economic concept. Assumption 2, the description of investment firms, is a standing assumption here.

B.1. The "hot potato" example. To illustrate the need of assumption 3.d for the correct evaluation of profits, consider the following example: $(F, \mathcal{F}, \mu)$ equals $[-1,1]$ with Lebesgue measure; take as endowments $\omega_{x, s}$ the negative of what would be the real wages (i.e., in commodity terms) under the GRE with 0 endowments. The "proposed equilibrium" is the GRE, except that $p_{t}$ is doubled for $t<0$. Let $t_{n}=\frac{-1}{n+1}\left(\right.$ and $\left.t_{0}=-\infty\right)$; for $t_{n-1} \leq$ 
$t<t_{n}$, all capital is held and investment is done by the firms $f$ with $t_{n-1} \leq f<t_{n}$ (say uniformly spread), and for $t \geq 0$, by the firms with $f \geq 0$. Then all firms make 0 profits, while on the aggregate they make a big loss (at time 0 ). Further, the technological constraint $K_{t}^{f} \geq 0$ prevents a profitable deviation by any firm. (Using the capital accumulation equation to depreciate negative holdings of plant and equipment would seem far-fetched.)

The same example could be re-cast with finitely many firms: take 2 firms active before time 0 , exchanging the capital between them at times $t_{n}$, and a third, active from time 0 on.

Thus we need a reliable way to evaluate profits, that aggregates properly: the equilibrium criterion for investment firms is that every coalition of them makes 0 profits on any bounded interval of time (in particular, every coalition where ess sup $\max \left\{\left|t_{0}^{f}\right|,\left|t_{1}^{f}\right|\right\}<\infty$ makes zero profits), and none can make more with production plans having well-defined profits; so profits of coalitions are fundamental, and must be completely unambiguous. To "aggregate well" is in particular to ensure this criterion is equivalent to asking the same for a.e. firm; thus, for any coalition (in $\mathcal{F}$ ) profits on such an interval from its aggregate activity must equal the integral of its members' profits on this interval. Further, there are at least 2 ways to evaluate profits (sect. B.4 and B.6), applicable to different classes of functions.

Definition 7. A formula $\pi(K, I)$ for profits aggregates well over a plan $\left(K^{f}, I^{f}\right)$ if $\forall S \in \mathcal{F}$, $\pi\left(\int_{S} K^{f} \mu(d f), \int_{S} I^{f} \mu(d f)\right)=\int_{S} \pi\left(K^{f}, I^{f}\right) \mu(d f)$.

Notation. $M(X)$ is the space of bounded measures on a measurable space $X$. $\mathcal{B}(X)$ is the Borel $\sigma$-field on a topological space $X . \epsilon_{x}$ is the unit mass at point $x$.

B.2. The variation. Let $V_{a, b}(g)=\sup _{n} \sup _{a \leq t_{i-1} \leq t_{i} \leq b} \sum_{i=1}^{n}\left\|g\left(t_{i}\right)-g\left(t_{i-1}\right)\right\|$, the variation of $g$ on $[a, b] . g$ has locally bounded variation if $\forall[a, b], V_{a, b}(g)<\infty . V=V_{-\infty, \infty} . X$ has locally integrable variation if $\forall[a, b]$, the upper integral $\int^{*} V_{a, b}(X(f, \cdot)) \mu(d f)<\infty$.

Lemma 17. If $X(f, t)$ is jointly measurable on $\mathcal{F} \otimes \mathcal{B}(\mathbb{R})$, then $V_{a, b}(X(f, \cdot))$ is measurable.

Proof. Suffices to prove $V^{n}(f) \stackrel{\text { def }}{=} \sup _{a \leq t_{i-1} \leq t_{i} \leq b} \sum_{i=1}^{n}\left|X\left(f, t_{i}\right)-X\left(f, t_{i-1}\right)\right|$ is measurable. Let $T=\left\{\left(t_{i}\right)_{i=0}^{n} \mid a \leq t_{i-1} \leq t_{i} \leq b\right\}$, and, for $\tau \in T, Y_{f, \tau}=\sum_{i=1}^{n} \mid X\left(f, t_{i}\right)-$ $X\left(f, t_{i-1}\right) \mid . Y$ is $\mathcal{F} \otimes \mathcal{B}(T)$-measurable, so $\left\{f \mid V^{n}(f)>\alpha\right\}$ is the projection of $\{(f, \tau)$ $\left.Y_{f, \tau}>\alpha\right\} \in \mathcal{F} \otimes \mathcal{B}(T)$, hence measurable since $\mathcal{F}$ is complete and $T$ compact metric.

B.3. Transactions and production sets. The most standard concept of profits is that of the value of net output under the price system. We need thus the cumulative net volume of capital sales $H_{t}$ for a production plan $\left(K_{t}, I_{t}\right)$. It is indeed $d H_{t}$ that represents the corresponding net output vector of (dated) capital goods. Assume $K_{t}$ has bounded variation. If capital is purchased only once at time $t_{0}<t$ then the capital accumilation equation (cor. 2) becomes $e^{\delta t} K_{t}-\int_{-\infty}^{t} e^{\delta s} I_{s} d s=-d H_{t_{0}} e^{\delta t_{0}}$ (the sign since $H$ represents sales). To account for purchases at all times $t_{0}<t$ we integrate w.r.t. $-d H_{t_{0}}$, which yields $K_{t}=$ $e^{-\delta t} \int_{-\infty}^{t} e^{\delta s}\left[I_{s} d s-d H_{s}\right]$, so $d H_{t}=I_{t} d t-e^{-\delta t} d\left(e^{\delta t} K_{t}\right)$ and $H_{t}=\int_{-\infty}^{t}\left(I_{s}-\delta K_{s}\right) d s-K_{t} \cdot{ }^{32}$ So:

Definition 8. $H_{t} \stackrel{\text { def }}{=} \int_{0}^{t}\left(I_{s}-\delta K_{s}\right) d s-K_{t}$ denotes cumulative net sales of capital.

Lemma 18. For $I_{t} \in L_{1}^{\text {loc }}$ (resp., $I_{t}^{f}$ as in ass. 3.a), $H_{t}$ (resp., $H_{t}^{f}$ ) has locally bounded (resp., locally integrable) variation iff $K_{t}$ (resp., $K_{t}^{f}$ ) does.

Proof. By rem. $4, I_{s}^{f}-\delta K_{s}^{f}$ is a.e. in $L_{1}^{\text {loc }}$, and by ass. 3.a, it is locally jointly integrable, so its primitive has resp. locally bounded and locally integrable variation.

Comment 34. For a feasible plan, let $K=\int K^{f} \mu(d f)$, and similarly for $I$ and $H$. Then $H$ must be constant, since no other sector buys or sells capital. The equation in def. 8 yields then the capital accumulation equation.

\footnotetext{
${ }^{32}$ Alternatively, by rem. $4, I_{t}-\delta K_{t}$ is in $L_{1}^{\text {loc }}$, and, if there are no intervening sales, $K_{t}$ is locally absolutely continuous and a.e. differentiable with $I_{t}-\delta K_{t}$ as derivative, by cor. 2, so that $K_{b}-K_{a}=\int_{a}^{b}\left(I_{t}-\delta K_{t}\right) d t: I_{t}-\delta K_{t}$ is net investment. Therefore, $K_{t}+H_{t}$, which is the total amount of capital accumulated by the firm up to time $t$, including capital already sold and excluding capital bought, must equal its total past net investment, $\int^{t}\left(I_{s}-\delta K_{s}\right) d s$.
} 
The production set $Z_{f}$ of an investment firm $f$ is its set of feasible outputs of dated goods: capital stock sold $d H_{t}$, capital services sold $K_{t}$, and investment bought $I_{t}{ }^{33}$

Since traditionally the aggregate production set $Z$ is defined as the integral of the correspondence $f \mapsto Z_{f}$, we still need to specify a space embedding the $Z_{f}$. This needs some care, to ensure that $Z$ satisfies ass. $1 .^{34}$

Definition 9. (a) For $N \in \mathbb{N}$, let $p_{N}(I) \stackrel{\text { def }}{=}\left\|I \mathbb{1}_{[-N, N]}\right\|_{1}, p_{N}(K) \stackrel{\text { def }}{=} V\left(K \mathbb{1}_{[-N, N]}\right)$. $P$ is the locally convex space $\left\{(H, K,-I) \mid H_{t}=\int_{0}^{t}\left(I_{s}-\delta K_{s}\right) d s-K_{t}, \forall N, p_{N}(I)<\right.$ $\infty, p_{N}(K)<\infty, k_{t}$ bounded $\}$, with the $p_{N}(I), p_{N}(K)$, and $\|k\|_{L_{1}(\nu)}(\nu \in M(\mathbb{R}))$ as family of semi-norms, indexed by $N$ and $\nu$, and with $k_{t}$ as defined in sect.2.5.2.

(b) For $f \in F$, the production set $Z_{f} \stackrel{\text { def }}{=}\left\{(H, K,-I) \in P \mid K_{t} \geq 0, K_{t}=I_{t}=\right.$ 0 for $\left.t \notin\left[t_{0}^{f}, t_{1}^{f}\right]\right\}$. The aggregate production set $Z \stackrel{\text { def }}{=} \int Z_{f} \mu(d f)$, with $P$-valued integrals understood as Bochner integrals.

Remark 35. Endowing $H$ with the same topology as $K$, one sees that $H$ is a continuous linear function of $(I, K)$. Further, $K_{t}=-H_{t}+e^{-\delta t} \int_{0}^{t} e^{\delta s}\left(I_{s}-\delta H_{s}\right) d s$ shows that also $K$ is a continuous linear function of $(I, H)$. Finally, $H+K$ lies in the closed subspace of absolutely continuous finctions; on this closed subspace, $I$ is a continuous linear function of $(H, K)$.

Remark 36. Recall, a function $f$ with values in a locally convex space $E$ is Bochner integrable iff $f$ takes values a.e. in some separable subspace of $E, \varphi \circ f$ is measurable $\forall \varphi$ in the dual $E^{\prime}$, and the upper integral $\int^{*}(p \circ f)<\infty$ for each $p$ in a sub-basis of semi-norms for $E$. The same holds then for any continuous semi-norm on $E$, and $\int f$ is defined in the algebraic dual of $E^{\prime}$ by $\varphi \circ \int f=\int(\varphi \circ f) \forall \varphi \in E^{\prime}$. If $E$ is complete, $\int f \in E .^{35}$

Thus, to justify the above definition, we must prove $P$ is complete.

Lemma 19. $P$ is a complete locally convex space.

Proof. $P$ being essentially the product of its $I$ and $K$ factors, separate proofs suffice.

For $I$, this is the space $L_{1}^{\text {loc }}$, and results immediately from $L_{1}$ being a Banach space.

For $K$, since $p_{N}$ is stronger than the $\sup _{[-N, N]}$ norm, a Cauchy net $k_{\alpha}$ converges locally uniformly, hence pointwise, say to $k$. Then $p_{N}\left(k_{\alpha}-k\right) \rightarrow 0$ follows from the implication $y_{\alpha} \rightarrow y$ pointwise $\Rightarrow V(y) \leq \liminf V\left(y_{\alpha}\right)$.

We now prove that $k$ is bounded. Else, up to a sign change, $\forall n \exists t_{n}: k\left(t_{n}\right) \geq 2^{n}$. Let then $\nu_{n}=\sum_{i<n} 2^{-n} \epsilon_{t_{n}}$ : since $\nu_{n}$ has bounded support, $K \mapsto \int k d \nu_{n}$ is a linear functional $\phi_{\nu_{n}}$ on $P$ dominated by some $p_{N}$, thus, by the above, $\phi_{\nu_{n}}\left(k_{\alpha}\right) \rightarrow \phi_{\nu_{n}}(k)$. But $\nu_{n} \leq \nu_{\infty}$ implies that, since $k_{\alpha}$ is Cauchy in $\|\cdot\|_{L_{1}\left(\nu_{\infty}\right)}$, the $k_{\alpha}$ are bounded and converge uniformly on the $\nu_{n}$ : their pointwise limit $\phi_{\nu_{n}}(k)$ is also bounded on the $\nu_{n}$, contradicting $\phi_{\nu_{n}}(k) \geq \sum_{i<n} 2^{-n} 2^{n}=n$. So $k$ is bounded. Hence, having locally bounded variation, it corresponds to a point in $P$. Remains to show that $\left\|k_{\alpha}-k\right\|_{L_{1}(\nu)} \rightarrow 0 \forall \nu \in M(\mathbb{R})$.

Repeating the previous argument with $\nu_{N}$ the restriction of $\nu$ to $[-N, N]$ shows that $\phi_{\nu_{N}}\left(k_{\alpha}\right) \rightarrow \phi_{\nu_{N}}(k)$ uniformly in $N$. And since the $k_{\alpha}$ and $k$ are bounded, $\lim _{N} \phi_{\nu_{N}}\left(k_{\alpha}\right)$

\footnotetext{
${ }^{33}$ In principle one could equivalently forbid capital transactions; firms would then at the beginning of their life-time build up capital stock by buying investment, and at the end deplete it by disinvesting and selling the investment good. But in the absence of a market for capital there can be no price for capital, so all prices would be prices of flows, and hence only equivalence classes of measurable functions, making the whole language a bit more cumbersome and less transparent. Since further this trick of transferring capital to another firm by disinvesting it and selling the investment good is quite artificial and unappealing, we chose the present formulation.

${ }^{34}$ Indeed, the initial condition is a technological restriction ("how much coal was initially in the ground"), so it has to be reflected in the production sets. And it is naturally in $Z$ that it has to appear (and not as a time-dependent restriction on how much capital can be used in instantaneous production). Since obviously it has no counterpart in the $Z_{f}$, which have compact support, it must be implied by the aggreation procedure., i.e., by the concept of integral-and thus ultimately by the choice of the embedding space and its topology. Thus, using a classical approach of integrating firms' production sets makes the treatment a bit more technical (adding the boundedness requirement on the $k_{t}$, and the semi-norms $\|k\|_{L_{1}(\nu)}$, and thus leaving the realm of Fréchet spaces), and hides behind abstract definitions of spaces what is now a very clear and explicit economic assumption, the initial condition.

${ }^{35}$ The proof of $[11$, thm. 8.14.14 p. 570] holds also in abstract measure theory.
} 
$=\int k_{\alpha}(t) \nu(d t) \stackrel{\text { def }}{=} \phi_{\nu}\left(k_{\alpha}\right)$, and the same for $k$. Thus $\phi_{\nu}\left(k_{\alpha}\right) \rightarrow \phi_{\nu}(k) \forall \nu \in M(\mathbb{R})$. In particular, $\int k_{\alpha}(t) f(t) \nu(d t) \rightarrow \int k(t) f(t) \nu(d t) \forall f \in L_{\infty}(\nu)$. But the $k_{\alpha}$ are uniformly Cauchy on the unit ball of $L_{\infty}(\nu)$, so the convergence is uniform: $k_{\alpha} \rightarrow k$ in $L_{1}(\nu)$.

Lemma 20. A subspace of $P$ is contained in a separable subspace iff the $K$ 's are all dominated by a single measure - i.e., all discontinuities fall into a single countable set, and the measures represented by the continuous parts belong to some fixed $L_{1}^{\text {loc }}(\nu)$ space.

Proof. Separability of $L_{1}^{\text {loc }}$ results from that of $L_{1}$, hence $I$ components are always separable. For $K$, given a dense sequence one constructs immediately the countable set of discontinuities, and then, from the continuous parts, (locally) the dominating measure $\nu$. Conversely, any such subspace is separable - again the separability of $L_{1}^{\text {loc }}(\nu)$ for the continuous parts, and basically of $l_{1}$ for the rest.

Lemma 21. $f \mapsto K_{t}^{f} \geq 0$ is Bochner-integrable iff it is measurable for each fixed $t$, separably valued (cf. lemma 20), has locally integrable variation, and $t \mapsto \int k_{t}^{f} \mu(d f)$ and each $k_{t}^{f}$ are bounded. $K_{t}^{f}$ is then $\mathcal{F} \otimes \mathcal{B}(\mathbb{R})$-measurable, so $p_{N}\left(K^{f}\right)$ and $\left\|k^{f}\right\|_{L_{1}(\nu)}$ are measurable.

Proof. Necessity: evaluation at $t$ is a continuous linear functional, separability is by lemma 20, boundedness is because $k$ 's in $P$ are bounded, and by lemma 19 , implying that $\int K_{t}^{f} \mu(d f) \in P$, and the variation is locally integrable by definition.

$\mathcal{F} \otimes \mathcal{B}(\mathbb{R})$-measurability: Since $K_{t}^{f}$ has locally bounded variation, it can be decomposed into its right-continuous regularisation, which is $\mathcal{F} \otimes \mathcal{B}(\mathbb{R})$-measurable, and the difference, which is non-null only at countably many $t_{n}$ (lemma 20 ), and clearly $\mathcal{F}$-measurable at each $t_{n}$; hence the $\mathcal{F} \otimes \mathcal{B}(\mathbb{R})$-measurability of $K_{t}^{f}$, so $p_{N}\left(K^{f}\right)$ is measurable by lemma 17 .

Sufficiency: For the measurability of any continuous linear functional $\phi$, let $\phi_{n}(k)=$ $\phi\left(k \mathbb{1}_{[-n, n]}\right)$ : since $\phi_{n} \rightarrow \phi$ pointwise, suffices to prove measurability of $\phi_{n}$. Note that, on our separable subspace, $\phi_{n}(h)$ has the form $\int h d \nu+\int_{\mathbb{T}_{\delta}} g d h$ (cf. fn. 37), for some $\nu \in M(\mathbb{R})$ and a bounded Borel function $g$ with compact support on $\mathbb{T}_{\delta}$, because by separability, all measures $d h$ fall into some $L_{1}$ space on $\mathbb{T}_{\delta} \cap[-n, n]$, so continuous linear functionals are represented by a bounded Borel function $g$. The measurability follows then, by first approximating $g$ as an a.e. limit of a uniformly bounded sequence of continuous functions with compact support on $\mathbb{T}_{\delta}$, then noting that, $\mathbb{T}_{\delta}$ being totally disconnected, such a continuous function is a limit of continuous simple functions: this reduces the problem to the case where $g$ is the indicator of a clopen set in $\mathbb{T}_{\delta}$, i.e., a finite union of compact open intervals $] a_{2 i}, a_{2 i+1}\left[\right.$ in $\mathbb{T}_{\delta}\left(a_{i}<a_{i+1} \in \mathbb{R}\right)$; for such an interval $] a, b[$, the integral of $g d h$ is simply $h(b)-h(a)$. Hence the measurability. Integrability of the $p_{N}$ follows from its measurability and the locally integrable variation. As to that of the $\left\|k^{f}\right\|_{L_{1}(\nu)}$, $\left\|k^{f}\right\|_{L_{1}(\nu)}=\int k_{t}^{f}|\nu|(d t)$, so $\int\left\|k^{f}\right\|_{L_{1}(\nu)} \mu(d f)=\iint k_{t}^{f} \mu(d f)|\nu|(d t)$ by Fubini's theorem (joint measurability of $\left.K_{t}^{f}\right), \leq\|\nu\| \sup _{t} k_{t}<\infty$ by the boundedness of the integral $k$.

The Bochner integral - the most restrictive - was chosen because it is the most classical, and in order to give as safe aggregation as possible. We first prove that it is indeed sufficient: that the implied allocations - the integrable selections from $Z_{f}$ - do satisfy ass. 3 and that $K_{t}^{f}$ has locally integrable variation. And next, that this restrictive approach entails no hidden restriction on aggregates: $Z=\{(H, K,-I) \in P \mid K \geq 0\}$.

Proposition 2. For Bochner-integrable $\left(H_{t}^{f}, K_{t}^{f},-I_{t}^{f}\right) \in Z_{f}, I_{t}^{f}$ has a $\mathcal{F} \otimes \mathcal{B}(\mathbb{R})$-measurable version (choosing 1 point in the equivalence class $\forall f$ ) s.t. $\int I_{t}^{f} \mu(d f)$ exists for all $t$. For any such version (even only $d \mu \times d \lambda$-measurable), $\int I_{t}^{f} \mu(d f)$ is a version of the integral, ass. 3 holds, $K_{t}^{f}$ is $\mathcal{F} \otimes \mathcal{B}(\mathbb{R})$-measurable and has locally integrable variation, and, with $K \stackrel{\text { def }}{=} \int K^{f} \mu(d f), K_{t_{-}}=\int K_{t_{-}}^{f} \mu(d f) \forall t$, and the same at $t_{+}$and $t$, and ass. 1 holds.

Proof. Denote the integral by $(H, K,-I)$. By Doob's [1953] classical martingale argument, $I_{t}^{f}$ has a jointly measurable version. Modify this s.t. $I_{t}^{f}=0 \forall t \notin\left[t_{0}^{f}, t_{1}^{f}\right]$. $I_{t}^{f}$ is then locally in $t$ jointly integrable, since the integral of a norm is finite for a Bochner integral. Thus for a.e. $t I_{t}^{f}$ is integrable w.r.t. $f$. Let $I_{t}^{f}=0$ identically for the other $t$ : now $I_{t}=\int I_{t}^{f} \mu(d f)$ exists for all $t$, and is, by the local joint integrability, a version of the integral.

$K_{t}^{f}$ is $\mathcal{F} \otimes \mathcal{B}(\mathbb{R})$-measurable and has locally integrable variation by lemma 21 . A fortiori it is also locally in $t$ jointly integrable, since it is jointly measurable and its sup-norm on 
$[-N, N]$ is integrable. Finally $K_{t}=\int K_{t}^{f} \mu(d f) \forall t$, since evaluation at $t$ is a continuous linear functional. And since $K_{t}^{f} \in Z_{f}, K_{t}^{f} \geq 0$ and $K_{t}^{f}=0 \forall t \notin\left[t_{0}^{f}, t_{1}^{f}\right]$ : ass. 3.a-c hold.

Def. 8 yields then a correct version of $H$; and the last point (which implies ass. 3.d) follows because, $\forall t, K \mapsto K_{t_{-}}$and $K \mapsto K_{t_{+}}$are continuous linear functions on $P$ : they exist, since $K \in P$ has locally bounded variation, and are then continuous since $\sup _{[-N, N]}\left|K_{t}\right| \leq$ $p_{N}(K)$. And ass. 1 is by ass. $4, k$ being bounded [11, thm. 8.14.14 p. 570].

Proposition 3. $Z=\{(H, K,-I) \in P \mid K \geq 0\}$. More precisely, $\forall(H, K,-I) \in P, K \geq$ $0, \exists\left(H^{f}, K^{f},-I^{f}\right) \in Z_{f}$ with $(H, K,-I)$ as Bochner integral s.t. $I_{t}^{f}$ is measurable for the product of $\mathcal{F}$ with the Lebesgue-measurable sets and s.t. $\forall t I_{t} \in L_{1}(\mu)$ and $\int I_{t}^{f} \mu(d f)=I_{t}$.

Further, the $\left(H^{f}, K^{f}\right)$ can all be chosen absolutely continuous w.r.t. $(H, K)$, with nonnegative densities, except for possible additional jumps in a fixed locally finite set $D$.

Proof. Inclusion $\subseteq$ is by [11, thm. 8.14.14 p. 570].

Let first $D_{0}=\left\{t \mid \mu\left(G_{t_{+}} \cap G_{t_{-}}\right)=0\right\} . \quad D_{0}$ is locally finite by ass. 2.c, thus so is $D_{1}=D_{0} \cup \mathbb{Z}$. Let $O_{n}$ enumerate the bounded, open intervals of $\complement D_{1}$. By ass. 2.c, at the boundary points $a, b$ of $O_{n}$ there exists $\varepsilon>0$ s.t. a positive mass of firms are alive on $[a, a+\varepsilon]$, similarly for $[b-\varepsilon, b]$. And by definition of $D_{0}$, at any point $t$ of $O_{n}$ there exists $\varepsilon_{t}>0$ s.t. a positive mass of firms are alive on $\left[t-\varepsilon_{t}, t+\varepsilon_{t}\right]$. Thus, by compactness of $[a, b]$, there exists $\varepsilon_{n}>0$ s.t. a positive mass of firms are alive on every subinterval of $[a, b]$ of length $\varepsilon_{n}$. Let now $k_{n}=\left\lceil\ln _{2}\left(\varepsilon_{n}^{-1}\right)\right\rceil$, and $D=D_{1} \cup \bigcup_{n}\left\{i 2^{-k_{n}} \in O_{n} \mid i \in \mathbb{Z}\right\}$. D is still locally finite, and for any 2 successive points $a, b \in D, \mu\left\{f \mid t_{0}^{f} \leq a, t_{1}^{f} \geq b\right\}>0$.

Let now $T(f)=\min \left\{t \in D \mid t \geq t_{0}^{f}\right\}, S(f)=\max \left\{t \in D \mid t \leq t_{1}^{f}\right\}, F_{0}=\left\{f \mid T_{f}<\right.$ $\left.S_{f}\right\} \in \mathcal{F}$. Thus, on $F_{0}, t_{0}^{f} \leq T_{f}<S_{f} \leq t_{1}^{f}$; so if we replace $F$ by $F_{0}, t_{0}^{f}$ by $T_{f}$ and $t_{1}^{f}$ by $S_{f}$, the new model still satisfies ass. 2 - point c because for any 2 successive points $a, b \in D, t_{0}^{f} \leq a \Rightarrow T_{f} \leq a$ and $t_{1}^{f} \geq b \Rightarrow S_{f} \geq b$, so $\mu\left\{f \mid T_{f} \leq a, S_{f} \geq b\right\}>0$. Now $t_{0}^{f}$ and $t_{1}^{f}$ take values in the locally finite set $D$, and clearly a solution $\left(H^{f}, K^{f},-I^{f}\right)$ for the new model will also be one for the original model when setting $\left(H^{f}, K^{f},-I^{f}\right)=0$ on $\complement F_{0}$.

Thus we may assume in addition that $t_{0}^{f}$ and $t_{1}^{f}$ take values in a locally finite set $D$.

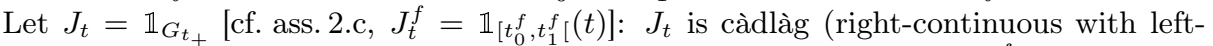
hand limits) and has locally integrable variation by ass. 2.b since $\forall f, V\left(J^{f}\right) \leq 2$. So, by the dominated convergence theorem and ass. 2.b, $m_{t}=\mu\left(G_{t_{+}}\right)=\int J_{t}^{f} \mu(d f)$ is càdlàg too, and clearly has locally bounded variation. Since $m_{t}$ is locally bounded away from 0 by ass. 2.c, $1 / m_{t}$ has locally bounded variation too, and so does $K_{t}$ by definition. Thus $K_{t}^{f}=$ $K_{t} J_{t}^{f} / m_{t}$ has locally integrable variation too (decompose locally $K_{t} / m_{t}$ as a difference of 2 positive increasing functions, and $J_{t}^{f}$ as a difference of 2 positive, increasing and integrable processes). Thus each $K^{f} \in Z_{f}$, having compact support. Further $m_{t}$ and $J_{t}$ are pure jump processes, with jumps only in the locally finite set $D$. Add to $D$ the countable set of jumps of the function $K_{t}$ : now all discontinuities of $K_{t}^{f}$ are in the countable set $D$, and the continuous part is absolutely continuous w.r.t. the continuous part of the function $K_{t}$. Thus $K_{t}^{f}$ is separably-valued, by lemma 20: by lemma $21, K^{f}$ is indeed Bochner-integrable.

Let similarly $I_{t}^{f}=I_{t} J_{t}^{f} / m_{t}$. Local joint integrability follows because $m_{t}$ is locally bounded away from 0 , and the rest is then obvious.

B.4. Transactions-based evaluation of profits. Profits equal proceeds from sales (the sum of capital sales $d H_{t}$ multiplied by their prices $p_{t}$ ), minus expenditure on investment plus rent of capital: $\int p_{t} d H_{t}-\int p_{t}^{I} I_{t} d t+\int r_{t} K_{t} d t$.

Comment 37. Here the integral w.r.t. $d H_{t}$ is really an integral w.r.t. a measure: even if at some $t, H_{t_{-}}, H_{t}$ and $H_{t_{+}}$are all different (say $H_{t}-H_{t_{-}}$represents the sales at time $t$ whose object is, on the transaction date, already registered in the name of the buyer, and $H_{t_{+}}-H_{t}$ those where it was still registered in the name of the seller), all those transactions occured on date $t$ (hence valued at the single price $p_{t}$ ) - i.e., the mass of $d H_{t}$ at $t$ equals $H_{t_{+}}-H_{t_{-}}$.

The previous formula, with its integrals over $\mathbb{R}$, is for a production plan with bounded support, say $\in Z_{f}$. To compute the profits on a bounded interval $J$ of an ongoing production plan, say in $Z$, one must enter the thought experiment that right before the beginning of $J$, say $a$, and right after the end, say $b$, available capital is sold and then re-bought, and thus subtract and add to the above the value of capital at those 2 moments. Thus, $p_{a_{-}} K_{a_{-}}$if $\{a\}$ is included in the interval, and $p_{a_{+}} K_{a_{+}}$else, and similarly at $b$. We obtain 
thus for profits on $J$, if $J=[a, b], p_{b_{+}} K_{b_{+}}-p_{a_{-}} K_{a_{-}}+\int_{J} p_{t} d H_{t}-\int_{J} p_{t}^{I} I_{t} d t+\int_{J} r_{t} K_{t} d t$, and if $J=] a, b\left[\right.$, replace there $a_{-}$by $a_{+}$and $b_{+}$by $b_{-}$, and similarly for $[a, b[$ and $] a, b]$. Here $\int_{J}$ has the usual meaning in the sense of the above defined measures.

Definition 10. For a bounded interval $J$ with endpoints $a \leq b$, say $J=[a, b]$, let $\pi_{J}^{0}(K, I)$ be the expression $p_{b_{+}} K_{b_{+}}-p_{a_{-}} K_{a_{-}}+\int_{J} p_{t} d H_{t}+\int_{J}\left(r_{t} K_{t}-p_{t}^{I} I_{t}\right) d t$. If $a$ (resp., $b$ ) is excluded from $J$, replace $a_{-}$above by $a_{+}$(resp. $b_{+}$by $b_{-}$), and, for $] a, b[$, require $a<b$.

Profits on $J$ are $\pi_{J}^{0}(K, I)$ if $H_{t}$ has locally bounded variation, both integrals and their sum are well-defined, and either $p_{b_{+}}$(resp., $p_{a_{-}}$) exists in $\mathbb{R}$ or $K_{b_{+}}$(resp., $K_{a_{-}}$) is 0 .

Lemma 22. Assume (a) $H_{t}^{f}$ has locally integrable variation, (b) $p_{t}$ and $r_{t} K_{t}^{f}-p_{t}^{I} I_{t}^{f}$ are jointly quasi-integrable on $J$, for $\mu(d f) \otimes d H_{t}^{f}$ and $\mu(d f) \otimes \lambda(d t)$ resp., and the sum of their integrals is well-defined, (c) $K_{b_{+}}^{f}$ and $K_{a_{-}}^{f}$ are the limit in $L_{1}$ of $K_{b+\varepsilon}^{f}$ and $K_{a-\varepsilon}^{f}$ when $\varepsilon \searrow 0,(d)$ either $p_{b_{+}}\left(\right.$resp., $\left.p_{a_{-}}\right)$exists in $\mathbb{R}$ or $K_{b_{+}}^{f}\left(\right.$ resp., $\left.K_{a_{-}}^{f}\right)$ is zero a.e.- - and similarly for non-closed bounded intervals $J$. Then $\pi_{J}^{0}$ aggregates well over $\left(K^{f}, I^{f}\right)$.

Remark 38. The integrability w.r.t. the measure $\mu(d f) \otimes d H_{t}^{f}$ on the product, rather than w.r.t. its marginal on $t$, or even w.r.t. the cumulative $t \mapsto \int V_{0, t}(H(f, \cdot)) \mu(d f)$, is essential. When $H^{f}$ is Bochner integrable, the latter does suffice. Cf. also cor. 19 below.

Proof. Use Fubini's theorem.

Next result relates aggregate profits over intervals to lifetime profits of firms - the aggregation of the plan which is $\left(H^{\prime}, K^{\prime},-I^{\prime}\right)$ on $F_{0}$ and 0 elsewhere is trivial.

Corollary 19. Assume $(H, K,-I) \in Z$, and profits $\pi_{J}^{0}(K, I)$ are well defined and $>0$. Then $\exists\left(H^{\prime}, K^{\prime},-I^{\prime}\right) \in \cap_{f \in F_{0}} Z^{f}$ with $\mu\left(F_{0}\right)>0$ and $\pi_{\mathbb{R}}^{0}\left(K^{\prime}, I^{\prime}\right)$ well defined and $>0$.

Proof. We first increase $J$ a bit, to make sure $K_{t}=I_{t}=0$ outside of $J$. First set $I$ to 0 outside of $J$. Let $a, b$ be the endpoints of $J$. If $b \notin J$ or $K_{b_{+}}=0$, set $K_{t}=0$ for $t \geq b$, and else choose $\varepsilon>0$ s.t. $p_{b+\varepsilon} \sim p_{b_{+}}$, and let $K_{t}=e^{-R(t-b)} K_{b_{+}}$for $\left.t \in\right] b, b+\varepsilon[$, $=0$ for $t \geq b+\varepsilon$ : computing now profits on $\left.\left.J^{\prime}=J \cup\right] b, b+\varepsilon\right]$ adds to profits the quantity $K_{b_{+}}\left(p_{b+\varepsilon} e^{-R \varepsilon}-p_{b_{+}}\right.$, which is arbitrarily small with $\varepsilon$, plus the non-negative quantity $\int_{b}^{b+\varepsilon}$ : for $\varepsilon$ sufficiently small, the new profits are still $>0$. Do the same at $a$. Now $K_{t}=I_{t}=0$ outside of (the new) $J$ - so any larger $J$ can as well be used. In particular, take its closure.

Construct now $\left(H^{f}, K^{f},-I^{f}\right) \in Z_{f}$ as in prop. 3, incuding the 'further' clause. Then the assumptions of lemma 22 are satisfied: $H_{t}^{f}$ has locally integrable variation by prop. 2 and lemma $18 ; p_{t}$ is $\mu(d f) \otimes d H_{t}^{f}$-integrable by the further clause, since the locally finite set $D$ intersects the bounded interval $J$ in a finite set, and any function is integrable for a distribution with finite support; the limits like $K_{b_{+}}^{f}$ are trivial and 0 . Finally, the quasiintegrability follows too, since $K^{f}$ and $I^{f}$ are piecewise proportional (with pieces which are products) to $K$ and $I$ resp., with the same non-negative proportionality factor. Thus profits are well defined, and aggregate to the positive aggregate profits. Finally, this piecewise aspect implies that $f \mapsto\left(K^{f}, I^{f}\right)$ is a simple function, so one the values that it takes (on a set of positive measure) must yield positive profits too. Let $\left(H^{\prime}, K^{\prime},-I^{\prime}\right)$ be that value.

Observe that this approach is one of "transactions-based" accounting: it is the cash-flow stemming from transactions that is recorded when they occur, and summed.

But the above formulae are pretty cumbersome, and make no sense when $K_{t}^{f}$ has unbounded variation. This is addressed next.

B.5. Integration by parts. Assume we know now further (e.g., by lemma 7) that $p_{t}$ has locally bounded variation. Then we want to use integration by parts in the previous formula. To this effect, define the linear functional $f_{a}^{b} K_{t} d p_{t}$ as, for $a<b$, $\left(p_{a_{+}}-p_{a}\right) K_{a_{+}}+\left(p_{b}-p_{b_{-}}\right) K_{b_{-}}+\int_{] a, b[} K_{t} d p_{t}$, where, at a jump $t$ of $p_{t}$ inside $] a, b[$, the contribution of the jump is counted as $\left(p_{t}-p_{t_{-}}\right) K_{t_{-}}+\left(p_{t_{+}}-p_{t}\right) K_{t_{+}}$. Then, $\forall a, b, c$, $f_{a}^{b}+f_{b}^{c}+f_{c}^{a}=0$ and $f_{a}^{b} f(G(x)) d F(G(x))=f_{G(a)}^{G(b)} f(x) d F(x) \forall G$ continuous and monotone (weakly increasing or decreasing) - using those formulae to define $f_{a}^{b}$ for $a \geq b$. 
With $f_{a}^{b}+f_{b}^{c}+f_{c}^{a}=0$, the above is the right concept to define a primitive $F$, such as to have $f_{a}^{b}=F(b)-F(a) .{ }^{36}$ To define the corresponding concept of integral over a bounded interval $J$, denoted $f_{J}$, we want (cf. lemma 24) continuity w.r.t. monotone sequences of $J$, and additivity (whenever the union of 2 disjoint intervals is an interval). Thus $f_{] a, b[}$ has the usual meaning, except for the treatment of atoms inside $] a, b\left[\right.$, and thus amounts to $F\left(b_{-}\right)-$ $F\left(a_{+}\right)$. Let also $f_{[t, t]} K_{t} d p_{t}=\left(p_{t}-p_{t_{-}}\right) K_{t_{-}}+\left(p_{t_{+}}-p_{t}\right) K_{t_{+}}$, just like for atoms inside $] a, b[$ - i.e., $F\left(t_{+}\right)-F\left(t_{-}\right)-$, and let $f_{] a, b]}=f_{] a, b[}+f_{[b, b]}, f_{[a, b[}=f_{[a, a]}+f_{] a, b[}$, and $f_{[a, b]}=$ $f_{[a, a]}+f_{] a, b[}+f_{[b, b]} \cdot{ }^{37}$ Then the integration by parts formula becomes: $\int_{[a, b]} p_{t} d K_{t}=$ $p_{b_{+}} K_{b_{+}}-p_{a_{-}} K_{a_{-}}-f_{[a, b]} K_{t} d p_{t}, \int_{] a, b]} p_{t} d K_{t}=p_{b_{+}} K_{b_{+}}-p_{a_{+}} K_{a_{+}}-f_{] a, b]} K_{t} d p_{t}$, etc. $^{38}$

\section{B.6. Marking to market.}

Definition 11. Let $p_{t}$ have locally bounded variation, and $K_{t}$ be locally integrable for the continuous part of $\left|d p_{t}\right|+\lambda(d t)$, and s.t. $p_{t} \neq p_{t_{+}} \Rightarrow K_{t_{+}}$exists and $p_{t_{-}} \neq p_{t} \Rightarrow K_{t_{-}}$ exists, and s.t. $\sum_{t \in J}\left|p_{t_{+}}-p_{t}\right| K_{t_{+}}+\left|p_{t}-p_{t_{-}}\right| K_{t_{-}}<\infty$. If $\int_{J}\left(r_{t} K_{t}-p_{t}^{I} I_{t}\right) d t$ is well-defined, profits on a bounded interval $J, \pi_{J}(K, I) \stackrel{\text { def }}{=} f_{J} K_{t} d p_{t}+\int_{J}\left[\left(r_{t}-\delta p_{t}\right) K_{t}+\left(p_{t}-p_{t}^{I}\right) I_{t}\right] d t$.

In particular, any function $K_{t}$ that has left- and right hand limits at every point satisfies this for any $p$ with locally bounded variation.

Lemma 23. $\pi_{J}(K, I)=\pi_{J}^{0}(K, I)$ whenever both are well-defined, i.e., whenever $p$ and $K$ both have locally bounded variation, and $\int_{J}\left(r_{t} K_{t}-p_{t}^{I} I_{t}\right) d t$ is well-defined.

Proof. Use first lemma 18 and def. 8, and the fact that $p_{t}$ is a fortiori Lebesgue measurable and locally bounded, to re-write $\pi_{J}^{0}(K, I)$ as $p_{b_{+}} K_{b_{+}}-p_{a_{-}} K_{a_{-}}-\int_{J} p_{t} d K_{t}+\int_{J}\left[\left(r_{t}-\delta p_{t}\right) K_{t}+\right.$ $\left.\left(p_{t}-p_{t}^{I}\right) I_{t}\right] d t$. Then use the above integration by parts formula.

Next lemma localises profits precisely in time:

Lemma 24. If the conditions of def. 11 hold for all bounded intervals $J, \pi_{J}(K, I)$ is well-defined for all bounded Borel sets $J$, and is a countably additive on them.

\footnotetext{
${ }^{36} F$ has locally bounded variation: decompose both $p_{t}$ and $K_{t}$ as differences of 2 positive increasing functions; by bilinearity one can assume both are such. Clearly then $F$ is increasing too.

${ }^{37}$ One can view $f$ as a usual integral: view the linear functional $f$ as linear map from functions $p_{t}$ of locally bounded variation to Radon measures $d p_{t}$ on the space $\mathbb{T}_{\tau}=\{t \in \mathbb{R}\} \cup\left\{t_{-} \mid t \in\right.$ $\mathbb{R}\} \cup\left\{t_{+} \mid t \in \mathbb{R}\right\}, \mathbb{T}_{\tau}$ being ordered in the obvious way $\left(t_{-}<t<t_{+}\right)$, and endowed with the order topology (having the open intervals as basis). $\mathbb{T}_{\tau}$ is locally compact, by standard arguments, and each singleton $\{t\}$ is open, and has measure 0 under $d p_{t}$, so the measure has its support in the subspace $\mathbb{T}_{\delta}$ ( $\delta$ for $2, \tau$ for 3 ) consisting only of the points $t_{-}$and $t_{+}$. $\mathbb{T}_{\delta}$ is locally compact, totally disconnected, and separable, but still the Borel $\sigma$-field (equals the Baire $\sigma$-field, or that spanned by the intervals, or the inverse images of Borel sets of $\mathbb{R}$ modulo a countable set) is not separable. $\mathbb{T}_{\delta}$ is the Stone-Čech space of the quotient (e.g., identified with the space of càdlàg functions) of the space bounded functions on $\mathbb{R}$ converging to 0 at $\infty$ and having everywhere leftand right-hand limits (i.e., the Banach space (in the sup-norm) spanned by the indicator functions of bounded intervals), by the subspace $c_{0}(\mathbb{R})$ of functions whose left- and right-hand limits are 0 .

The conditions on $K_{t}$ in def. 11 are then exactly those needed for a function $K$ on $\mathbb{R}$ to extend unambiguously to an equivalence class in $L_{1}^{\text {loc }}$ for the Radon measure $d p_{t}$ on $\mathbb{T}_{\delta}$; and intervals $J$ in $\mathbb{R}$ are mapped to their inverse images in $\mathbb{T}_{\delta}$. Everything extends thus to $d p_{t}$-measurable sets $J$.

${ }^{38}$ Indeed, by linearity, suffices first to consider the case where $K_{t}$ and $p_{t}$ are increasing, next one can decompose both into their continuous part and a sum of jumps part, next, neglecting the tail of the latter, one is reduced to the case where each of $K_{t}$ and $p_{t}$ is either continuous or a single jump $\mathbb{1}_{t \geq t_{0}}$ or $\mathbb{1}_{t>t_{0}}$. Further, since the value of $K_{t}$ at points of discontinuity plays no rôle in the formula, we can assume $K_{t}$ right-continuous, so the integral w.r.t. $d K_{t}$ is a plain Stieltjes integral. Thus, if $p_{t}$ is continuous, all integrals involved are Stieltjes integrals, and the formula is classical for this case. Similarly if $K_{t}$ is continuous, since then $f_{[t, t]} K_{t} d p_{t}=\left(p_{t_{+}}-p_{t_{-}}\right) K_{t}$, so the integral is the Stieltjes integral that w.r.t. the right-continuous version of $p_{t}-$ and replacing $p_{t}$ by this version does not change the other integral, by continuity of $K_{t}$. Remain thus the cases where $p_{t}=\mathbb{1}_{t \geq t_{0}}$ or $p_{t}=\mathbb{1}_{t>t_{0}}$, and $K_{t}=\mathbb{1}_{t \geq t_{1}}$ (by right-continuity), with $t_{i} \in[a, b]$. If $t_{1} \neq t_{0}$ the formula results again from the classical formula for Stieltjes integrals, and for $t_{1}=t_{0}$ the formulae result from a straight computation (distinguishing the case where $t_{0}$ is a boundary point of $J$ from the others).
} 
Proof. Follows from fn. 37, which implies that $f_{B} K_{t} d p_{t}$ is a measure on the Borel sets of $\mathbb{T}_{\delta}$, and that those include the inverse images of all Borel sets of $\mathbb{R}$. The other integral in $\pi_{J}(K, I)$ is clearly countably additive w.r.t. $J$.

Lemma 25. Assume that $K_{t}^{f}$ is $\mu \otimes\left(\left|d p_{t}\right|+\lambda(d t)\right)$-measurable, that $\int K_{t}^{f} \mu(d f)$ is, locally in $t$, integrable under $\lambda$ and the continuous part of $\left|d p_{t}\right|$, that $p_{t} \neq p_{t_{+}} \Rightarrow \lim _{\varepsilon \searrow 0} K_{t+\varepsilon}^{f}=$ $K_{t_{+}}^{f}$ a.e. and in $L_{1}$ and $p_{t_{-}} \neq p_{t} \Rightarrow \lim _{\varepsilon \searrow 0} K_{t-\varepsilon}^{f}=K_{t_{-}}^{f}$ a.e. and in $L_{1}$, and that $\sum_{t \in J}\left|p_{t_{+}}-p_{t}\right| K_{t_{+}}^{f}+\left|p_{t}-p_{t_{-}}\right| K_{t_{-}}^{f}$ is integrable. If further $r_{t} K_{t}^{f}-p_{t}^{I} I_{t}^{f}$ is jointly quasiintegrable on $J$, then $\pi_{J}\left(K^{f}, I^{f}\right)$ is a.e. well-defined and aggregates well.

Proof. Profits are well-defined for a.e. firm by def. 11: local integrability of $K_{t}^{f}$ in $t$ follows from the second assumption and Fubini, and since the countable sum of values of capital is integrable in $f$, it is finite for a.e. $f$.

Profits for aggregate $K, I$ are well-defined also by def. 11, since the convergence of $K_{t}^{f}$ at points of price discontinuities are in $L_{1}$.

The profits aggregate well: the order of integration in the last integral over $J$ in the formula for profits in def. 11 is interchangeable by the joint quasi-integrability of $r_{t} K_{t}^{f}-p_{t}^{I} I_{t}^{f}$ and boundedness of $p_{t}, J$, and local integrability of $K_{t}^{f}$ and $I_{t}^{f}$. The first integral in that formula can be decomposed, by its definition, into an integral of the discontinuous and a continiuous part of $p$. The latter is a regular integral and so joint integrability of $K_{t}^{f}$ allows the use of Fubini. For the first term, it is sufficient to show that $\int K_{t_{+}}^{f} \mu(d f)=K_{t_{+}}$ and the same for $K_{t_{-}}$, but that follows from the convergence in $L_{1}$ of $K_{t+\varepsilon}^{f}$.

If $K$ has left- and right hand limits, the $L_{1}$ convergence conditions are equivalent to: for any monotone sequence $t_{n}, \lim _{n} \int K_{t_{n}}^{f} \mu(d f)=\int \lim _{n} K_{t_{n}}^{f} \mu(d f)$, or still equivalently, $K_{t}^{f}$ is locally uniformly integrable. ${ }^{39}$ For a feasible production plan, $K_{t}$ being continuous and $K_{t}^{f} \geq$ 0 , this is still equivalent to the more intuitive market clearing: $\int K_{t_{-}}^{f} \mu(d f)=\int K_{t}^{f} \mu(d f)=$ $\int K_{t_{+}}^{f} \mu(d f)$. All conditions are satisfied when $K$ has locally integrable variation.

This formula for profits is on the contrary a form of "marking to market" accounting: the integral $f_{J} K_{t} d p_{t}$ shows that profits and losses are added daily to the account by adding to past profits the impact of today's price-variation on the value of the assets. Transactions at arbitrage-free prices don't alter the value of the portfolio, so are immaterial in this system.

Comment 39. The more is known about $p$, the more plans can be evaluated this way. For example, if $p^{I}=p$ is locally Lipschitz and lemma 10.a4 holds, then any jointly integrable $K_{t}^{f}$ satisfies the assumptions of lemma 25 .

Because of this, there is no good reason to require anything more of $K$ in the model than local joint integrability; as a consequence however, this implies that as long as the Lipschitz character of $p$ is not proved, the only arbitrage arguments we can use are that production plans satisfying the stronger assumptions above, would not be profitable.

Comment 40. Even equilibrium prices can have discontinuities and singular parts (as described in thm. 1), so one really needs to allow for any $p_{t}$ of locally bounded variation-if it was continuous, standard integration theory would suffice for the integration by parts.

Comment 41. In applying the above in lemma 7 to obtain the zero-profit conditions on prices, we use for further strength only the most restrictive production plans, of lemma 22 .

\section{Appendix C. Analyticity properties of the BGe Graph}

Lemma 26. For $O \subseteq \mathbb{C} \times \mathbb{C}^{n}$ open and $F: O \rightarrow \mathbb{C}$ analytic, $\frac{F(x, z)-F(y, z)}{x-y}$ is so too on $\{(x, y, z) \mid(x, z) \in O,(y, z) \in O\}$.

Proof. Suffices to prove analyticity at points of the form $\left(x_{0}, x_{0}, z_{0}\right)$. Replace $F$ by its power series around $\left(x_{0}, z_{0}\right) \in O$, getting $a_{n}(z) \frac{\left(x-x_{0}\right)^{n}-\left(y-x_{0}\right)^{n}}{\left(x-x_{0}\right)-\left(y-x_{0}\right)}$ as a typical term, and then verify that after division the resulting power series still has positive (e.g., the same) radius of convergence.

${ }^{39}$ Clearly, ass. 3.d is a weakening of this local uniform integrability. 
Lemma 27. For a bounded measure $h$ on $[0,1]$ let $X_{h}=\digamma(\mathfrak{r}) \int e^{s \mathfrak{r}} h(d s)$, and $\mathcal{H}_{h}: \mathbb{C}^{4} \rightarrow$ $\mathbb{C}:(\mathfrak{r}, R, \sigma, \eta) \mapsto \frac{\int h(d s)-X_{h}}{\mathfrak{r}} .\left(\mathcal{H}_{h}(\mathfrak{r})=\frac{\tilde{H}_{h}(\mathfrak{r})}{\mathfrak{r}}\right) . \mathcal{H}_{h}$ is analytic except for poles at $(1-\sigma) \mathfrak{r}-$ $\eta=2 n \pi \mathrm{i}$ with $n \neq 0$.

Proof. $X_{h}$ is analytic except for those poles: for $\digamma(\mathfrak{r})$ notice that $\Phi(z)$ is entire by lemma 26 and has as only zeros $2 n \pi \mathrm{i}$ with $n \neq 0$. And $\int e^{s \mathfrak{r}} h(d s)$ is entire because $h$ has compact support. Remains thus to prove that $\mathcal{H}$ is analytic at any point with $\mathfrak{r}=0 .{ }^{40}$ Since $\digamma(0)=1$ and $X_{h}$ at $\mathfrak{r}=0$ is just $\int h(d s)$, this follows by lemma 26 .

Appendix D. A lemma of B. Weiss

Next lemma is due to Weiss [B. 22].

Lemma 28. For $f, g \geq 0$ Lebesgue-measurable on $\mathbb{R}$, if $f$ is not locally integrable at $x$, $f \star g=\infty$ a.e. on $x+\{g>0\}$. So, if $f \star g<\infty$ a.e. and $g \neq 0, f$ is locally integrable.

Proof. Assume $f$ is not locally integrable, say at 0 ; then $\exists \varphi: 0 \leq \varphi \leq f$, with compact support, say $[0,1]$, and s.t. $\int_{\varepsilon}^{1} \varphi<\infty \forall \varepsilon>0$, and $\int_{0}^{1} \varphi=\infty$. Further $g$ can be minorised by a positive multiple of the indicator of a compact set $K$, which can be assumed to lie in an interval of length 1 , and shifted to $[-1,0]$.

With $f_{n}=\min \{\varphi, n\}$ and $I_{n}=\int f_{n}, h_{n}=f_{n} \star \mathbb{1}_{K}$ is carried by $[-1,1]$ and $\leq I_{n}$. Then $\left\|h_{n}\right\|_{\infty} \leq I_{n}$, thus $\lambda(K) I_{n}=\int h_{n} \leq 2 N+I_{n} \lambda\left\{x \mid h_{n} \geq N\right\} \forall N \geq 0$, so $\lambda\left\{x \mid h_{n} \geq N\right\} \geq$ $\lambda(K)-\frac{2 N}{I_{n}}$. Since $I_{n} \rightarrow \infty, 2 \geq \lambda\left\{x \mid h_{\infty} \geq N\right\} \geq \lambda(K)$, so $\lambda\left\{x \mid h_{\infty}=\infty\right\} \geq \lambda(\bar{K})$.

For $x \notin K, h_{\infty}(x)=\int_{x-K} \varphi<\infty$, since $x-K$ is a compact set disjoint from 0 , and $\varphi$ is locally integrable there. Thus $h_{\infty}=\infty$ a.e. on $K$. Since $\{g>0\}$ can be approximated from inside by finite unions of such sets $K$, the conclusion follows.

\section{REFERENCES}

[1] Aumann, R. J. (1965): "Integrals of set valued functions," Journal of Mathematical Analysis and Applications, 12, 1-12.

[2] Bommier, A., And R. D. Lee (2003): "Overlapping generations models with realistic demography," Journal of Population Economics, 16, 135-160.

[3] Cass, D., And M. E. YAari (1967): "Individual Saving, Aggregate Capital Accumulation, and Efficient Growth," in Essays on the Theory of Optimal Economic Growth, ed. by K. Shell, pp. 233-268. MIT Press, Cambridge (MA).

[4] Č́elidze, V. G., And A. G. DžvaršĚ̃̌šlli (1989): The theory of the Denjoy integral and some applications, vol. 3 of Series in Real Analysis. World Scientific, Original Russian edition published by Tblisi University Press, Tblisi, 1978.

[5] D'Albis, H., and E. Augeraud-Véron (2007): "Balanced cycles in an OLG model with a continuum of finitely-lived individuals," Economic Theory, 30, 181186.

[6] D'Albis, H., and E. Augeraud-VÉron (2009): "Competitive growth in a life-cycle model: existence and dynamics," International Economic Review, 50(2), $459-484$.

[7] Debreu, G. (1967): "Integration of correspondences," in Proceedings of the Fifth Berkeley Symposium on Mathematical Statistics and Probability, vol. II, pp. 351-372. University of California Press, part 1.

[8] Demichelis, S., and H. M. Polemarchakis (2007): "The determinacy of equilibrium in economies of overlapping generations," Economic Theory, 32(3), $3461-475$.

[9] Diamond, P. (1965): "National Debt in a Neoclassical Growth Model," American Economic Review, LV(5), 1126-1150, Part 1.

[10] Doob, J. L. (1953): Stochastic Processes. John Wiley \& Sons, Inc., New York.

[11] Edwards, R. E. (1965): Functional Analysis. Holt, Rinehart and Winston.

\footnotetext{
${ }^{40}$ For $h=\varphi$, this is the intersection of the BGE and GRE graphs, see e.g., sect. 5.3.
} 
[12] Gale, D. (1973): "Pure Exchange Equilibrium of Dynamic Economic Models," Journal of Economic Theory, 6, 12-36.

[13] GAN, Z., AND S.-H. P. LAU (2010): "Demographic structure and overlapping generations: A simpler proof with more general conditions," Journal of Mathematical Economics, 46, 311-319.

[14] Kehoe, T. J., And D. K. Levine (1984): "Regularity in overlapping generations exchange economies," Journal of Mathematical Economics, 13(1), 69-93.

[15] (1985): "Comparative Statics and Perfect Foresight in Infinite Horizon Economies," Econometrica, 53(2), 433-453.

[16] Mertens, J.-F., And A. Rubinchik (2010): "Welfare evaluation of policies in an overlapping generations growth model," Working Paper.

[17] - (2011a): "Pareto Optimality of the Golden Rule equilibrium in Overlapping Generations Model with Production and Transfers," Working Paper.

[18] — (2011b): "Regularity and stability of equilibria in an overlapping generations growth model," Discussion paper, http://econ.haifa.ac.il/ arubinchik/papers/Reg_Stab_OLG.pdf.

[19] (2011c): "Separate control over the local and the asymptotic behaviour in $L_{p}$ spaces," Discussion Paper 2011/1, University of Haifa, http: //econ.haifa.ac.il/ arubinchik/papers/Lpq_paper.pdf.

[20] (2012): "Intergenerational equity and the discount rate for policy analysis," Macroeconomic Dynamics, 16, 61-93, http://journals.cambridge. org/repo_A84Ar9FF.

[21] Samuelson, P. A. (1958): "An Exact Consumption-Loan Model of Interest with or without the Social Contrivance of Money," The Journal of Political Economy, 66(6), 467-482.

[22] WeISS, B. (2010): personal communication.

[23] YaAri, M. E. (1964): "On the Consumer's Lifetime Allocation Process," International Economic Review, 5(3), 304-317. 